\title{
Variable Feedback Latency Compensation for the LMS- Based Smart Antenna Receiver
}

\author{
by \\ Yin Fung Tang \\ B.A.Sc. University of British Columbia, Vancouver, B.C. Canada, 2000
}

\begin{abstract}
A Thesis Submitted to the
Faculty of Graduate Studies and Research

In partial fulfillment of the Requirements for the degree of

Master of Applied Science

Ottawa-Carleton Institute for Electrical and Computer Engineering Department of Electronics

Faculty of Engineering

Carleton University

Ottawa, Ontario

May 2005
\end{abstract}

Yin Fung Tang, 2005 
Library and

Archives Canada

Published Heritage

Branch

395 Wellington Street

Ottawa ONK1A ON4

Canada
Bibliothèque et

Archives Canada

Direction du

Patrimoine de l'édition

395 , rue Wellington

Ottawa ON K1A 0N4

Canada
Your file Votre référence
ISBN:
Ourfile Notre reference
ISBN:

\section{NOTICE:}

The author has granted a nonexclusive license allowing Library and Archives Canada to reproduce, publish, archive, preserve, conserve, communicate to the public by telecommunication or on the Internet, loan, distribute and sell theses worldwide, for commercial or noncommercial purposes, in microform, paper, electronic and/or any other formats.

The author retains copyright ownership and moral rights in this thesis. Neither the thesis nor substantial extracts from it may be printed or otherwise reproduced without the author's permission.
AVIS:

L'auteur a accordé une licence non exclusive permettant à la Bibliothèque et Archives Canada de reproduire, publier, archiver, sauvegarder, conserver, transmettre au public par télécommunication ou par l'Internet, prêter, distribuer et vendre des thèses partout dans le monde, à des fins commerciales ou autres, sur support microforme, papier, électronique et/ou autres formats.

L'auteur conserve la propriété du droit d'auteur et des droits moraux qui protège cette thèse. $\mathrm{Ni}$ la thèse ni des extraits substantiels de celle-ci ne doivent être imprimés ou autrement reproduits sans son autorisation.
In compliance with the Canadian

Privacy Act some supporting forms may have been removed from this thesis.

While these forms may be included in the document page count, their removal does not represent any loss of content from the thesis.
Conformément à la loi canadienne sur la protection de la vie privée, quelques formulaires secondaires ont été enlevés de cette thèse.

Bien que ces formulaires aient inclus dans la pagination, il n'y aura aucun contenu manquant.

\section{Canada}




\section{Abstract}

The combined beam-former and equalizer architecture uses the error signal produced by the equalizer to update the tap weights for both the beam-former and the equalizer. This thesis has shown that the latency of the error signal going to the beamformer depends on the tap weights of the equalizer, which in turn depend on the latency of the channel. It is shown that if the error signal latency is not adjusted properly, it will ultimately affect the performance of the receiver system. This thesis proposes a system that can be used to compensate for the variable error signal feedback latency. In the proposed system, extra delay is added dynamically in the update engine in order for the beam-former to compensate for the variable amount of latency in the error signal. In simulation, the proposed system is able to improve the bit error rate (BER) performance of the receiver and the convergence time of the adaptive algorithm. In order to demonstrate the effectiveness of the system in a real-life situation. we have built a signal capture system. Using the captured signal, it is shown that the error signal latency compensation system improves the overall performance of the receiver. Based on the captured data, the best case improvement is over $1.5 \mathrm{dbm}$ of transmit power. 


\section{Table of Contents}

$\begin{array}{ll}\text { Abstract } & \text { ii }\end{array}$

Table of Contents iii

List of Figures $\quad$ vi

List of Tables $\quad \mathbf{x}$

Nomenclature $\quad$ xi

$\begin{array}{ll}\text { Acknowledgement } & \text { xiii }\end{array}$

1. Introduction 1

I.1. Motivation I

1.2. Thesis Objectives 3

1.3. Thesis Outline 4

2. A Smart Antenna Enabled Digital Transmission System Model 6

2.1. Transmitter Model 7

2.2. Channel Model 9

2.3. Receiver Model 12

2.4. Smart Antenna Signal Processor 16

$\begin{array}{ll}\text { 2.4.1. Beam-former Model } & 17\end{array}$

2.4.2. Matched Filter Model 19

2.4.3. Equalizer Model 20

2.5. Combined beam-former and equalizer $\quad 22$

2.5.1. Problems with the Traditional Beam-former 2.3

2.5.2. Combining the Beam-former and Equalizer 24

2.6. Discussion 26

3. Adaptive Beam-forming and Equalization Parameter Selection 27

3.l. Simulation Model 28

3.2. Evaluation Criteria 30

3.2.1. Bit Error Rate Perlormance $\quad 30$

3.2.2. Convergence Time 31

3.2.3. Computational Efficiency

3.3. Parameter Selections for the Combined beam-former and equalizer $\quad 34$

3.3.1. Beam-former Length

iii 
3.3.2. FSE Equalizer Length

3.3.3. Fractionally Spaced Forward Filter Step Size 40

3.3.4. Ideal Beam-former Parameters 43

3.4. Properties of the Combined beam-former and equalizer 45

3.4.1. Wider Acquisition Range 45

3.4.2. Combining Signals from Multiple Paths 47

3.5. Problems with Combined beam-former and equalizer 48

3.5.1. Sensitivity to the Magnitude of the Eirror Signal 49

3.5.2. Slow Convergence Time 49

3.5.3. Variable Error Signal Feedback Delay 50

3.6. Discussion and Conclusion 55

4. Variable Feedback Delay Beam-former Equalization 57

4.1. Systems to Equalize the Variable Error Feedback Delay 58

4.2. Modulate the Delay of the Desired Signal 60

4.3. Modulate the Delay of the Desired Signal Delay to the Beam-former 63

4.3.1. Desired Signal Delay Model I

4.3.2. Desired Signal Delay Model II 6.5

4.4. Modulate the Delay of the Error Signal to the Beam-former 68

4.5. Modulate the Loop Delay. Constant in the Beam-former 72

4.6. Design Comparison 74

5. Signal Capture System $\quad 76$

5.1. Capture System 76

5.1.1. Antennas

5.1.2. RF Fromt-end Assemblies 78

$\begin{array}{lll}\text { 5.1.3. A/D Capture Board } & 79\end{array}$

5.1.4. Carrier Recovery System 79

5.2. Pilor Signal Modulation 80

5.3. Adaptive Algorithms. 83

$\begin{array}{ll}\text { 5.3.1. Combined LMS and CMA Algorithm } & 83 \\ \text { 5.3.2. } & 83 \text { ald }\end{array}$

5.3.2. Spread Pilot Signal LMS Algorithm 84

5.4. Environment Setup 85

5.4.1. Multi-antenna Emulation 86

5.4.2. Multi-path Emulation $\quad 87$

6. Experimental Results $\quad 90$

6.1. Experiment Setup 90

6.1.1. Fxperiment Test Environment 90

6.1.2. Experiment Scenarios 91

6.2. Performance Comparison 93

6.2.1. The Combined beam-former and equalizer System

6.2.2. Variable Delay Error Signal Feedback Compensation System 99

$\begin{array}{ll}\text { 6.3. Discussion } & 105\end{array}$ 
6.3.1. The Signal Capture System 10.5

6.3.2. The Combined beam-former and equalizer System 106

6.3.3. The Variable Delay Error Signal Feedback Compensation System 106

7. Conclusion and Future Work 109

7.1. Conclusions: 109

$\begin{array}{ll}\text { 7.2. Future Work } & 112\end{array}$

A. Appendix - Carrier Recovery System 114

A.1. Problem of Using a Digital Costas Loop to Recover the Carrier $\quad 114$

A.2. Solutions for the Current System 116

A.3. Solutions for Future Systems $\quad 118$

$\begin{array}{ll}\text { References } & 119\end{array}$ 


\section{List of Figures}

Figure 1. A smart antenna enabled digital transmission system model consists of four major components: the transmitter, the channel, the receiver/ sampler, and the smart antenna signal processor.

6

Figure 2. A simple transmitter consists of four basic building blocks: a symbol source, a symbol mapper, a pulse shape filter, and a modulator.

Figure 3. The transmitted signal travels $\mathrm{K}$ different paths to reach the receiver antennas; the attenuation of each path follows the Rayleigh distribution. 9

Figure 4. The diagram shows how simple trigonometry can be used to approximate the value of $d_{k, a}$ 11

Figure 5. The receiver front end demodulates the bandpass signal by multiplying it with a locally generated version of the carrier, and the resulting baseband signal is low-pass filtered to remove any high frequency noise, before it is sampled by the sampler.

Figure 6. A traditional beam-former uses the immediate output of the beam-former to generate the necessary error signal. 17

Figure 7. An adaptive digital beam-former consists of a weighted sum network to generate a single stream of sampled data. 18

Figure 8. The structure of an adaptive equalizer consists of two equalizers: the feedforward filter, and the feedback filter. Both equalizers are adaptive and their tap weights are updated using the same error signal 21

Figure 9. For the combined beam-former and equalizer architecture, the error signal for both the beam-former and equalizer is produced by comparing the output of the equalizer against the desired signal 25

Figure 10. There are three different parameters that can be adjusted in the combined beam-former and equalizer architecture: the length of the beam-former, the downsampler ratio, and finally, the length of the feedforward filter. 34

Figure 11. As the value of $L_{k i}$ varies, the MSE declines much more quickly; however, the improvement diminishes as $L_{k t}$ becomes larger. 36

Figure 12. The graph shows the MSE signal going into the beam-former for a different value of $L_{t r}$ 39

Figure 13. This simulation is run with 2 interference sources. Although none of the systems seems to be able to converge to a solution, the systems with a higher value of $L_{k r}$ seem to perform slightly better than systems with a lower value of $L_{r i} \ldots \ldots .40$ 
Figure 14. Based on the plot above, one can conclude that the system with the larger value of $L_{k \%}$ tends to converge faster and the magnitude of the steady-state MSE signal is lower.

Figure 15. The graph shows the magnitude MSE signal of the receivers with different down sampling ratio, $\chi$. As the ratio decreases, there is marginal improve for the convergence performance.

Figure 16. The graph shows the magnitude of the MSE signal of the receivers with the down-sampling ratio, $\chi$, set to 8 . Unlike the graph shown previously, the delay of the training has been tuned; as a result, the system is able to converge. Further tuning can improve the converge speed.

Figure 17. The graph shows the MSE signal going into the two different beam-formers. The combined beam-former and equalizer architecture is able to re-adapt to the loss of the line of sight, while the traditional beam-former architecture fails to compensate.

Figure 18. The diagram above shows the magnitude of the taps of the equalizer after the receiver system has entered a steady state. 48

Figure 19. By comparing the magnitude of MSE over time, it is shown from the above graph that the convergence time for the traditional LMS beam-former is faster than that of the combined beam-former and equalizer

Figure 20. This is a simplified version of the digital transmission system, with only a simple delay channel, a single FIR filter beam-former, and a simple equalizer...... 51

Figure 21. This graph shows the BER of the system as the error signal feedback delay varies. 55

Figure 22. The desired signal is delayed such that it shifts the peak of the tap values to the center; hence, the error signal loop delay becomes constant. The delay processor measures the delay introduced by the feedforward filter and adjusts the variable delay element to compensate.

Figure 23. The graph compares the BER of the combined beam-former and equalizer with the desired signal delay being modulated against the traditional beam-former design.

Figure 24. The delay processor calculates the proper delay value and programs the variable delay element such that the desired signal matches up with the incoming signal to the beam-former.

Figure 25. The secondary equalizer uses signals from one of the antennas as input. Although the signal is not enhanced by the beam-former, the equalizer is still able to pick up the desired signal in the face of severe interference.

Figure 26. The graph compares the BER of the combined beam-former and equalizer with the desired signal delay being modulated against the traditional beam-former design. 68 
Figure 27. By modulating the delay of the error feedback, the total loop delay could stay constant because the variable delay unit could compensate for any additional delay introduced by the FSE. 69

Figure 28. The graphs on the right are the BERs of the system that uses the error signal delay modulator, and the graphs on the left are the BERs of the original combined beam-former and equalizer system. 70

Figure 29. The graph shows the magnitude of the MSE signal for two random simulations. 71

Figure 30. The graph shows the BER of the loop delay constant modulation system against an ordinary combined beam-former and equalizer system. 73

Figure 31. The graph shows the BER of the loop delay constant modulation system against the system, which modulates the delay of the error signal to the beamformer. 74

Figure 32. The capture system consists of four major components: the antenna, the RF front-end assemblies, the A/D capture board, and the carrier recovery systems..... 77

Figure 33. The antennas used in the capture system are patch antennas, which radiates only in the forward direction with beam width of 170 degrees. 78

Figure 34. The pilot signal is embedded in the data signal to help the beam-former in the receiver to home in on the signal. The ratio of the pilot signal to the data signal will determine the speed of the algorithm convergence on the receiver side.

Figure 35. The diagram shows how the data symbols are interleaved with the pilot signal symbols. Instead of embedding the pilot signal symbols in each sub-frame, the pattern of the pilot signal symbols are repeated only during each super-frame. ..... 85

Figure 36. This diagram shows how the test should be conducted for multiple antenna emulation. In test session \#1, the signal going to the right hand side is captured by the receiver on the right. In test session \#2, the antenna array is moved to the left to capture the signal reflected to the left hand side.

Figure 37. The diagram shows how the test should be conducted for multi-path emulation. In test session \#1, a short delay spread channel is emulated. In the test session \#2, the environment is changed to create a different signal path. 89

Figure 38. The layout and the dimension of the room in which the experiments are conducted are shown in the above figure: in addition, the two capture environments are also presented. 91

Figure 39. Photo of the capture system. 92

Figure 40. This diagrams show the MSE magnitudes for all of the different receivers, when the transmit power varies from $-5 \mathrm{dBm}$ to $5 \mathrm{dBm}$ in a channel with little delay spread. 95

Figure 41. This diagrams show the MSE magnitudes for all of the different receivers when the transmit power varies from $-4 \mathrm{dBm}$ to $5 \mathrm{dBm}$ in a multi-path channel..... 97 
Figure 42. This diagram shows the average BER performance for a channel with little delay spread.

Figure 43. This diagram shows the average BER performance for a channel with a large delay spread

Figure 44 The diagrams show the MSE magnitudes for both the compensated and the uncompensated system when the transmit power varies from $-4 \mathrm{dBm}$ to $5 \mathrm{dBm}$ in a channel with little delay spread..

Figure 45. The diagrams show the MSE magnitude for both the compensated and the uncompensated system when the transmit power varies from $-4 \mathrm{dBm}$ to $5 \mathrm{dBm}$ in a large delay spread channel.

Figure 46. The average BER performance for a channel with little delay spread shows that the compensated system is only marginally better than the uncompensated... 103

Figure 47. The average BER performance for a channel with a large delay spread is slightly better for the compensated system than the uncompensated system. ........ 104

Figure 48. The diagram shows the output of the latency calculator and the cumulative BER over time 107

Figure 49. The diagram shows the structure of the Costas Loop for QAM-4 signals.... 115

Figure 50. An alternate implementation of the capture system uses a single PLL to generate the IF frequency to down-convert the signal from the antenna.... 118 


\section{List of Tables}

Table 1 The following table shows the value for each of the channel parameters... 28

Table 2 The following table shows the value for each of the transmission system parameters.................................................................................................. 29

Table 3 The following table shows the value for each of the system variables at

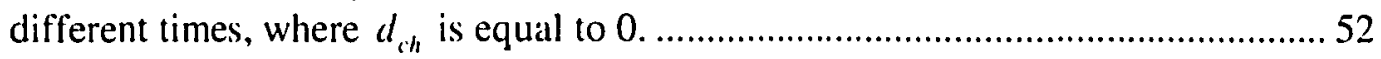

Table 4 The following table shows the values for each of the system variables at

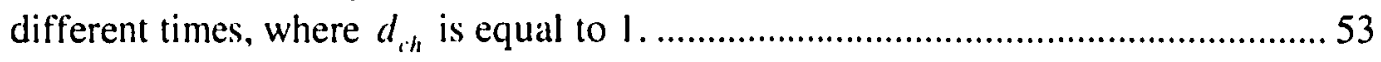

Table 5 The following table shows the value for each of the channel parameters.... 58

Table 6 The following table shows the value for each of the transmission system

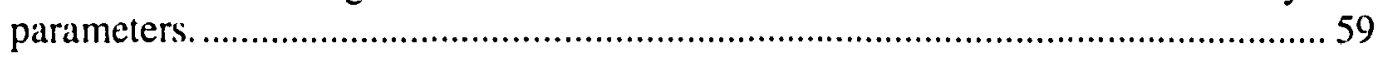

Table 7 The following table shows the value for each of the transmission system parameters ................................................................................................. 94

Table 8 The following table shows the value for each of the transmission system parameters. 100 


\section{Nomenclature}

Voltage Control Oscillator

Radio Frequency

Intermediate Frequency

Least Mean Square

MUltiple SIgnal Classification

Constant Modulus Algorithm

Recursive Least Squares

Analog to Digital Converter

Digital Signal Processor

Digital Adaptive Beam-Former

Direction Of Arrival

Quadrature Amplitude Modulation

Quadrature Phase Shift Key

Angle of Arrival
VCO

RF

IF

LMS

MUSIC

CMA

RLS

A/D

DSP

DAB

DOA

QAM

QPSK

AOA 
Signal to Interference and Noise Ratio

SINR

Single Input Multiple Output

SIMO

Minimum Mean Square Error

MMSE

Radio Frequency to Intermediate Frequency RF-to-IF

Intermediate Frequency to Base-band IF-to-BF

Frequency 


\section{Acknowledgement}

I would like to express my sincerest gratitude to my thesis supervisors, Professor Langis Roy and Professor Ian Marsland, for all of their insights and guidance. Without their help, I definitely will not be able to complete my research. In addition, I would like to thank N.C.I.T. for providing the funding and equipments for this research project. Furthermore, I would like to acknowledge Mr. Nagui Mikhail for his help and his advice on building the data capture system. Last but not least, I would like to thank my family and my girlfriend for their constant throughout my studies. 


\section{Introduction}

\subsection{Motivation}

As wireless networking devices have become more ubiquitous, the demand for higher bandwidth communication channels has increased dramatically. The idea of using antenna array, smart antennas, to mitigate interferences and to enhance channel capacity has been around for a long time [1]. However, because of limited computational power, such smart antenna enabled devices have only just started to be deployed in wireless communication systems. There are basically two types of smart antenna architectures: switched-beam antenna systems, and digital adaptive beam-former (DAB) systems. Currently, the switched-beam antenna system is the most widely deployed architecture because of its low computational costs. In a switched-beam system, each individual antenna beam is highly directional and the system selects the output, which results in the highest signal gain. In contrast, the more advanced DAB system is able to adjust the radiation pattern of the antenna array dynamically as the channel changes. However, because of the higher computational costs, DAB systems have only recently become economically viable for receivers.

The DAB system digitizes the baseband output of the individual antennas forming several streams of data samples; each of the data samples is linearly combined before it is fed into the receiver for further processing. There are two basic adaptive algorithm types used in DAB systems: blind adaptive algorithms, and non-blind adaptive algorithms. Blind adaptive algorithms do not rely on any training signals to converge. Two of the more popular algorithms are the Constant Modulus Algorithm (CMA) and the MUltiple 
SIgnal Classification (MUSIC) algorithm [2]. On the other hand, non-blind adaptive algorithms rely on some known symbols in the transmitted message to measure the characteristics of the channel. The Least Mean Square (LMS) algorithm and the Recursive Least Squares (RLS) algorithm are two of the most studied non-blind algorithms used in DAB systems [2|.

Along with the adaptive algorithm type, there are several beam-former architectures that may be considered. Typically, the beam-former is placed before the equalizer, and the error signal used to update the weights in the beam-former is generated based on the immediate output of the bean-former. As a result, the performance of the overall receiver is limited because at the output of the beam-former any temporal distortion cannot be equalized by the beam-former. One approach to rectify this problem is use the signal at the output of the equalizer to generate the error signal, which is used to update the taps in both the equalizer and the beam-former. Consequently, the adaptive algorithm can perform filtering in both temporal and spatial domains resulting in a lower bit error rate. There have been some researches done on the combined equalizer and beam-former architecture, which show the effects of varying different system parameters, such as the number of taps in the beam-former, the number of taps in the equalizer. and the step size of the equalizer, on the overall performance of the receiver.

As shown in this thesis, the combined equalizer and beam-former offers many benefits, which the traditional system fails to deliver. First of all, the overall acquisition range of the receiver can be improved. Secondly, the adaptive algorithm is able to combine multiple paths with large latency spreads to enhance the overall bit error rate performance of the receiver. Although the combined architecture offers a number of 
advantages over the traditional design, the architecture suffers from three inherent problems: sensitivity to the magnitude of the Mean Square Error (MSE) signal, slow convergence speed, and variable error feedback latency. Further studies are required to find solutions for these problems.

\subsection{Thesis Objectives}

There are three main objectives that this thesis will attempt to accomplish. Firstly, this thesis is going to examine how each of the systems parameters identified above is going to affect the convergence time of a combined beam-former and equalizer system. (Faster convergence for the algorithm means that the algorithm is better able to adapt to the changes in the channel environment.) Moreover, the selection of these parameters and trade-offs involving the computational complexities, and performances are also going to be discussed.

Secondly, this thesis will attempt to address some of the inherent problems of the combined beam-former and equalizer architecture, in particular the variable feedback error delay problem described above. It is going to discuss how to dynamically adjust the feedback latency of the error signal going to the beam-former to compensate for the variable error feedback latency introduced by the feedforward filter. There are two different architectures, which can be used to compensate for the variable error feedback latency. This thesis is going to demonstrate which architecture is superior in terms of both BER and convergence time.

Finally, this thesis will present a digital transmission system built to capture some real-life data to validate the result obtained in the simulation for the error signal feedback 
delay compensation system. The capture system consists of a single transmitter antenna, and two receiver antennas. The outputs of the two receiving antennas are downconverted and decomposed to their corresponding in-phase and quadrature-phase components, which are then sampled using an A/D converter board and stored in a computer for off-line processing. Using the capture system, several sets of real-life data in different channel environments are captured and the performance data of various beam-former architectures will be presented. Two channel environments are considered: a channel with little latency spread, and a channel with a large latency spread. These data sets will be used to verify some of the results obtained in the parameter selection simulations and to validate the performance gain obtained by using the error signal latency compensation system discussed.

\subsection{Thesis Outline}

This thesis is divided into seven chapters. Chapter one is the introduction of this thesis. Chapter two is divided into two major sections. The first section presents a general discussion of a digital transmission system with adaptive beam-forming and equalization. Detailed mathematical descriptions are given for the three major components of the digital transmission system: the transmitter, the channel, and the receiver. In the second section, two different beam-forming and equalization architectures are presented: the traditional beam-former architecture and combined beamformer and equalizer architecture. In Chapter three, a discussion on how to select an optimal set of parameters for the combined beam-former and equalizer follows an examination of the properties for both architectures. Finally, chapter three will look at some of the problems related to the combined beam-former and equalizer system. 
Chapter four investigates different solutions for the variable error feedback delay problem stated in the previous chapter. In its investigation of different solutions for the variable error feedback delay problem stated in the previous chapter, chapter four will discuss several error signal delay compensation systems and their simulations. Chapter five describes a digital transmission built to capture some real-life data in order to examine the performance gain of the combined beamformer and equalizer system demonstrated in chapters three and four. There are four parts to Chapter Five. The first part gives descriptions of the different components of the transmission system. The second and third parts present the adaptive algorithms selected for the receiver, as well as the format of the transmitted signal. The final part describes the test procedures, which will be used to generalize the data. Chapter six presents the environment in which the tests are conducted and shows the results obtained from the real-life data. Chapter seven summarizes the findings presented in the previous chapters and a list of future works. 


\section{A Smart Antenna Enabled Digital Transmission System Model}

A single input multiple output (SIMO) digital transmission system improves bit error rate by combining signals from multiple antennas. Figure 1 shows the four major components in a SIMO digital transmission system model: the transmitter, the channel, the demodulator/sampler, and the smart antenna signal processor.

The transmitter model takes a stream of message bits and converts the bits into data symbols. The data symbols are filtered, using the pulse shape filter, and modulated before they are passed into the transmission channel. The demodulator/sampler receives the transmitted symbol and removes the carrier; afterwards, the baseband signal is sampled to generate a stream of complex digital data and the stream is fed into the smart antenna signal processor. The first stage of the smart antenna signal processor is the beam-former, which performs a weighted sum operation on the sampled data to generate

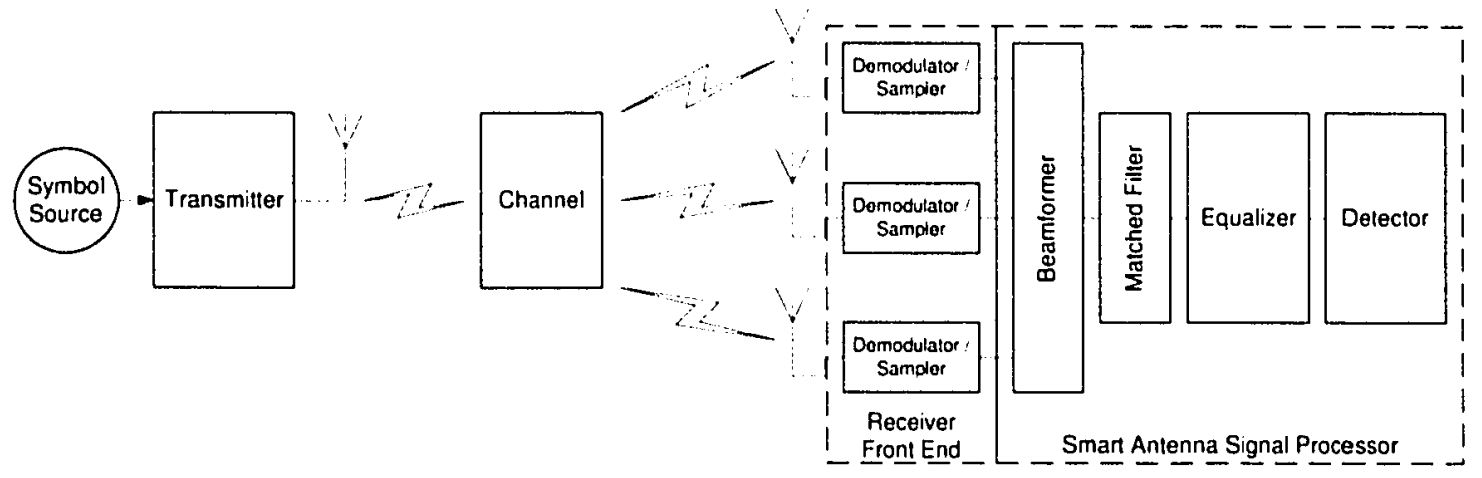

Figure 1. A smart antenna enabled digital transmission system model consists of four major components: the transmitter, the channel. the receiver / sampler, and the smart antenna signal processor. 
a single data stream. The weights in the beam-former are updated adaptively to track the changes in the channel. Once the signal is beam-formed, the equalizer will attempt to remove the temporal distortion of the signal.

In the following section, the transmitter, the channel, the demodulator/sampler and the smant antenna signal processor will be described in detail. A mathematical model, which illustrates how the transmitted message travels from the transmitter to the smart antenna signal processor, will also be developed. In addition, some of the problems with the traditional beam-former will be analyzed and an alternate beam-former design will be explored.

\subsection{Transmitter Model}

A simple transmitter can be modeled using three separate sub-blocks: a symbol mapper, a pulse shape filter, and a modulator. Figure 2 shows the structure of a transmitter; the transmitter takes the message bits generated by the symbol sources and modulates the symbol before it is transmitted in the channel.

The symbol source generates a message of $N$ symbols, long denoted by

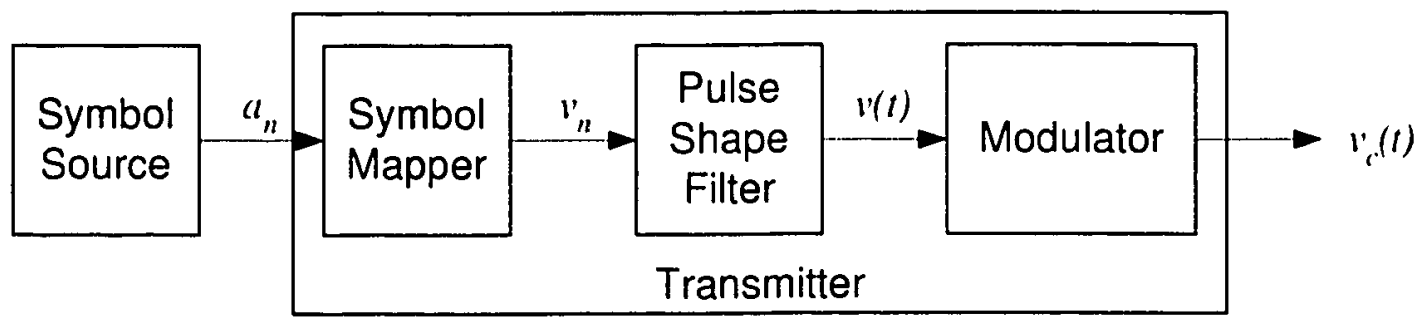

Figure 2. A simple transmitter consists of four basic building blocks: a symbol source, a symbol mapper, a pulse shape filter, and a modulator. 
$\bar{a}=\left[a_{0}, a_{1}, \ldots, a_{N-1}\right]$, and each symbol consists of $m$ bits. In the case of QPSK, each symbol is made of two bits, where $a_{n} \in\{00,01,10,11\}$ for $n \in\{0,1, \ldots, N-1\}$. The first $N_{1}$ symbols are training symbols embedded in the message. The resulting baseband signal, $V(t)$, is denoted by the pulse train,

$$
v(t)=\sum_{n=0}^{N-1} v_{n} h_{T}(t-n T)
$$

where $h_{r}(t)$ is the pulse shape filter, $T$ is the symbol duration, and $v_{n}$ is the signal constellation that corresponds to $a_{n}$ given by

$$
v_{n}=\operatorname{SM}\left(a_{n}\right)
$$

where the function, $\operatorname{SM}(m)$, is the symbol mapper function. For QPSK, $\operatorname{SM}(m)$ can be expressed using the lookup table below:

$$
\mathrm{SM}(m)=\left\{\begin{array}{cc}
1 & m=00 \\
j & m=01 \\
-j & m=10 \\
-1 & m=11
\end{array}\right.
$$

The mapped symbol $v_{n}$ is multiplied by the pulse shape filter $h_{r}(t)$. A popular choice of $h_{r}(t)$ is the root raised-cosine filter and the impulse response of the filter is given by

$$
h(t)=\frac{4 \alpha}{\pi \sqrt{T}} \frac{\cos \left(\frac{(1+\alpha) \pi t}{T}\right)+\frac{T}{4 \alpha t} \sin \left(\frac{(1-\alpha) \pi t}{T}\right)}{1-\left(\frac{4 \alpha t}{T}\right)^{2}}
$$




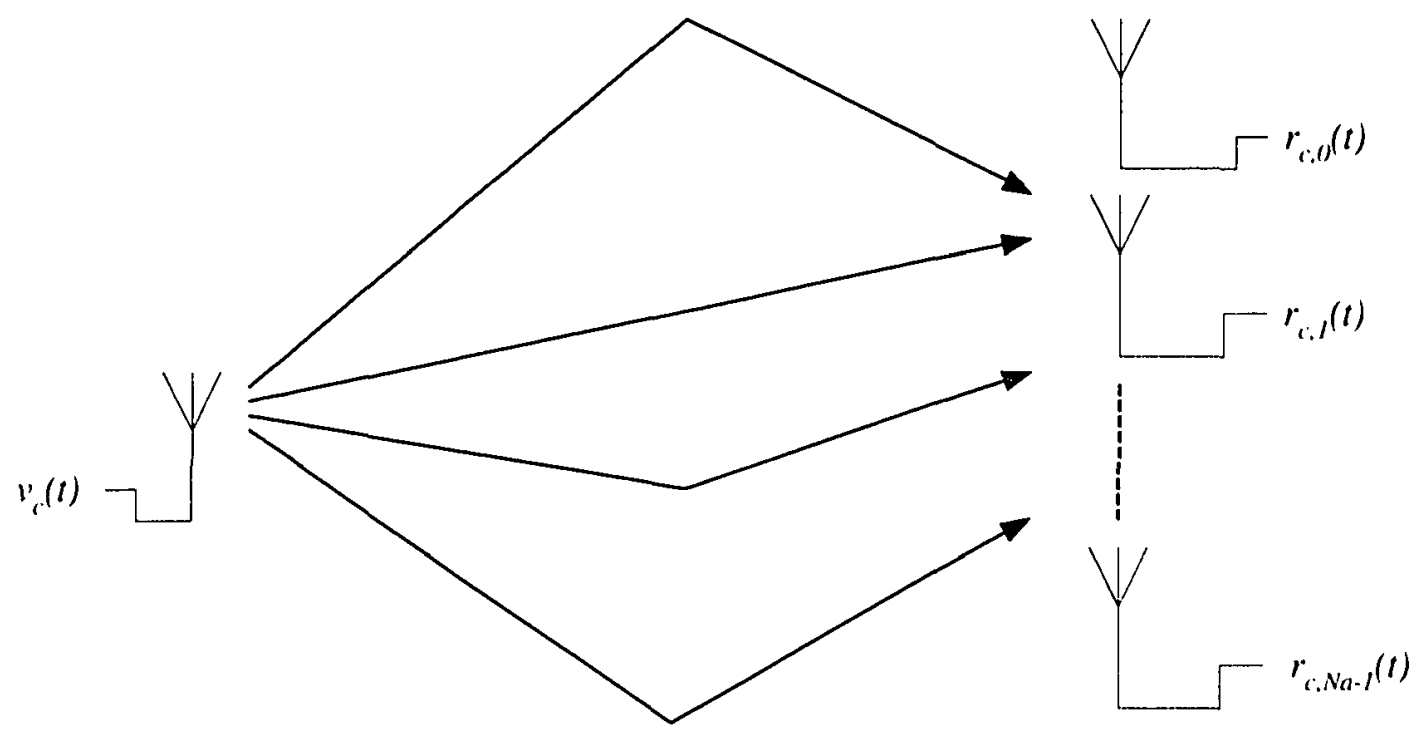

Figure 3. The transmitted signal travels $\mathrm{K}$ different paths to reach the receiver antennas; the attenuation of each path follows the Rayleigh distribution.

where $\alpha \in[0,1]$ is the roll off factor, which controls the bandwidth of the transmitted message. [3]

The baseband signal is then modulated with the carrier signal, and the transmitted bandpass signal is given by

$$
v_{c}(t)=\operatorname{Re}\left\{v(t) \sqrt{2} e^{j 2 \pi f_{1} t}\right\}
$$

where $f_{i}$ is the frequency of the carrier.

\subsection{Channel Model}

Figure 3 shows the multi-path channel environment: the transmitted signal propagates to the $N_{\mathrm{A}}$ antennas via $K$ different signal paths. The signal received at antenna $a$ is given by 


$$
r_{c, a}(t)=\sum_{k=0}^{K-1} \alpha_{k} v_{c}\left(t-\tau_{k, a}\right)+w_{c \cdot a}(t)
$$

where $K$ is the number of signal paths in the channel, $\alpha_{k}$ is the attenuation along signal path $k$, which has a Rayleigh distribution of $\mathrm{E}\left[\alpha_{k}\right]=1, \tau_{k, a}$ is the propagation delay of the signal path $k$ going to antenna $a$, and $w_{c \cdot a}(t)$ is the additive Gaussian noise introduced by the channel and the antenna.

The propagation delay of the signal can be modeled by separating $\tau_{k . a}$ into two components,

$$
\tau_{k, a}=\tau_{k}+\tau_{k . a}^{\prime}
$$

where $\tau_{k}$ is the intrinsic delay of the $k$-th signal path (the propagation time from the transmitter to the receiver antenna array along the $k$-th signal path), and $\tau_{k, a}^{\prime}$ is the additional time for the signal wave front to travel to $a$-th antenna. The intrinsic delay of the signal varies from path to path: however, it is bound by $\tau^{\prime}$. and $\tau^{\prime \prime}$, where $\tau^{\prime}$ is the minimum path delay, and $\tau^{\prime \prime}$ is the maximum path delay. The delay spread of the channel is denoted by the absolute difference between the two variables, $\left|\tau^{\prime \prime}-\tau^{\prime}\right|$. Figure 4 shows the derivation of the $\tau_{k, a}^{\prime}$ using a simple linear array model $[4]$. One can determine the flight time of the signal, $\tau_{k . a}^{\prime}$, by finding the extra distance required to travel to antenna element $a$ divided by the speed of the wave front. Thus, $\tau_{k, a}^{\prime}$ is given by 


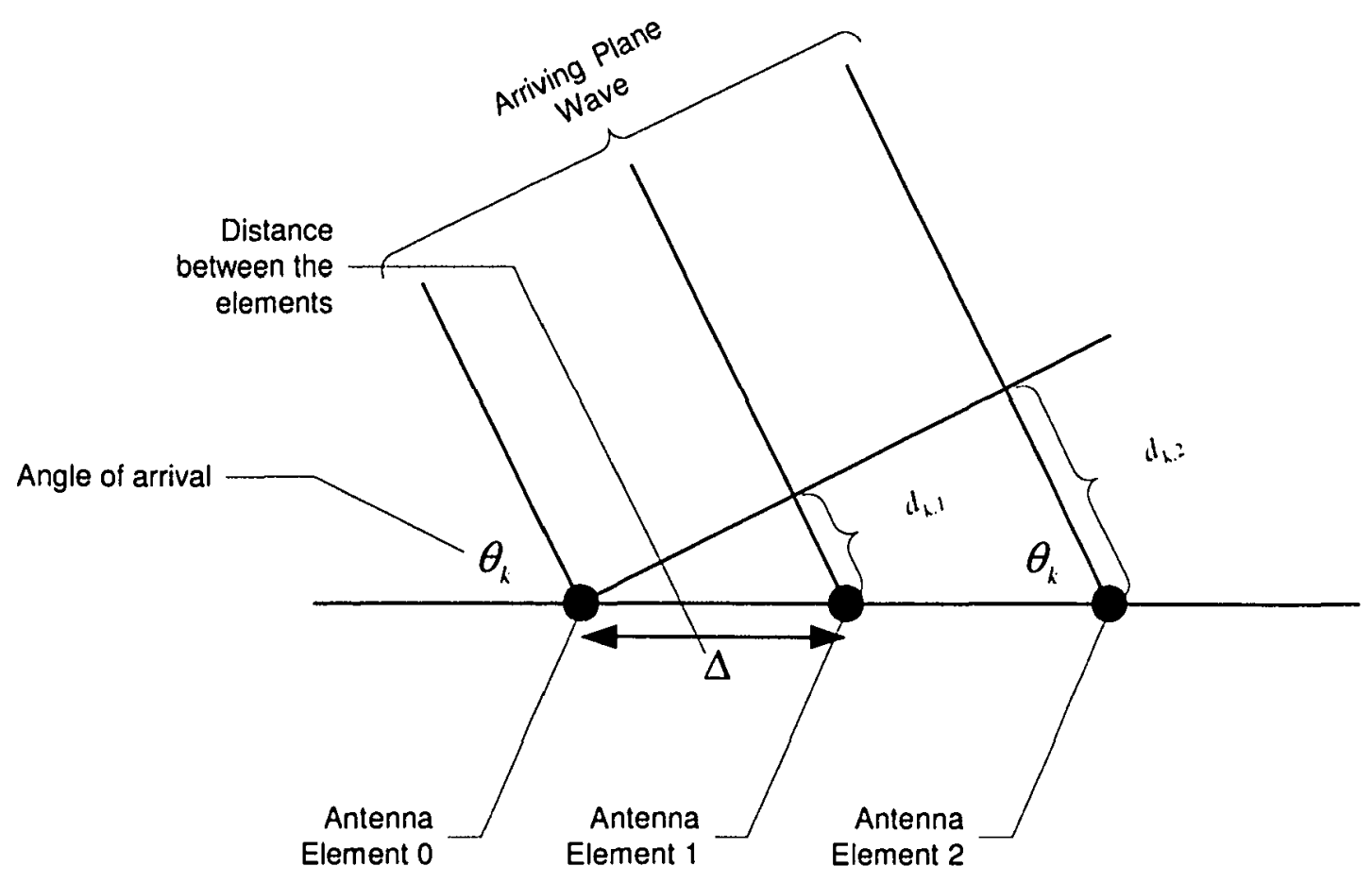

Figure 4. The diagram shows how simple trigonometry can be used to approximate the value of $d_{k, a}$.

$$
\tau_{k, a}^{\prime}=\frac{d_{k . a}}{c}
$$

where $d_{k, a}$ is the extra distance traveled by the wave front, and $c$ is the speed of light.

Using simple geometry, the distance, $d_{l, a}$, is shown to be,

$$
d_{k, a}=a \Delta \cos \theta_{k}
$$

where $a$ is the antenna number from left to right (assuming that the left on most antennas is antenna 0 ), $\Delta$ is the distance between each antenna element (assuming that the antennas are uniformly distributed along the same axis), and $\theta_{k}$ is the angle of arrival of the signal path $k$. Thus, $\tau_{k, a}$ can be expressed as: 


$$
\tau_{k, a}=\tau_{k}+\frac{d_{k, a}}{c}=\tau_{k}+\frac{a \Delta}{c} \cos \theta_{k}
$$

Typically, $\Delta$ is expressed as a fraction of the carrier wavelength,

$$
\Delta=\rho \lambda,
$$

where $\rho$ is a fraction of the wavelength $\lambda$, which is given by

$$
\lambda=\frac{c}{f_{c}}
$$

Finally, the total propagation delay is expressed by the following equation,

$$
\tau_{k, a}=\tau_{k}+a \frac{\rho}{f_{c}} \cos \theta_{k}
$$

\subsection{Receiver Model}

Figure 5 shows the structure of a receiver front end, which is modeled using three separate components: a demodulator, a low pass filter, and a sampler. The demodulator multiplies the received signal from the antenna with a locally generated carrier to obtain a demodulated signal. The output of the demodulator is

$$
r_{o, a}(t)=r_{c, a}(t) \sqrt{2} e^{-j 2 \pi f_{i} t} e^{j \phi_{t}}
$$

where $r_{c, a}(t)$ is the signal from the $a$-th antenna, and $\phi_{c}$ is the phase difference between the transmitter carrier and the receiver carrier. At this stage, no attempt is made to correct the phase difference, which will be handled eventually by the beam-former down- 


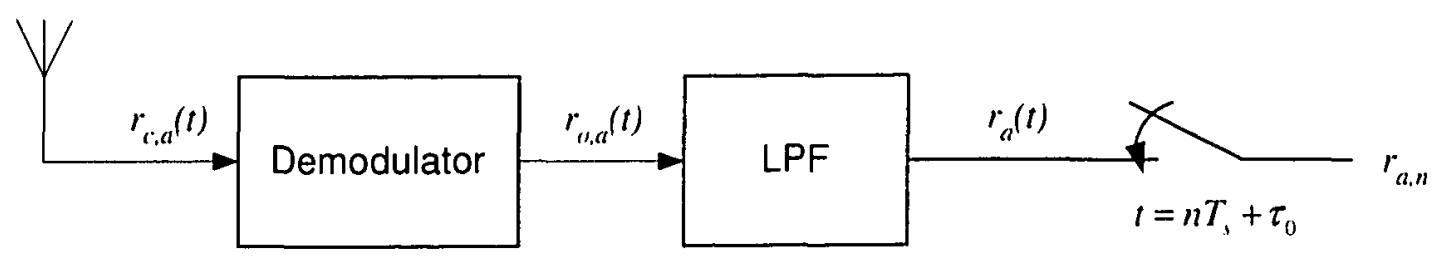

Figure 5. The receiver front end demodulates the bandpass signal by multiplying it with a locally generated version of the carrier, and the resulting baseband signal is low-pass filtered to remove any high frequency noise, before it is sampled by the sampler.

stream. By substituting $r_{r, a}(t)$ with the definition presented in (6), the follow equation is obtained

$$
r_{o, a}(t)=\left[\sum_{k=0}^{K-1} \alpha_{k} v_{c}\left(t-\tau_{k, a}\right)+w_{c, a}(t)\right] \sqrt{2} e^{-j 2 \pi f_{c} t} e^{j \phi_{c} .} .
$$

With further substitution and algebraic manipulation, the above equation can be expressed as

$$
\begin{aligned}
& r_{o, a}(t)=\sum_{k=0}^{K^{\prime}-1} \alpha_{k} \operatorname{Re}\left\{v\left(t-\tau_{k, a}\right) \sqrt{2} e^{j 2 \pi f_{i}\left(t-\tau_{i, a}\right)}\right\} \sqrt{2} e^{-j 2 \pi f_{,} t} e^{j 0_{i}}+ \\
& w_{c \cdot a l}(t) \sqrt{2} e^{-j 2 \pi f_{1} t} e^{j \varphi_{c}} \\
& =\sum_{k=0}^{K-1} \alpha_{k} \nu\left(t-\tau_{k, a}\right) e^{-j 2 \pi f_{i} \tau_{k, n}} e^{j \phi_{i}}+ \\
& \sum_{k=0}^{K-1} \alpha_{k} v\left(t-\tau_{k, a}\right) e^{-j+\pi f_{,} t} e^{j 2 \pi f_{,} \tau_{k, a}} e^{j o_{1}}+w_{o, a}(t)
\end{aligned}
$$

where $w_{c, a}(t)=w_{c, a}(t) \sqrt{2} e^{-j 2 \pi f . t} e^{j \phi_{i}}$ is the demodulated noise and the second term in (16) is the high frequency component of the demodulated signal, which is subsequently removed by the low pass filter. 
The low pass filter serves two major purposes in the receiver system: to prevent aliasing during the sampling process, and to eliminate out-of-band noise. The low pass filter $W$ must be greater than or equal to the bandwidth of the lowpass transmitted signal, which for the root raised cosine filter is $\frac{1+\alpha}{2 T}$, where $T$ is the symbol duration and $\alpha$ is the roll-off factor of the filter. The output of the low pass filter can be expressed by

$$
r_{a}(t)=r_{o, a}(t) * h_{l, p}(t)
$$

where $h_{t, \mu}(t)$ is the impulse response of the lower pass filter and * denotes convolution. After the appropriate substitutions, the above equation can be re-written as

$$
r_{a}(t)=\sum_{k=0}^{K-1} \alpha_{k} v\left(t-\tau_{k, a}\right) e^{-j 2 \pi f_{1} \tau_{k a} a} e^{j \phi_{c}}+w_{a}(t)
$$

where $w_{a}(t)=w_{t, a}(t) * h_{l,}(t)$ is the filtered noise.

After the signal is demodulated and filtered, it is sampled at rate $1 / T_{1}$, where $T_{1}=T / \eta$ and $\eta$ is the sampling rate of the system. before it is processed digitally by the beam-former. (In the simulated system, the sampling rate is 8 samples per symbol.) Assuming that sampling begins after the clock is recovered, the samples obtained are further delayed by $\tau_{0}$ which is equal to $\tau^{\prime}$ for the system. The $n$-th sample is given by

$$
r_{a, n}=r_{a}\left(n T_{s}+\tau_{0}\right)
$$

By substituting (1), (5) and (18) into (19), one obtains 


$$
\begin{aligned}
r_{a, n}= & \sum_{k=0}^{K-1} \alpha_{k} v\left(n T_{s}+\tau_{0}-\tau_{k, a}\right) e^{-j 2 \pi f_{c} \tau_{k, a}} e^{j \phi_{c}}+w_{o, a}\left(n T_{s}+\tau_{0}\right) \\
= & \sum_{k=0}^{K-1} \alpha_{k} \sum_{m=0}^{N-1} v_{m} h_{T}\left(n T_{s}+\tau_{0}-\tau_{k, a}-m T\right) e^{-j 2 \pi f_{c} \tau_{k, a}} e^{j \phi_{i}}+w_{a, n} \\
= & \sum_{m=0}^{N-1} v_{m} \sum_{k=0}^{K-1} \alpha_{k} h_{T}\left((n-\eta m) T_{s}+\left(\tau_{0}-\tau_{k, a}\right)\right) e^{-j 2 \pi f_{r}\left(\tau_{k}+a \frac{\rho}{f_{i}} \cos \theta_{k}\right)} e^{j \phi_{i}}+ \\
& w_{a, n}
\end{aligned}
$$

By defining

$$
h_{k}=\alpha_{k} e^{j \phi_{i}} e^{-j 2 \pi f_{c} \tau_{k}}
$$

and

$$
\phi_{k . a}=-2 \pi a \rho \cos \theta_{k}
$$

(20) can be further simplified to

$$
r_{a, n}=\sum_{m=0}^{N-1} v_{m} \sum_{k=0}^{K-1} h_{k} h_{r}\left((n-\eta m) T_{s}+\left(\tau_{0}-\tau_{k, a}\right)\right) e^{j \varphi_{\alpha . a}}+w_{a, n}
$$

where $h_{k}$ has a complex Gaussian distribution, with a zero mean and variance of $\left.\mathrm{E}|| h_{k}\right|^{2} \mid=1$, and $\theta_{k}$.is uniformly distributed over $[0,2 \pi]$.

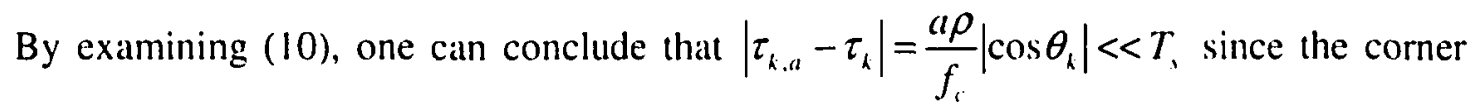
frequencies are typically much larger than the sampling rate, so $\tau_{h, a} \cong \tau_{k}$. Therefore, (23) can be re-written as 


$$
r_{a, n}=\sum_{m=0}^{N-1} v_{m} \sum_{k=0}^{K-1} h_{k} h_{T}\left((n-\eta m) T_{s}+\left(\tau_{0}-\tau_{k}\right)\right) e^{j \phi_{k, a}}+w_{a, n} .
$$

In summary, (24) shows the relationship between the transmitted symbols, $\left\{v_{m}\right\}$, and the received samples, $\left\{r_{a, n}\right\}$ : hence, (24) can be used as the discrete-time channel model for the system. The noise component of the system is independent and has zero mean: the power spectrum density is defined as follows,

$$
\left.\mathrm{E}|| w_{a, n}\right|^{2} \mid=2 W N_{0}
$$

\subsection{Smart Antenna Signal Processor}

After the receiver demodulates the signal, the complex sampled baseband signal is fed into the smart antenna signal processor for beam-forming and equalization. Figure 6 shows the basic structure of a smart antenna signal processor, which consists of a beamformer, a matched filter, and an equalizer. The beam-former studied in this thesis is a delay and sum network: the antenna samples are linearly combined to form a single sampled stream, which passes through the matched filter. After the signal is filtered, the equalizer suppresses any temporal distortion remaining in the signal.

In this section, the mathematical model for each of the components-the beamformer, the matched filter, and the equalizer-is described. In addition, the adaptive algorithms used to update the taps in the beam-former and the equalizer are presented. 


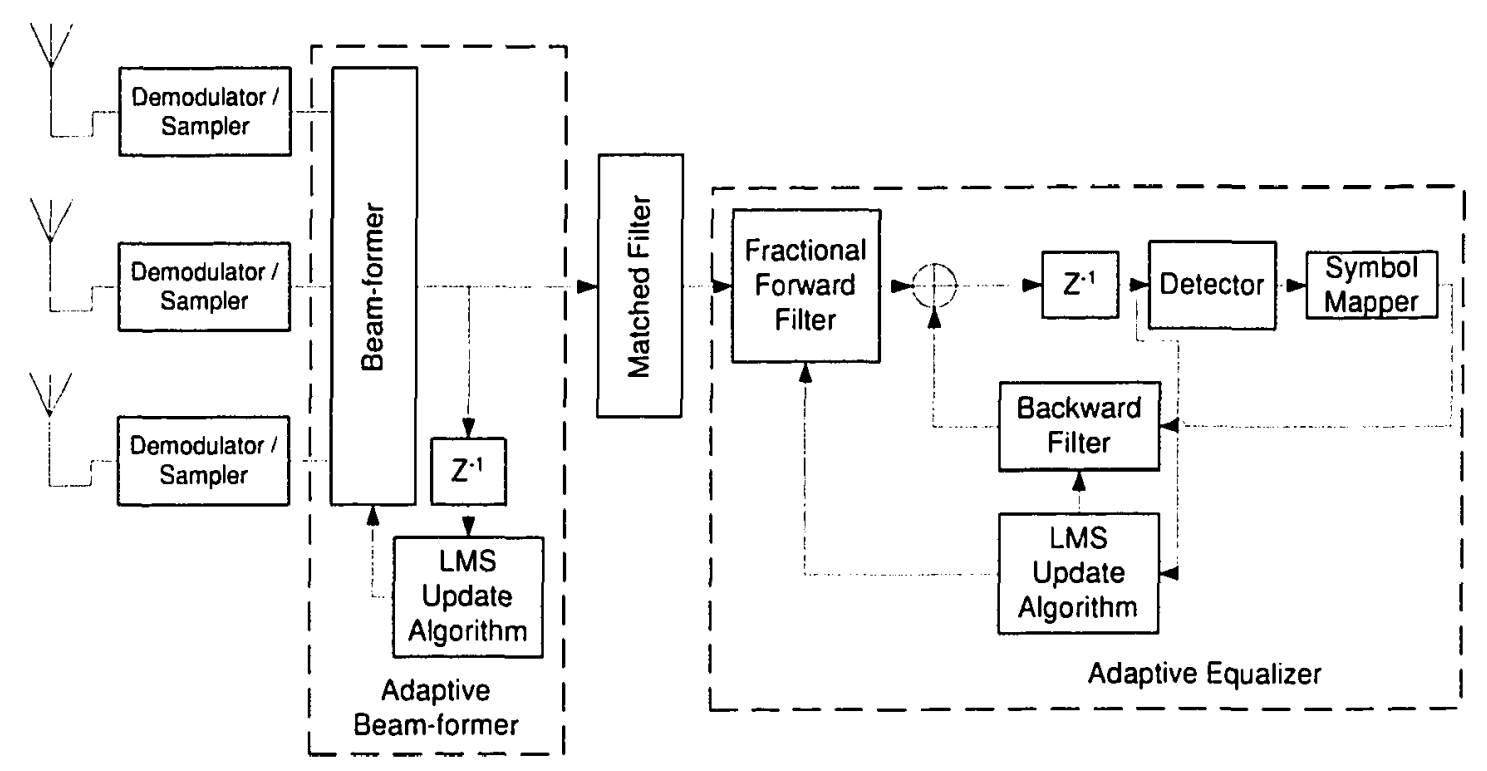

Figure 6. A traditional beam-former uses the immediate output of the beam-former to generate the necessary error signal.

\subsubsection{Beam-former Model}

The digital beam-former is a weighted delay and sum network, which can be modeled using a bank of finite impulse response (FIR) filters. The complex received samples are fed into the FIR filters, and the filter outputs are summed together. Figure 7 shows the structure of an adaptive digital beam-former.

Mathematically, the digital beam-former can be described using

$$
x_{a, n}=\sum_{l=0}^{l_{H F}-1} \rho_{a, l} r_{a, n-l}
$$

and

$$
x_{n}=\sum_{a=0}^{N_{A}-1} x_{a, n}
$$




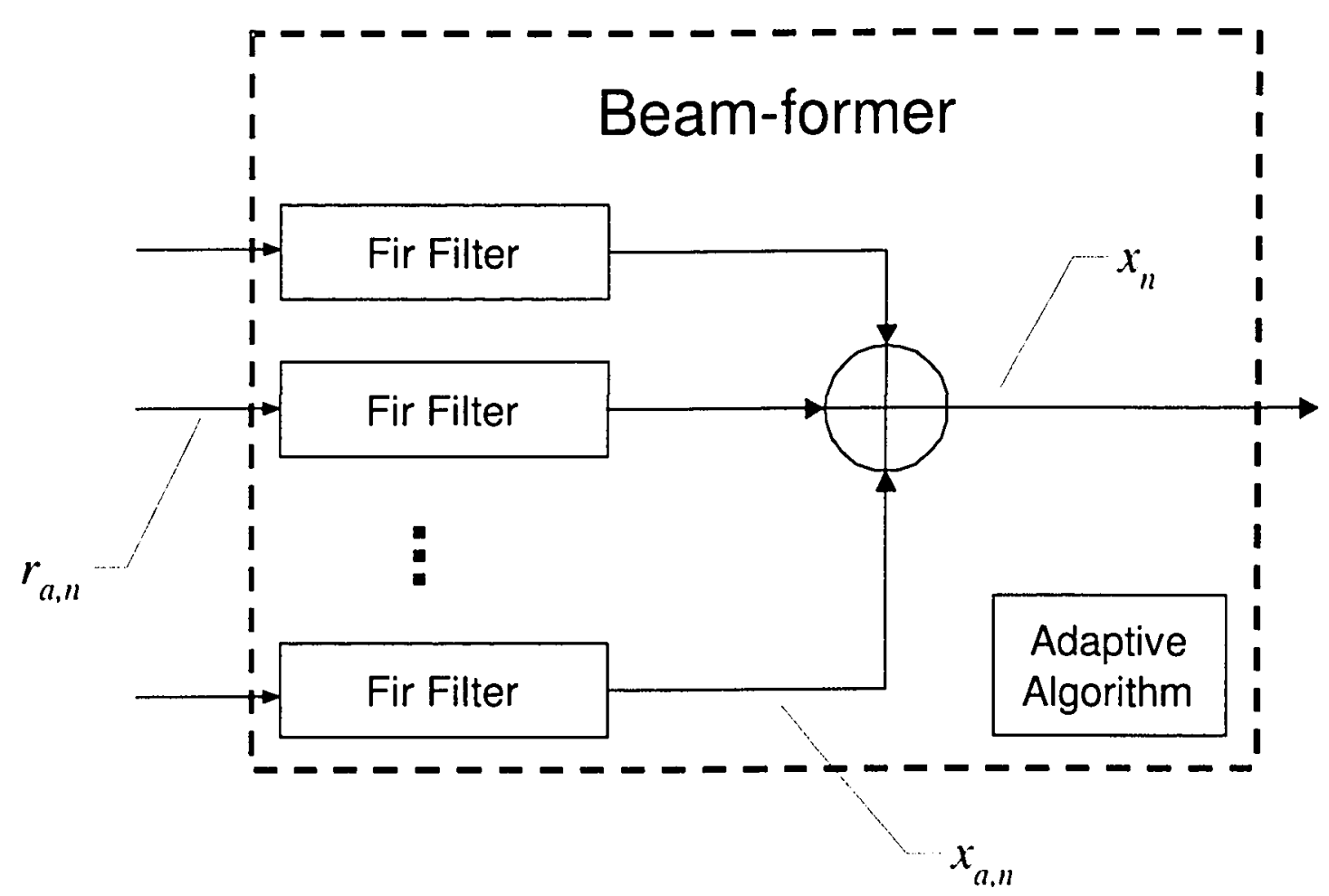

Figure 7. An adaptive digital beam-former consists of a weighted sum network to generate a single stream of sampled data.

where $r_{a, n}$ is the $n$-th complex sampled baseband output from the $a$-th antenna and $\rho_{a, l}$ is the $l$-th tap of the $a$-th FIR filter $|6|$. The total number of taps, $L_{k r}$, is determined by the sampling rate and time spread of the received signal. A detailed discussion of how $L_{H F}$ should be selected is presented in Chapter 3.2. The outputs of the $N_{A}$ FIR filters, $x_{n, a}$, are added together to form $x_{n}$, which is the output of the beamformer.

To track the changes in the channel, the tap weights, $\rho_{a, l}$, are updated regularly. There are many different adaptive algorithms for updating the taps, such as CMA. 
MUSIC, RLS, and LMS [2][7][8]. This thesis will focus on the minimum mean square error (MMSE) based algorithms, particularly, the LMS algorithm, because it is the simplest of all the algorithms mentioned above and it has reasonable convergence characteristics. Using the steepest decent method, the update equation for the $\rho_{a, l}(m+1)$ using LMS is expressed by (The derivation of the equation is not given in this paper but it is presented in [9] and [10])

$$
\rho_{a, l}(m+1)=\rho_{a, l}(m)-2 \mu r_{a . \xi m-l}\left(x_{\xi m}-v_{\xi n}\right)^{*}
$$

where $\rho_{a, l}(m)$ is the value of the filter tap of the $m$-th iterations, $\mu$ is the update step size, $r_{a, 5_{m-1}}$ is the $\xi_{n-l}-1$ th incoming data sample, assuming that the algorithm updates the tap value every $\xi$-th samples, and $v_{5, n}$ is the known sampled value, usually the training sequence. In the above equation, the error signal is generated using the direct output of the beam-former. Section 2.5.2 will describe an alternate error equation, which is shown to improve the SNR characteristic of the receiver system.

\subsubsection{Matched Filter Model}

In order to obtain maximum ISI rejection, a matched filter is applied to the beamformed signal. The matched filter is deliberately placed after the beam-former because the beam-former has corrected the phase of the received samples; hence, additional phase compensation is not required before the signal is down-sampled. When the pulse-shape filter auto-convolutes, $h_{a}(t)=h_{\sqrt{a}}(t)^{*} h_{\sqrt{a}}(t)$, the maximum amplitude, or the optimal sampling point, occurs when $h_{a}(t=0)|3|$. However, the received signal is delayed by an arbitrary amount, depending on the latency of the channel; as a result the optimal 
sampling point will also be shifted. Typically, a symbol time recovery circuit is required to find the optimal sampling point. In the case of a beam-formed system, non-blind adaptive algorithms will ensure that the received signal has the same phase and delay as the known desired signal; consequently, the optimal sampling point is the same under different channel environments. The operation of the matched filter can be modeled using the following equation,

$$
y_{n}=\sum_{l=-L_{3, t}}^{l_{3 / t}} h_{\sqrt{n}, l} x_{n-l}
$$

where $h_{\sqrt{a} . l}=h_{\sqrt{a}}\left(I T_{s}\right)$ and $h_{\sqrt{a}}(n)$ is the same as the root raised-cosine filter shown in (4). In addition to filtering, the matched filter model also down-samples the incoming signal before it is fed into the equalizer. The down sampling is defined by

$$
y_{c^{\prime}}=y_{\beta n}
$$

where $\beta$ is the down sampling factor. (In most of the simulations performed using the transmission system, the $\beta$ is set to 4.$)$

\subsubsection{Equalizer Model}

The equalizer used in the digital transmission system model studied in this thesis is a simple decision feedback equalizer |3|: the feedforward filter is fractionally spaced, while the feedback filter is symbol spaced. The structure of the equalizer is shown in Figure 8 . 


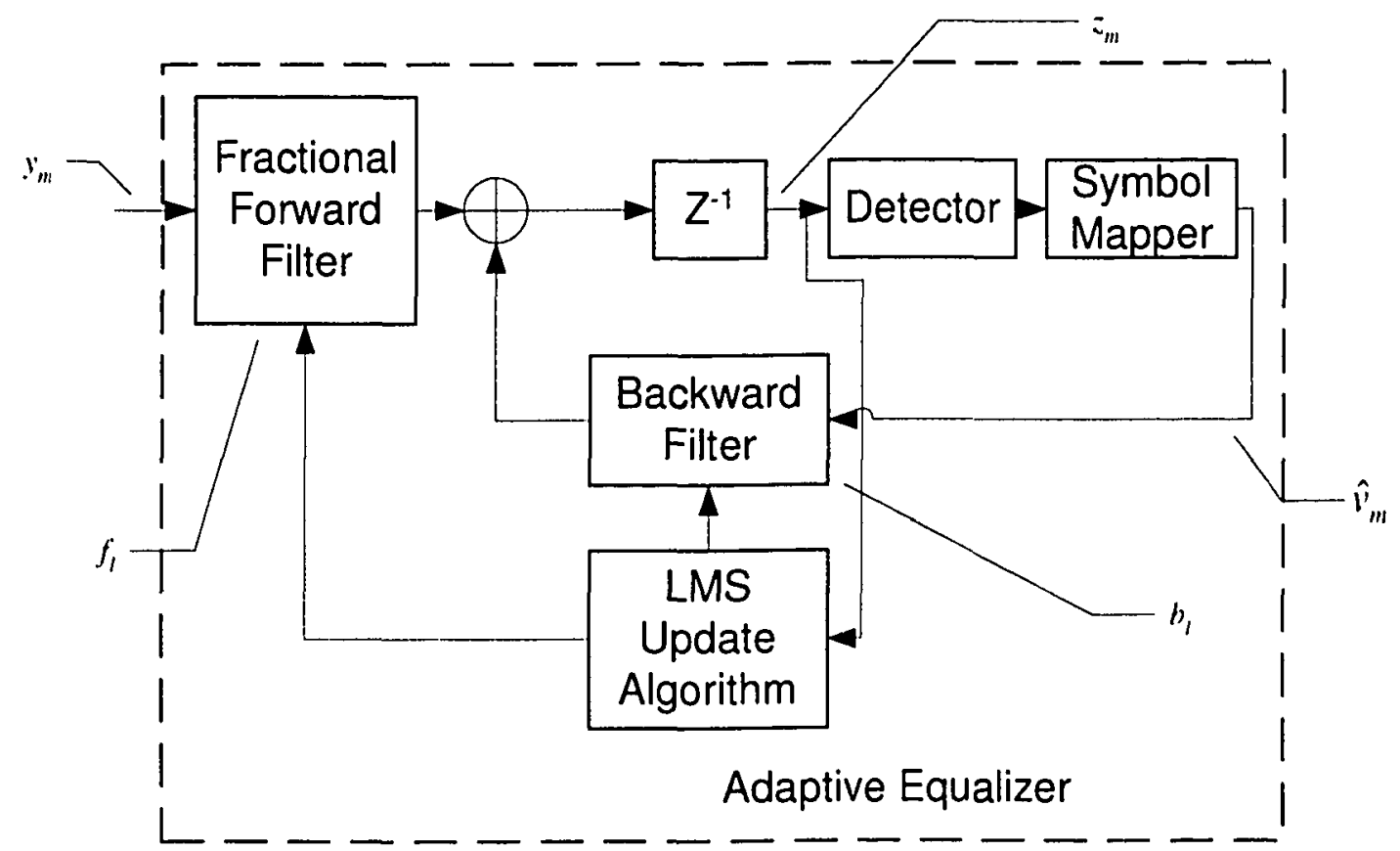

Figure 8. The structure of an adaptive equalizer consists of two equalizers: the feedforward filter, and the feedback filter. Both equalizers are adaptive and their tap weights are updated using the same error signal.

The behavior of the equalizer can be modeled using

$$
\bar{z}_{m}=\sum_{l=0}^{l_{+\mu}-1} f_{l}^{(m)} y_{x^{m-l}}-\sum_{l=0}^{l_{m+}-1} b_{l}^{(m)} \hat{\imath}_{m-l}
$$

where $y_{x m-l}$ is the output of the matched filter, $\chi$ is the down sampling factor of the feedforward filter, $L_{r r}$ and $L_{r H}$ are the lengths of the feedforward and the feedback filter, $f_{l}^{(m)}$ and $b_{l}^{(m)}$ are the tap weights for the feedforward and the feedback filter, and $\hat{v}_{m-l}$ is the estimation of the transmitted symbols. $\hat{v}_{m-1}$ is the rectified version of $z_{m}$, and is given by

$$
\hat{v}_{m-l}=\operatorname{SM}\left(\hat{z}_{m}\right)
$$


where $\hat{z}_{m}$ is given by

$$
\hat{z}_{m}=\arg \max _{a} \mid z_{m}-S M[a]^{2}
$$

For the equalizer to adapt to the channel changes, the tap weights in both the feedforward and feedback filter branches are updated using an adaptive algorithm. (The same set of adaptive algorithms described above for the beam-former can be used in the equalizer as

well.) If the LMS algorithm is used, the update equation for the tap weights, $f_{l}^{(m)}$ and $b_{l}^{(m)}$, can be described in the following set of equations:

$$
\begin{aligned}
& f_{l}^{(m+1)}=f_{l}^{(m)}-2 \mu y_{x^{m-1}}\left(z_{m}-v_{m}\right)^{*} \\
& b_{l}^{(m+1)}=b_{l}^{(m)}-2 \mu \hat{v}_{m-1}\left(z_{m}-v_{m}\right)^{*}
\end{aligned}
$$

\subsection{Combined beam-former and equalizer}

The beam-former presented in Chapter 2.4.1 is a more traditional beam-former design because the tap weights in the beam-former are updated based on the immediate output of the beam-former. There are several problems with this structure: especially, the fact that the adaptive algorithm used is a non-blind MMSE based algorithm. The following section will examine some of the weaknesses of the traditional beam-former: afterwards, an alternative beam-former design, based on the LMS adaptive algorithm. which attempts to resolve some of the problems with the traditional beam-former, based a non-blind MMSE based algorithm, will be discussed. 


\subsubsection{Problems with the Traditional Beam-former}

The traditional beam-former design has a major limitation because for the MMSE based algorithm to produce a stable non-zero solution, the incoming signals and the "desired" signal must be synchronized. In the case of the traditional beam-former using LMS, one of the $r_{a, n}$ must be correlated with the "desired" signal, $v_{5 m+1}$; otherwise, the beam-former will not acquire the signal properly. This limitation creates two problems. First of all, in order to reduce the computational requirement of the beam-former, the length of the individual FIR filters, $L_{B r}$, tends to be quite short; hence, given that the latency of the channel, $\tau_{k, l}$, is unknown, it is possible to have none of the $r_{a, n-l}$, where $l$ is an integer from 0 to $L_{H r}$, to be correlated with $v_{5 m+1}$. Consequently, the LMS algorithm will fail to converge. Secondly, in a multi-path channel environment, $\tau_{k, a}$ for different paths can vary significantly. Because the LMS algorithm cimnot lock onto signals that are not correlated with the "desired" signal, any signal arriving at the receiver via a longer signal path will be treated as "noise", from the algorithm point of view. Typically most of the energy of the transmitted signal arrives at the receiver via the shortest signal path. For cases in which the shortest path is blocked, the LMS algorithm will not be able to combine signals arriving via the longer paths, which has a $\tau_{\text {h.a }}$ greater than $T_{s} \cdot L_{k F}$ : therefore, the effecliveness of the beam-former is reduced. One approach that can address both of these problems is to make the length of the FIR filters, $L_{n r}$, longer. In order for this solution to work, the minimum length of $L_{B r}$ is defined by

$$
L_{B F} \geq\left\lceil\frac{\max \left(\tau_{k, a}\right)}{T_{s}}\right\rceil
$$


where $T_{s}$ is the sampling rate of the system. As a result, the FIR filter can be quite long as the $\max \left(\tau_{k, a}\right)$ becomes very large or as symbol rates increase and $T_{s}$ becomes very small; consequently, the computational requirement of the beam-former increases significantly.

\subsubsection{Combining the Beam-former and Equalizer}

Given that the beam-former and the equalizer can use the same adaptive algorithm to update tap weights, another approach for resolving the problem stated in the previous section is to use the error signals generated by the equalizer to update the beam-former as well. Because the matched filter down-samples the beam-formed signal before it is fed into the equalizer, the minimum length of the feedforward filter, $L_{r+}$, becomes

$$
L_{F F} \geq\left\lceil\frac{\max \left(\tau_{k, a}\right)}{\beta \cdot T_{s}}\right\rceil
$$

where $\beta$ is the down-sampling factor of the matched filter. Since $L_{\mu, F}$ is $\beta$ times smaller than $L_{B F}$ and there is only one feedforward filter, as opposed to $N_{A}$ FIR filters in the beam-former, the computational cost for making

$$
L_{F F}=\left\lceil\frac{\max \left(\tau_{k, a}\right)}{\beta \cdot T_{s}}\right\rceil
$$

is much less ( $\beta N_{A}$ times smaller than achieving the minimum bound of $L_{B r}$ ). As a result, using the error signal to update the tap weights in the beam-former is a good trade off between additional computational costs and beam-former effectiveness. 


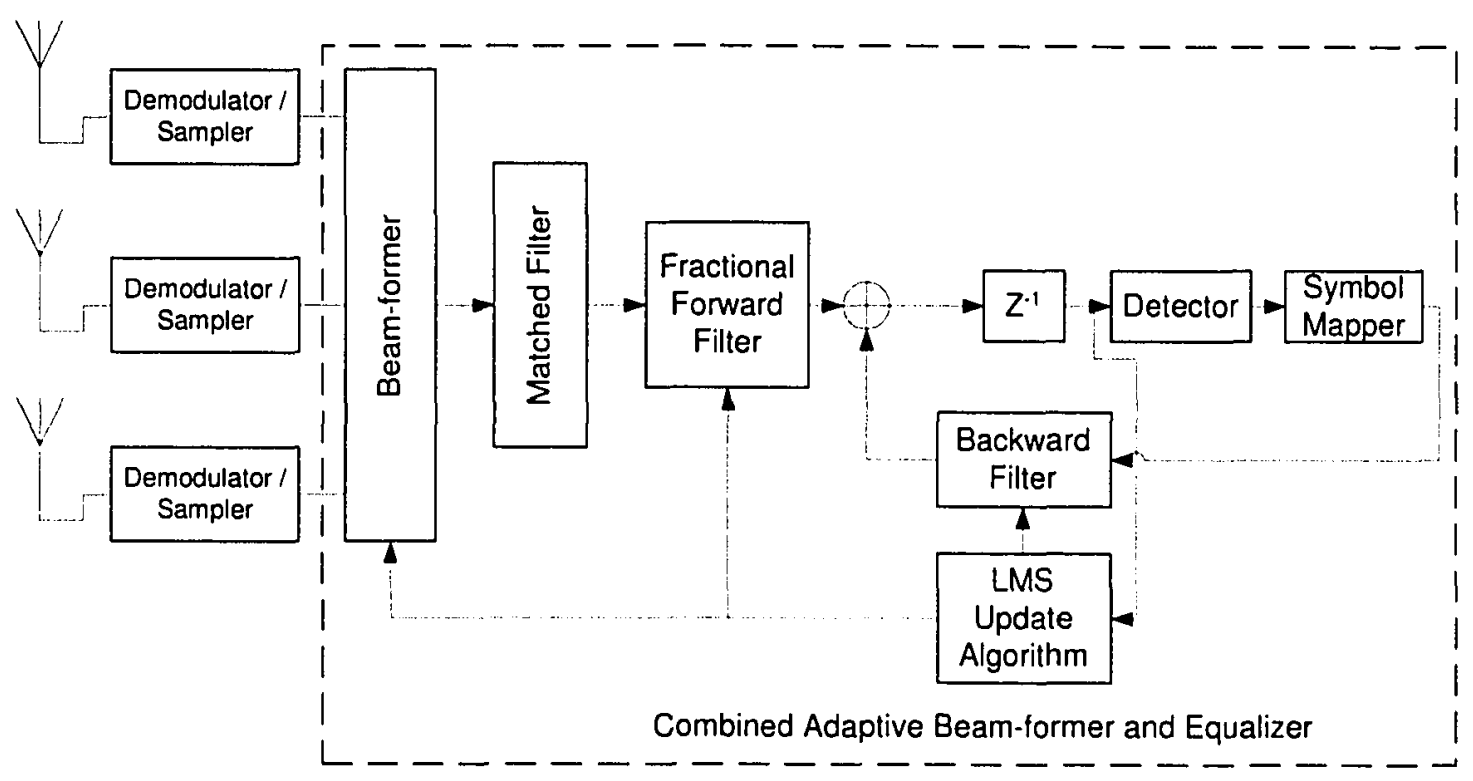

Figure 9. For the combined beam-former and equalizer architecture, the error signal for both the beam-former and equalizer is produced by comparing the output of the equalizer against the desired signal.

Figure 9 shows the basic structure of a combined beam-former and equalizer: the error signal generated for the equalizer is fed to the beam-former to update the tap weights in the beam-former. Because the same error signal is used for both the beamformer and the equalizer, the equation (28) can be re-written as follow,

$$
\rho_{a . l}(m+1)=\rho_{a . l}(m)-2 \mu r_{a . \eta\left(m-d_{h y}\right)}\left(z_{m}-v_{m}\right)^{*}
$$

where $d_{b y}$ is the delay value added to compensate for the latency introduced by the feedforward filter (A more detailed explanation of why $d_{b r}$ is needed and how to determine its value is given in Chapter 4). As a result of the sampling rate difference, the beam-former should either update the tap weights every $\beta \chi$ times or reuse the same 
error signals for $\beta \chi$ consecutive updates. (In this thesis, the former approach is chosen because of the ease of implementation.)

\subsection{Discussion}

This chapter has shown a mathematical description of a SIMO digital transmission smart antenna system, which is composed of a transmitter, a SIMO channel, a receiver front-end, and a smart antenna signal processor. Using (24), (27), (29), and (31), a simulation system can be built to study the different beam-former and equalizer architectures. Two different architectures will be investigated here: the traditional beamformer architecture, and the combined beam-former and equalizer architecture. One of the major problems with the traditional beam-former is the limitation of the acquisition range if a MMSE based adaptive algorithm is used. The combined beam-former and equalizer architecture is an attempt to address this problem. However, the combined beam-former and equalizer architecture suffers from a number of problems and these problems, along with some of their solutions, will be shown in Chapter 3 and Chapter 4. 


\section{Adaptive Beam-forming and Equalization Parameter Selection}

Based on the system models developed above, there are a number of system level parameters that impact the performance of the smart antenna receiver system. Particularly for the combined beam-former and equalizer, there are three major system level parameters, which can be adjusted to obtain different levels of performance and computational requirement trade-offs: the length of the beam-former, the length of the forward filter in the equalizer and the down-sampling ratio of the forward filter. The first part of the chapter is dedicated to how to optimize the different system level parameters for the combined beam-former and equalizer system. The effect of each parameter on the convergence time and BER will also be discussed.

After a set of optimal parameters for the combined beam-former and equalizer architecture has been established, the second part of the chapter focuses on the advantages and disadvantages of the combined beam-former and equalizer architecture. The combined beam-former and equalizer architecture has many desirable properties when compared to the traditional beam-former, such as, a wider acquisition range and combining signals from multiple signal paths in a large delay spread channel. However, because the error signal used to update the beam-former is shared with the equalizer, this architecture suffers from a number of problems, sensitivity to the error signal, slow convergence time, and variable error signal feedback delay. 


\subsection{Simulation Model}

There are two signal sources in the system; one is the desired signal and the other is interference; while three signal paths are used for the desired signal, the remaining two paths are used for interference. Not all of the paths are active at the same time. For some of the simulations, the path carrying the interference is de-activated to simulate an interference-free channel environment. For each signal path, the channel is modeled using equation (24) derived in Chapter 2.3. Table I lists all of the channel parameters that are constrained in the simulations.

Table I The following table shows the value for each of the channel parameters.

\begin{tabular}{|l|l|l|}
\hline $\begin{array}{l}\text { Parameter } \\
\text { Name }\end{array}$ & Value & Description \\
\hline$K_{m}$ & 3 & $\begin{array}{l}\text { The number of message signal paths is set at three to } \\
\text { limit computation time. }\end{array}$ \\
\hline$K_{i}$ & $-15 \mathrm{~dB}$ & $\begin{array}{l}\text { The number of interference signal paths is set at two to } \\
\text { limit computation time. }\end{array}$ \\
\hline $2 W N_{0}$ & $\begin{array}{l}\text { The power of the Gaussian noise is limited to maintain } \\
\text { a SNR (transmitted power over receiver noise power) } \\
\text { of } 15 \mathrm{~dB} \text {. When interference is added, the noise power } \\
\text { remains constant while the Signal to Interference and } \\
\text { Noise Ratio (SINR) drops. }\end{array}$ \\
\hline$\left|\tau^{\prime \prime}-\tau^{\prime}\right|$ & $\begin{array}{l}\text { N/A delay spread of the channel delay varies from } \\
\text { simulation to simulation: however, it is always kept at a } \\
\text { maximum of } 3 \text { symbol time. The lower and the upper } \\
\text { bound are arbitrarily set to meet the delay spread } \\
\text { requirement. }\end{array}$ \\
\hline$\alpha_{k}$ & $\begin{array}{l}\text { The amplitude of each signal path is randomly chosen } \\
\text { with El } \alpha k \text { I } 1 . \text { During the simulation, the value of } \alpha_{k} \\
\text { is selected at the beginning of the simulation and it } \\
\text { remains constant throughout the simulation. This } \\
\text { assumption is valid because the channel is slowly } \\
\text { varying. }\end{array}$ \\
\hline
\end{tabular}




\begin{tabular}{|l|l|l|}
\hline $\begin{array}{l}\text { Parameter } \\
\text { Name }\end{array}$ & Value & Description \\
\hline$\theta_{k}$ & N/A & $\begin{array}{l}\text { The angle of arrival for each signal path is arbitrarily } \\
\text { chosen during each simulation, since the angle arrival } \\
\text { doesn't significantly affect the performance of the } \\
\text { system. }\end{array}$ \\
\hline
\end{tabular}

The transmission system used in the simulation is a simple QPSK digital communication system. Because the system is simulated in software, in order to keep the simulation relatively short, both the channel and the transmission system are simulated in baseband. Table 2 lists all of the parameters used to constrain the transmission system.

Table 2 The following table shows the value for each of the transmission system parameters.

\begin{tabular}{|l|l|l|}
\hline $\begin{array}{l}\text { Parameter } \\
\text { Name }\end{array}$ & Value & Description \\
\hline$N_{A}$ & 3 & $\begin{array}{l}\text { The number of antenna is limited to three because the } \\
\text { smart antenna system is able to generate } N_{A}-1 \text { nulls to } \\
\text { cancel the two sources of interference. (The } \\
\text { interference comes from two different signal paths. }\end{array}$ \\
\hline$\eta$ & 8 & $\begin{array}{l}\text { The number of samples per symbol is set to } 8 \text { to limit } \\
\text { the required length of the beam-former and the } \\
\text { equalizer. }\end{array}$ \\
\hline$\beta$ & N/A & $\begin{array}{l}\text { The down-sampling factor, or the fractional step size, } \\
\text { of the matched filter is one of the parameters being } \\
\text { studied. In typical simulations. } \chi \text { is set to 4. }\end{array}$ \\
\hline$T$ & N/A & $\begin{array}{l}\text { The down-sampling rate of the feedforward filter. } \beta, \\
\text { varies from simulation to simulation. However, } \beta \chi \text { is } \\
\text { constrained to } 8 .\end{array}$ \\
\hline$\xi$ & 1 & $\begin{array}{l}\text { The symbol rate of the system is set to 250 ksymbols/s } \\
\text { to mimic the symbol rate of the experimental capture } \\
\text { system described in section 5. }\end{array}$ \\
\hline & $\begin{array}{l}\text { The update rate of the beam-former LMS algorithm is } \\
\text { set to I to improve the convergence characteristics of } \\
\text { the system. }\end{array}$ \\
\hline
\end{tabular}




\begin{tabular}{|c|c|c|}
\hline $\begin{array}{l}\text { Parameter } \\
\text { Name }\end{array}$ & Value & Description \\
\hline$\rho$ & 0.5 & $\begin{array}{l}\text { The distance between the antennas is set to half the } \\
\text { wavelength of the carrier frequency. }\end{array}$ \\
\hline$f_{i}$ & $2.1875 \mathrm{GHz}$ & $\begin{array}{l}\text { The carrier frequency of the system is set to } 2.1875 \\
\mathrm{GHz} \text { to mimic the carrier frequency used in the } \\
\text { experimental capture system described in section } 5 \text {. }\end{array}$ \\
\hline$\tau_{0}$ & 0 & $\begin{array}{l}\text { Since the transmission system is simulated in baseband, } \\
\tau_{0} \text { becomes irrelevant to the system because there is no } \\
\text { frequency lock requirement. }\end{array}$ \\
\hline$\alpha$ & 1 & $\begin{array}{l}\text { Because bandwidth is not an issue in the simulated } \\
\text { environment, the roll of factor of the pulse shape filter, } \\
\alpha \text {, is set at } 1 \text { to improve the SNR. }\end{array}$ \\
\hline$L_{H r}$ & $\mathrm{~N} / \mathrm{A}$ & $\begin{array}{l}\text { The length of the beam-former is one of the parameters } \\
\text { being studied. In typical simulations, } L_{B t} \text { is set to } 2 \\
\text { symbol length, which is } 16 \text {. }\end{array}$ \\
\hline$L_{r t}$ & N/A & $\begin{array}{l}\text { The length of the feedforward filter is one of the } \\
\text { parameters being studied. In typical simulations, } L_{F x} \\
\text { is set to } 48 \text {. }\end{array}$ \\
\hline$L_{H E}$ & 8 & $\begin{array}{l}\text { The length of the feedback filter is currently being } \\
\text { studied; therefore, it is set to an arbitrary value. }\end{array}$ \\
\hline$N$ & N/A & $\begin{array}{l}\text { All of the simulation results presented in this section } \\
\text { are based only on the training sequence. }\end{array}$ \\
\hline
\end{tabular}

\subsection{Evaluation Criteria}

The performance of the system will be judged based on three criteria: bit error rate, convergence time, and computational complexity. The ideal system should be able to satisfy all three requirements. The following section describes how these criteria are measured and some of the assumptions made.

\subsubsection{Bit Error Rate Performance}

The bit error rate measurement is based on following assumptions: 
1. The bit error rate performance of the system depends heavily on the white noise in the system and the interference injected into the system. Therefore, the system will be measured in two different environments. First of all, the noise level remains constant and there is no interference. Secondly, the noise level remains constant while the interference power varies.

2. The delay spread of the system, and the AOA of different signal paths could also affect the bit error performance of the system. Therefore, the bit error rate measurement should be the average performance of at least 5 random channel configurations.

The ideal system should have reasonable BER performance. Many other smart antenna studies have dealt with BER performance of the system, and similar performance gain has been observed using the current simulation setup $|11|$ : therefore, it will be the major focus of this thesis.

\subsubsection{Convergence Time}

Similar to the bit error rate measurements, the convergence time is also based on two basic assumptions:

1. The convergence time of the system is heavily dependent on the overall noise level of the system as well as the interference power in the environment. Therefore, all of the convergence time data should be gathered based on a set noise level and interference power.

2. The convergence time of the system could also be affected by the delay spread as well as the AOA of different signal paths. Consequently, the configuration will 
have a significant impact on the performance of the system. Hence, the convergence time should be averaged over at least 5 simulations with random channel parameters.

The convergence time is usually measured by the number of training symbols required for the weight taps of the system to be optimized. In many systems, a couple of frames of training sequences are sent initially to allow the system to lock on. In this thesis, the system is said to have achieved a locked state when the mean square error (MSE) signal magnitude has reached a steady state; this occurs typically after a couple of hundred symbols of training.

\subsubsection{Computational Efficiency}

The system complexity could be measured as the number of arithmetic operations required to generate a single output value. An addition operation is defined as having a computational complexity of 1 . In hardware, a multiplication could be implemented using an array multiplier. An array multiplier requires $\log _{2}(N)$ addition operations, where $\mathrm{N}$ is the largest number used in the system: therefore, the computation complexity of a multiplication operation is defined as $\log _{2}(N)$. As a result, the system complexity of the LMS beam-former is defined by

$$
C_{\text {berm-former }}=C_{b f}+C_{\text {IMSth }}
$$

where $C_{b f}$ is the computational complexity for generating a single output, which is defined as follows,

$$
C_{b f}=N_{A} \times\left(\log _{2}(N) \times L_{B F}+L_{B F}-1\right)+1
$$


and $C_{\text {LISS }}$ is the computational complexity for updating the tap weights of the beamformer, which is defined as follows,

$$
C_{L M S h f}=N_{A} \times L_{B F} \times\left(2 \times \log _{2}(N)+1\right)+1
$$

A similar equation can be derived for the decision feedback equalizer,

$$
C_{e q}=C_{f f}+C_{f b}+C_{\text {LMSeq }}
$$

where $C_{f f}$ is the computational complexity of the feedforward filter, which is defined as follows,

$$
C_{f f}=\left(\log _{2}(N) \times L_{F F}+L_{F F}-1\right)
$$

and $C_{h f}$ is the computational complexity of the feedback filter, which is defined as follows,

$$
C_{f b}=\left(\log _{2}(N) \times L_{F B}+L_{F B}-1\right)
$$

and $C_{L A M S e q}$ is the computational complexity of the update equation for the equalizer. which is defined as follows,

$$
C_{L M S e q}=\left(L_{F F}+L_{F B}\right) \times\left(2 \times \log _{2}(N)+1\right)
$$

The computational complexity of the system is equal to

$$
C_{s y s}=C_{e q}+C_{b e a m-\text { frrmer }}
$$




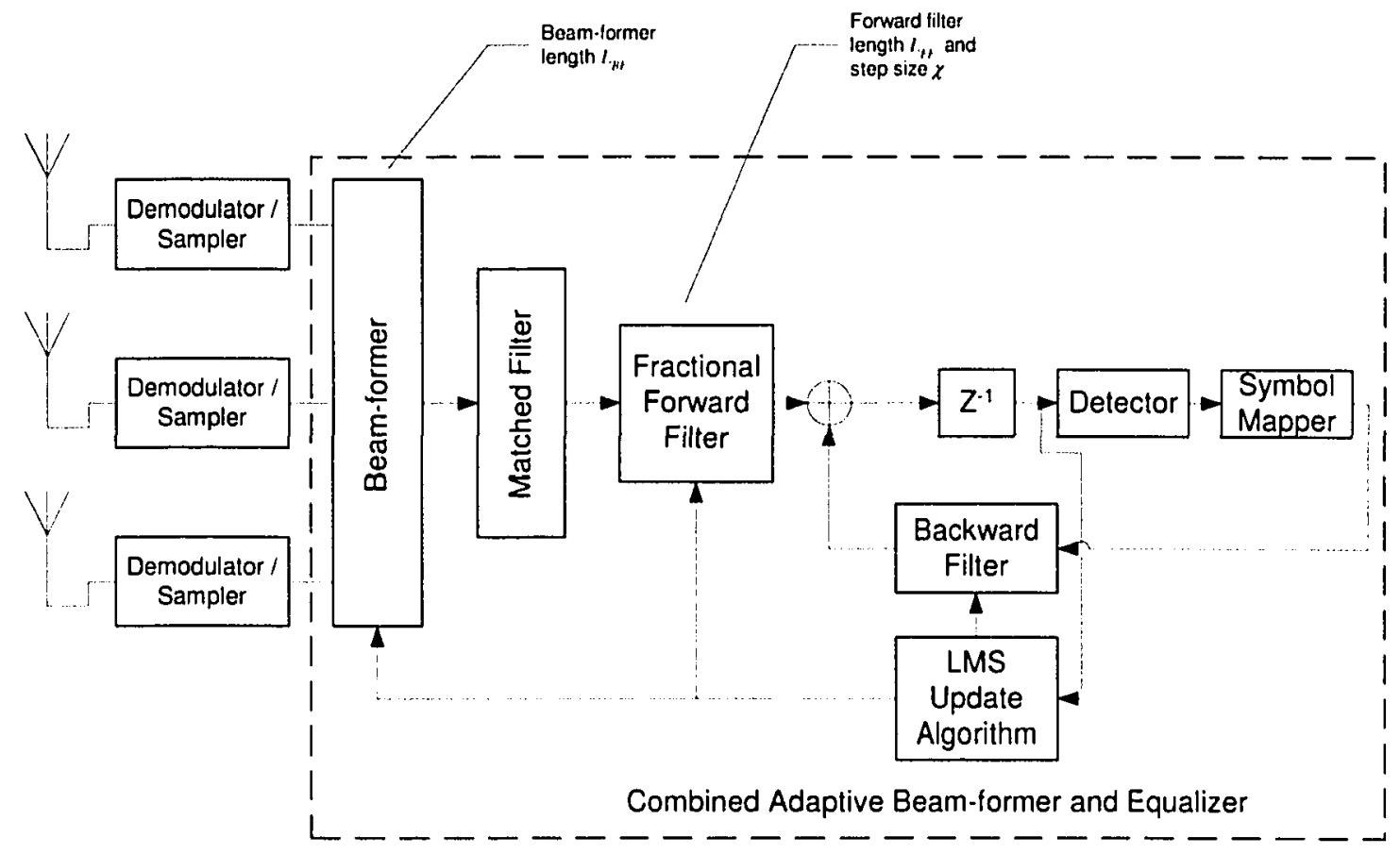

Figure 10. There are three different parameters that can be adjusted in the combined beam-former and equalizer architecture: the length of the beam-former, the down-sampler ratio, and finally, the length of the feedforward filter.

\subsection{Parameter Selections for the Combined beam-former and equalizer}

There are several parameters in the combined beam-former and equalizer design. shown in Figure 10, which need to be carefully selected to optimize the performance of the design, such as, the length of the beam-former $L_{B F}$, the length of the feedforward filter, $L_{r i}$, and the step size, $\chi$, of the fractionally spaced feedforward filter. Some of the parameters have been examined in |11| and |12|: however, those studies focused mainly on the resulting bit error rate of the system and the convergence characteristic was not discussed. Therefore, the following section will examine both how each of the 
parameters will affect the performance of the system and the optimal selection for each of the parameters.

\subsubsection{Beam-former Length}

The length of the beam-former has been extensively studied in $[11]$ and the study shows that, as the delay spread of the signal increases, the system performance can be improved by increasing the length, $L_{B r}$, of the beam-former. In addition, asymptotic behavior of the bit error rate is observed as the number taps increase beyond the delay spread of the channel. However, the study in [11] only focuses on the resulting bit error rate while the convergence characteristic is ignored, i.e., it has not answered the question whether increasing the number taps in the beam-former will increase the convergence speed of the LMS adaptive algorithm.

Using the system model described in Section 2, several simulations have been run with different values of $L_{B F}$. In all the simulations, the equalizer length, $L_{l F}$, is set to 16 and the feedforward filter step ratio, $\chi$, is set to 1 . The proper value for $L_{r+}$ should large in order to reduce the adverse effect of the equalizer on the overall performance of the receiver; the optimized value for $L_{\psi}$. will be discussed in the subsequent section. The delay spread $\left|\tau^{\prime \prime}-\tau^{\prime}\right|$ of the channel is kept within a bound of 1.5 symbols; the amplitude of each signal path, $\alpha_{k}$, and the angle of arrival for each signal path. $\theta_{k}$, are randomly chosen. For each value of $L_{B F}, 5$ random simulations are run: the MSE signals are filtered using a moving average filter with a window size of 25 samples and then the MSE signals from each simulation are averaged to generate the plot shown in Figure 11. 


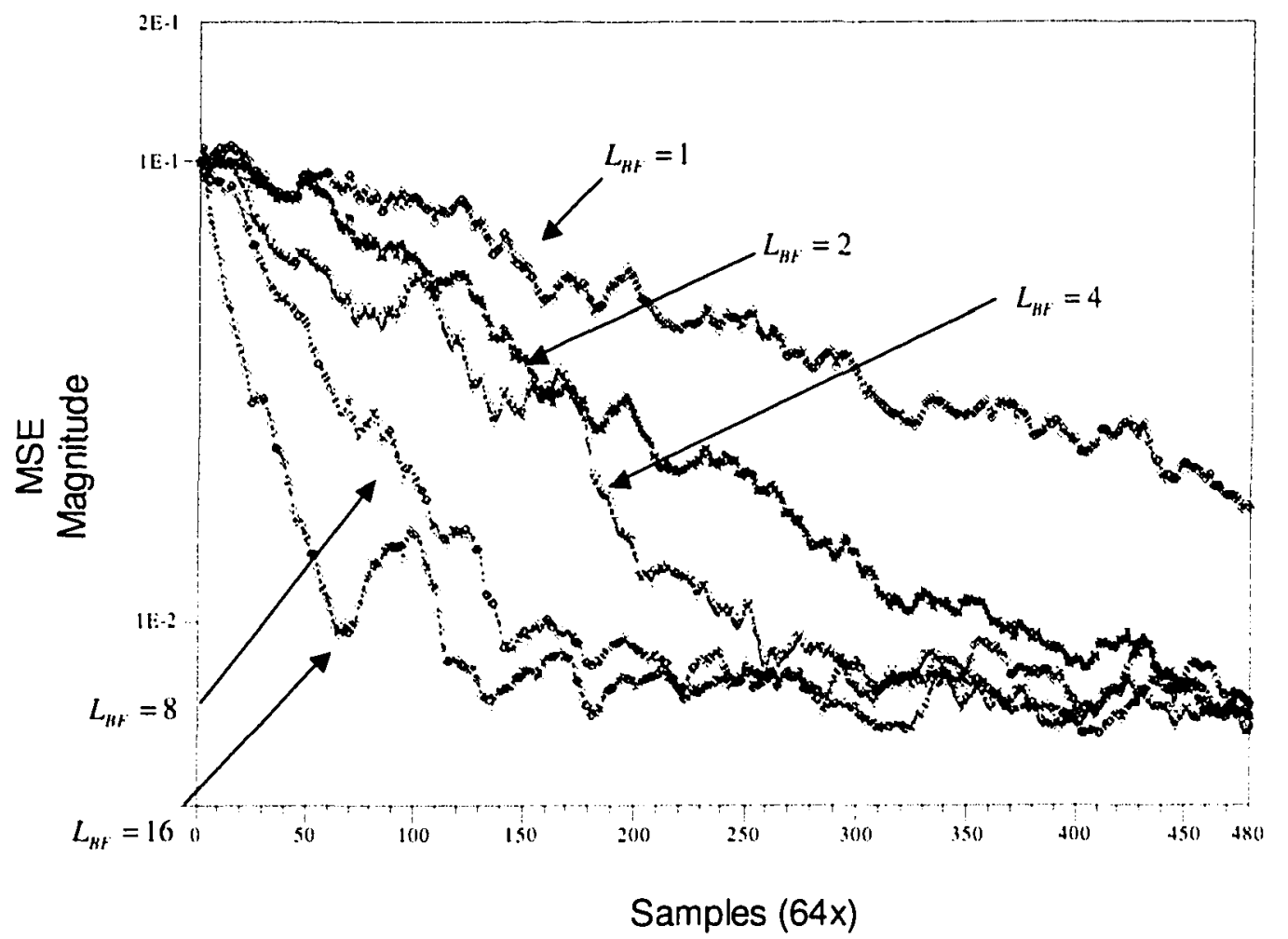

Figure 11. As the value of $L_{k r}$ varies, the MSE declines much more quickly; however, the improvement diminishes as $L_{k r}$ becomes larger.

The plot shows that the speed of convergence increases as the value of $L_{A r}$ increases. However, an additional observation can be made. The final magnitude of the MSE signal seems to be converging at the same level for all values of $L_{H F}$. To further illustrate this behavior, when the value of $L_{H r}$ for the beam-former is set to 16 , the convergence performance for this beam-former does not differ significantly from the beam-former, with $L_{B F}$ set to 8 . Based on this observation, one can assume that the optimal value of $L_{B r}$ is related to the delay spread of the channel. (In all the simulations, the delay spread of the individual path is limited to about one and a half symbols in length. Therefore, as 
the number of taps approaches the 2 symbol length, the improvement diminishes.) For a channel with a high level of delay spread, increasing the value of $L_{B H}$ will the system performance. However, equation (40), (41) and (42) have shown that $C_{\text {leam-furmer }} \propto L_{B F}$; therefore, increasing the value of $L_{B r}$ will increase the computational requirements of the system significantly.

\subsubsection{FSE Equalizer Length}

In the traditional beam-former system, there is an equalizer immediately after the beam-former and the feedforward filter length in $[11]$ is assumed to be 1 . In the system described in [12] and [6], the equalizer length is greater than one but there is only one tap in the beam-former. From the previous section, we observe that as the length of the beam-former increases, the performance of the system will also increase, as long as the delay spread is low. However, the equalizer length $L_{r+}$ can be much longer than that of the beam-former; therefore, it can mimic a channel response with a much larger delay spread.|12| (The same effect can be achieved by increasing the length of the beamformer, $L_{B r}$, but the computational resources required would be far greater than extending the length of the equalizer.)

In order to demonstrate the performance improvement obtained by increasing the equalizer length $L_{i F}$, three simulations are run. In the first simulation. three receiver systems are simulated. For the first receiver system, the beam-former length $L_{k r}$ is set to 8 and the equalizer length $L_{r r}$ is equal to 8 as well, while $\chi$ is set to 2. For the second receiver system, the beam-former length $L_{r H}$ is set to 8 and the equalizer length $L_{r+}$ is set 
to 1 , while $\chi$ is set to 2 . For the third receiver, the beam-former length $L_{B r}$ is set to 1 and the equalizer length $L_{F F}$ is set to 8 , while $\chi$ is set to 2 . Using the system length model described in Chapter 2, the delay spread $\left|\tau^{\prime \prime}-\tau^{\prime}\right|$ of the channel is kept within a bound of 1.5 symbols; the amplitude of each signal path, $\alpha_{k}$, and the angle of arrival for each signal path, $\theta_{k}$, are randomly chosen for each simulation. Again, the results presented in Figure 12 represent the average magnitude of the MSE signal of 5 simulations; the resulting MSE signal is further filtered using a moving average filter with a window size of 25 samples.

As it is clearly indicated in the above graph, the equalizer length $L_{r i}$ plays an important role in the convergence speed of the beam-former. The system with the larger value of $L_{r F}$ converges more quickly than the system with $L_{k r}$ set to one, given that the value of $L_{H r}$ is the same. However, the receiver with $L_{r F}$ set to 8 and $L_{B r}$ set to I under-performs the other receiver systems. This result clearly indicates that relying on the equalizer alone to improve the system performance is not enough. In addition, the resulting MSE is lower for the system with a large value of $L_{1 \%}$. During the setup of the simulations, the system with a smaller value of $L_{r}$ requires careful tuning of the delay of the desired signal in the training sequence. (The LMS algorithm requires that the incoming signal is correlated with the desired signal.) If the tuning is done incorrectly, the system fails to converge. To compare the ability of the different beam-former systems to mitigate interference, another simulation is run using the same setup as described above but with the two interference paths activated. The interference source is assumed to have the same carrier frequency and the same modulation scheme. The only 


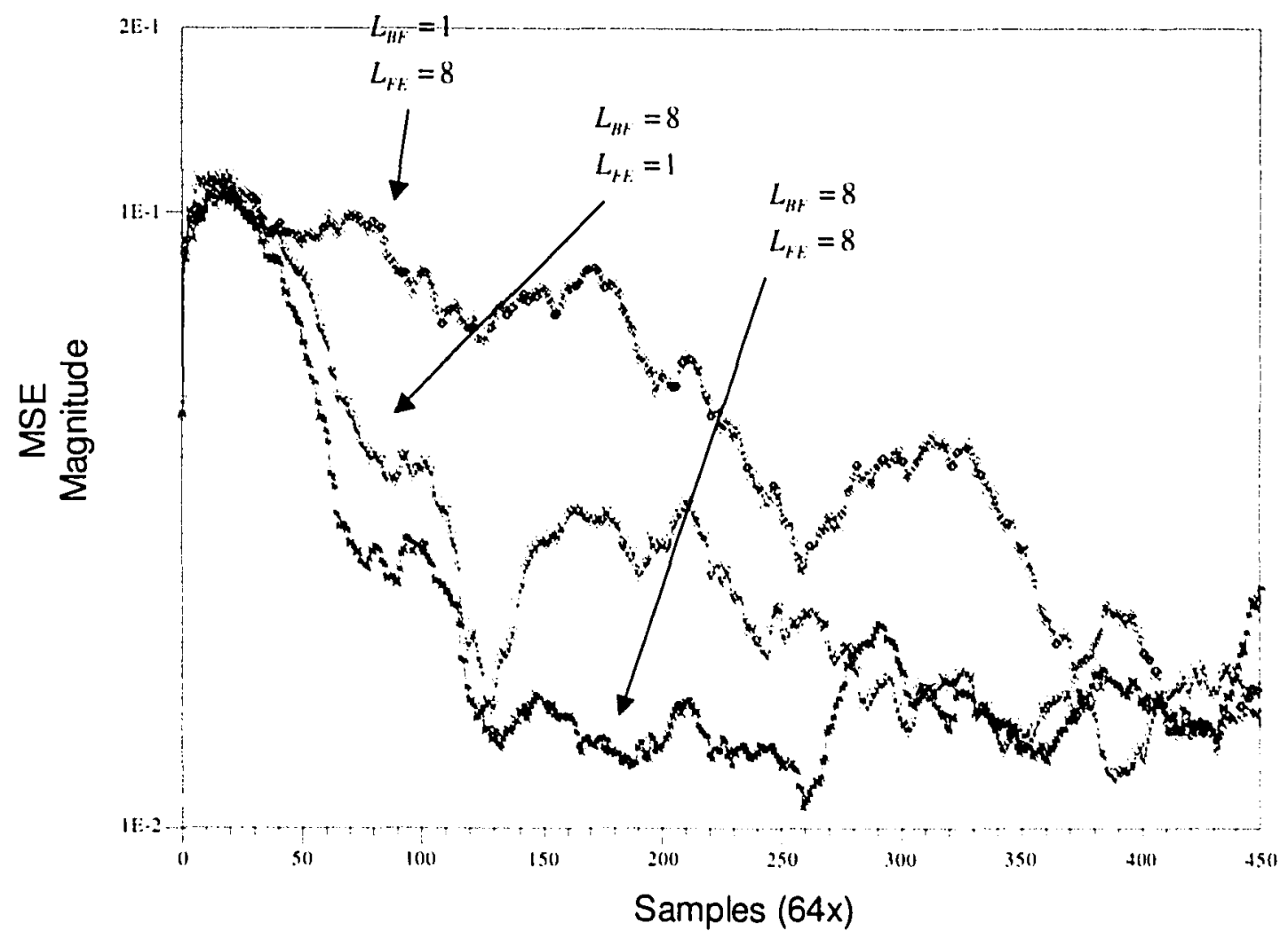

Figure 12. The graph shows the MSE signal going into the beam-former for a different value of $L_{H,}$.

difference between the interferences and the training signal is the data content. Figure 13 has clearly shown that none of the receivers is able to handle interference cancellation well and this is probably due to the improper tuning of the desired signal latency value. Nonetheless, the system with the larger value of $L_{r+}$ performs slightly better than the other systems.

In the third simulation, the same three receivers are used: instead of using a delay spread of only one and a half symbols, a delay spread of 3 symbols is used and the interference is removed. Figure 14 has shown that two out of the three receivers fail to converge to a solution. Although the system performance is still sub-optimal, the 


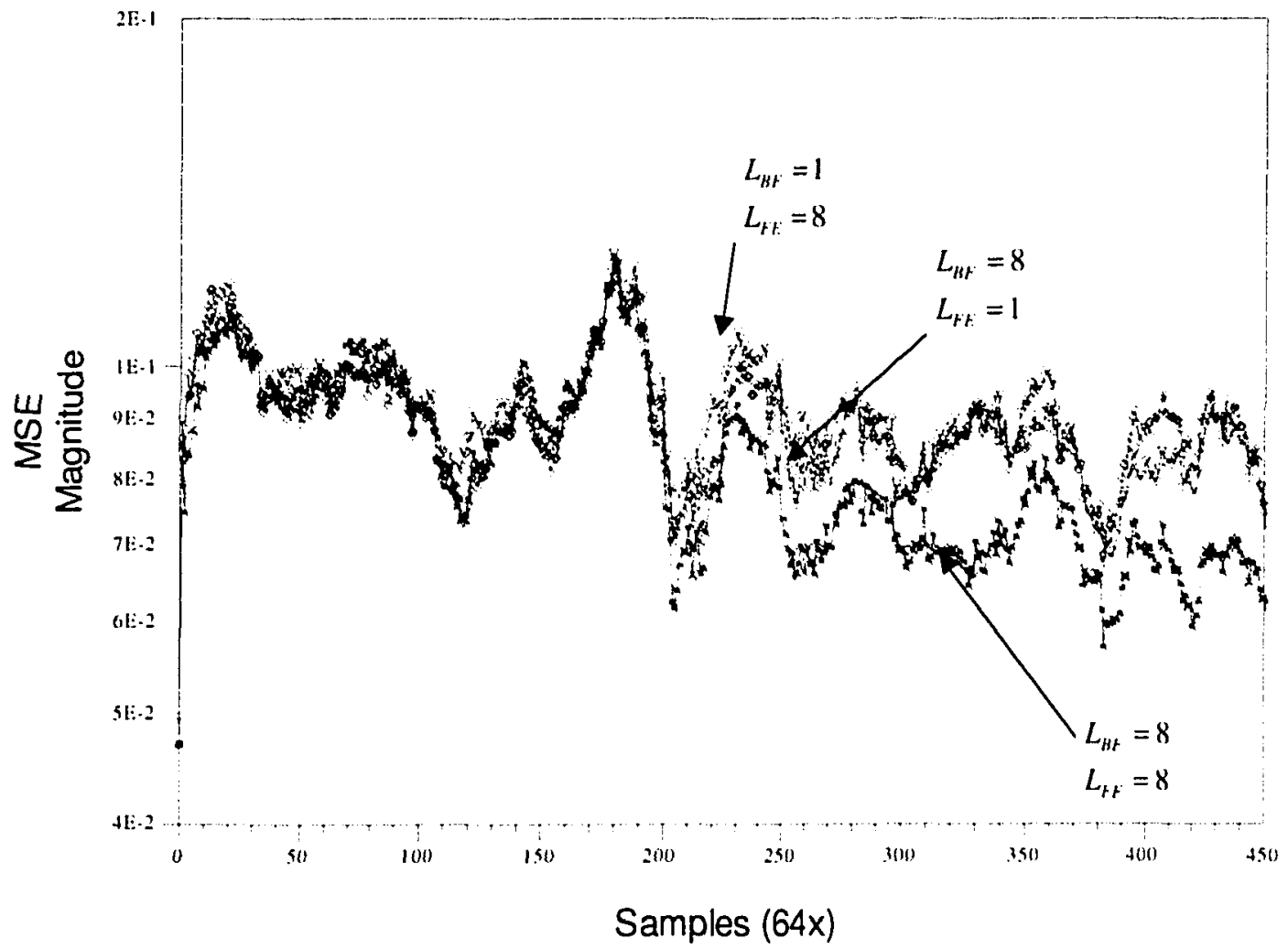

Figure 13. This simulation is run with 2 interlerence sources. Although none of the systems seems to be able to converge to a solution, the systems with a higher value of $L_{k r}$ seem to perform slightly better than systems with a lower value of $L_{r k}$.

receiver with the higher value of both $L_{H F}$ and $L_{H F}$ manages to converge to a solution. This result has clearly shown that having a higher value of $L_{t r}$ will ultimately allow the system to handle channels with larger delay spreads.

\subsubsection{Fractionally Spaced Forward Filter Step Size}

In $|12|$, different fractionally spaced forward filter step size is simulated. It is shown that the as the step size, $\chi$, decreases, the bit error performance improves. Again the study is focused on the bit error, while the convergence characteristic using the LMS 


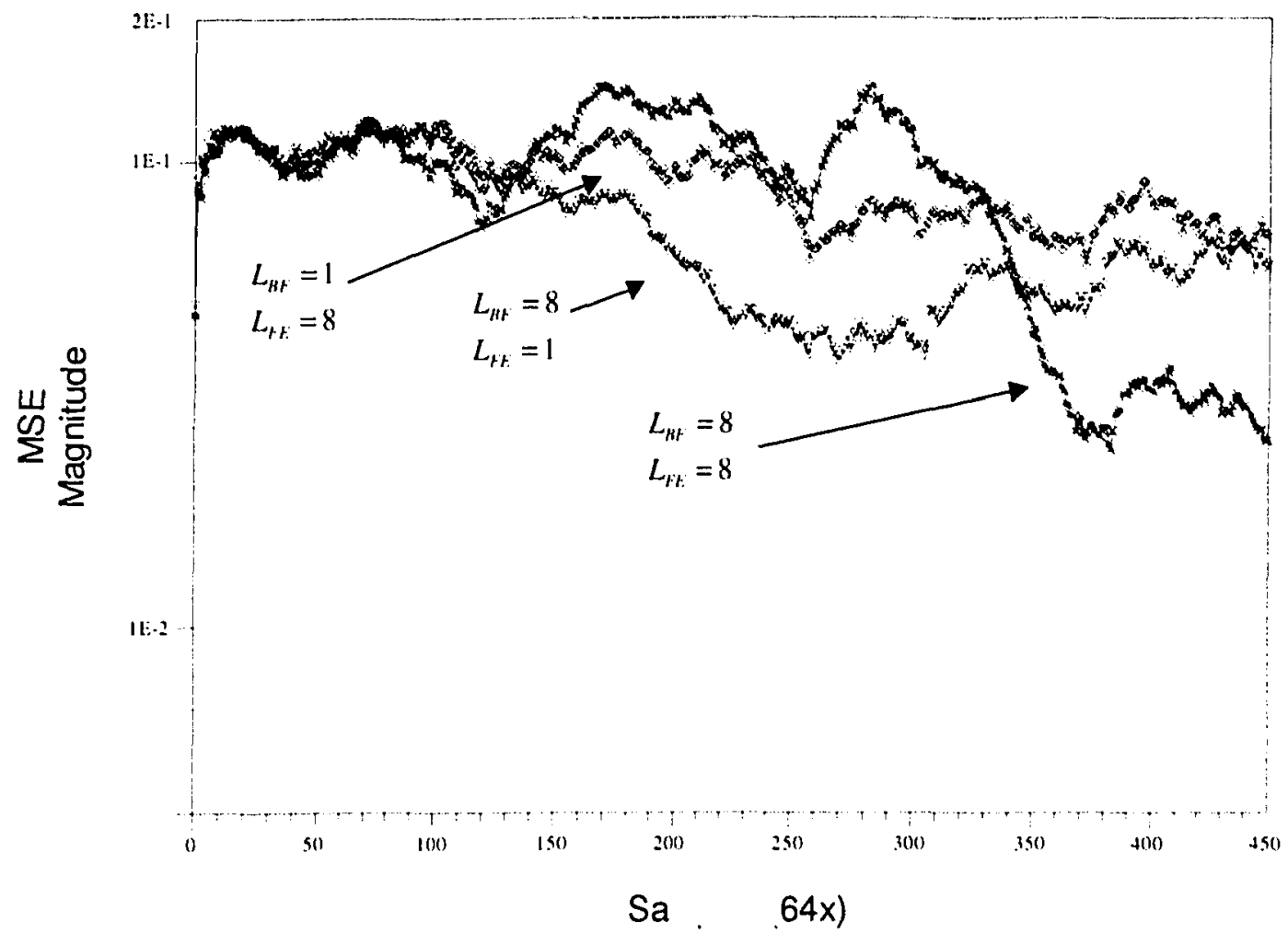

Figure 14. Based on the plot above, one can conclude that the system with the larger value of $L_{k r}$ tends to converge faster and the magnitude of the steady-state MSE signal is lower.

algorithm is not discussed. In addition, the beam-former used in [12] has an $L_{B F}$ equal to 1. As shown in the previous sections, a system with $L_{R r}$ larger than I would improve the overall performance of the system.

To demonstrate the performance gain both in terms of bit error rate and convergence speed as a result of different $\chi$ ratios, several simulations have been run. In the last simulation, a single receiver is modeled with $L_{H r}$ equal to 8 and $L_{r r}$ equal to 32 , while the fractional step size, $\chi$, varies from 1 to 8 . The channel model used in this set of simulations is the same as the one discussed before and the delay spread, $\left|\tau^{\prime \prime}-\tau^{\prime}\right|$, of the 


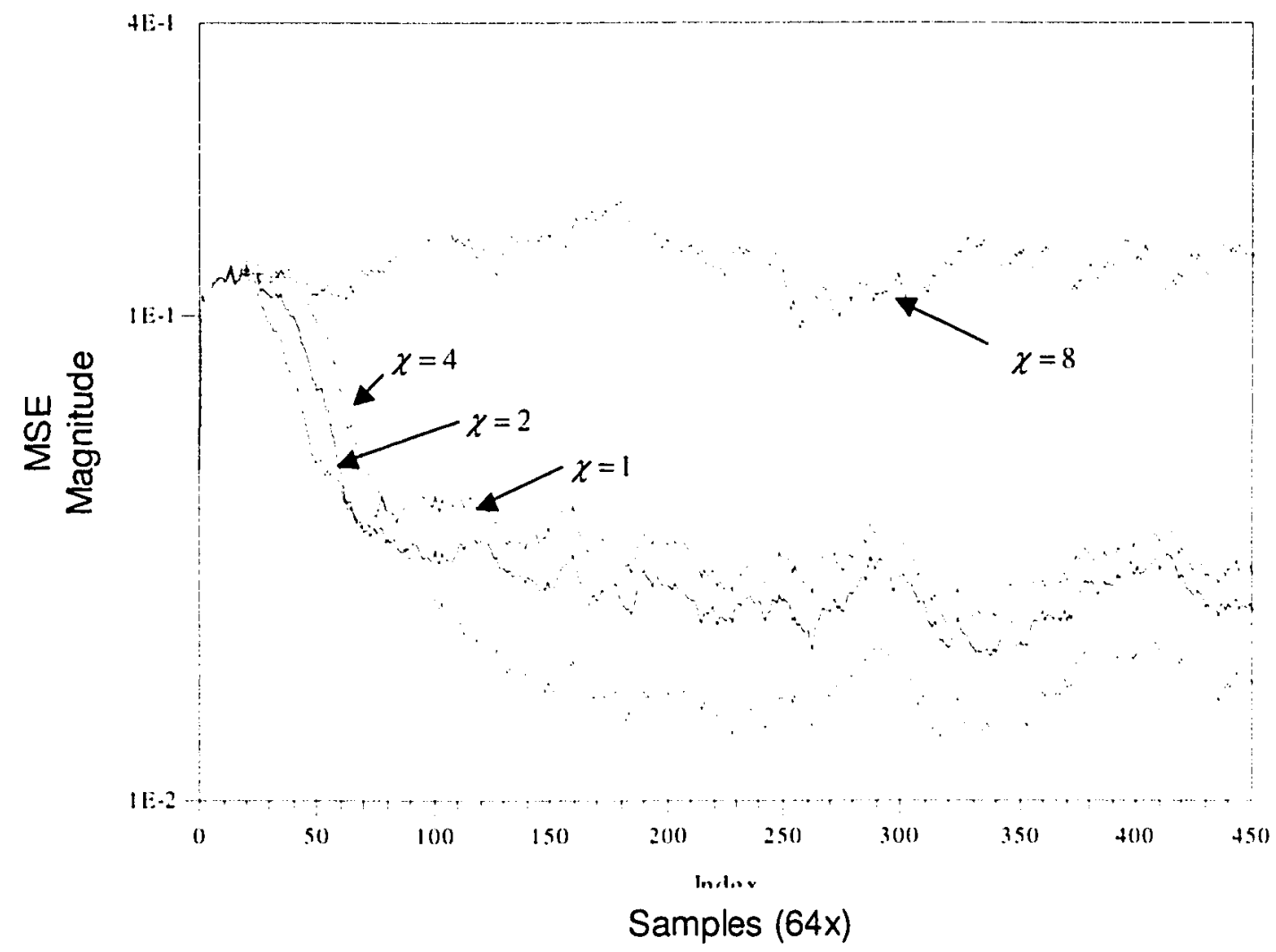

Figure 15. The graph shows the magnitude MSE signal of the receivers with different down sampling ratio, $\chi$. As the ratio decreases, there is marginal improve for the convergence performance.

channel is kept within 1.5 symbols; the amplitude of each signal paths, $\alpha_{k}$, and the angle of arrival for each signal path, $\theta_{\downarrow}$, are randomly chosen for each simulation.

Figure 15 presents the resulting magnitude of the MSE signal over time. The MSE signal shown is an average of 5 simulations and is filtered using a moving average filter with a window size of 25 samples. Figure 15 has indicated that there is some slight improvement in terms of convergence speed and the resulting MSE error as the $\chi$ increases. (When $\chi$ ratio is equal to 8 , the delay of the training sequence is not tuned 


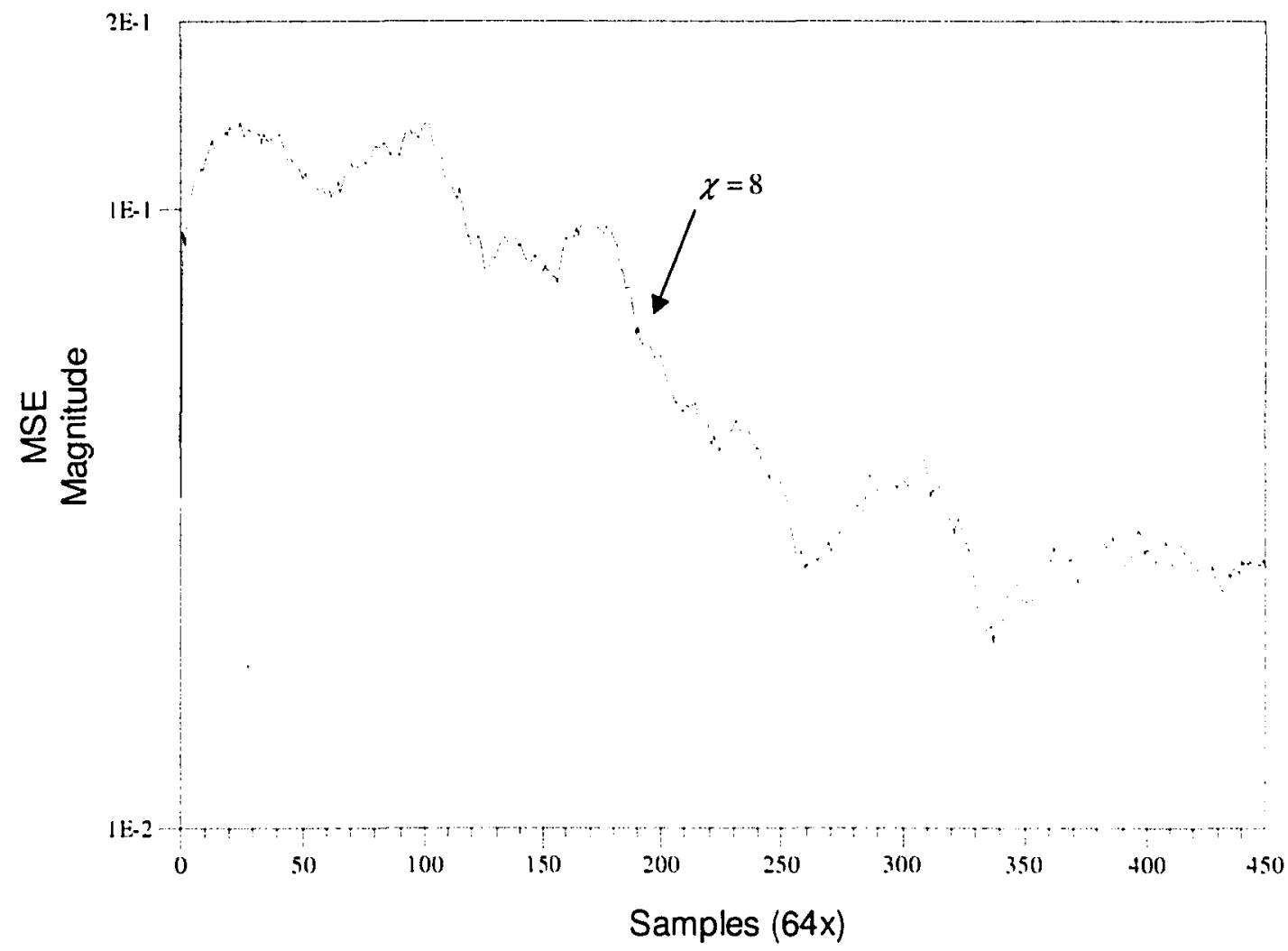

Figure 16. The graph shows the magnitude of the MSE signal of the receivers with the down-sampling ratio, $\chi$, set to 8 . Unlike the graph shown previously, the delay of the training has been tuned; as a result, the system is able to converge. Further tuning can improve the converge speed.

properly; therefore, the simulation fails to converge. Figure 16 shows a properly tuned simulation with $\chi$ ratio equal to 8.) However, the improvement itself is marginal.

\subsubsection{Ideal Beam-former Parameters}

For the LMS based adaptive beam-former, there are three basic parameters which can be optimized, $L_{H F}, L_{H F}$, and $\chi$, which are the length of the beam-former, the length of the feedforward filter and the down-sampling factor of the feedforward filter, 
respectively. Based on the simulations presented above, they indicate that giving each of the parameters larger values seems to improve the performance of the receiver. However, higher resolution and more taps will incur significant computational costs to the receiver system.

For the parameter $L_{k F}$, if one were to observe Figure 11 carefully, as $L_{H F}$ approaches the number of samples per symbol, the improvement of the convergence time begins to diminish. In addition, by examining the result shown in Figure 12 and Figure 14, for large delay spreads, the parameter $L_{F t}$ ultimately determines the overall performance of the receiver system, while the other parameters remain the same. Because it is much more computationally efficient to increase the value of parameter $L_{k F}$, parameter $L_{k F}$ should be kept to around 2 symbols worth of taps.

Parameter $L_{k t}$ determines the number of taps in the equalizer. The principle of the equalizer is to mimic the inverse function of the channel. Consequently, parameter $L_{R T}$ will be heavily dependent on the impulse response of the channel, and the smallest possible value of $L_{r, r}$ should be used to reduce the computational requirements of the receiver.

The ratio, $\chi$, determines the resolution of the equalizer. The computational requirement is proportional to $\chi$ if the ratio $L_{r+} / \chi$ is to remain constant. In Figure 15 , it is very clear that as $\chi$ increases, the convergence time of the system will improve. However, if $\chi$ increases and the number of taps in the equalizer remain unchanged, the performance of the system can become degraded. This is the case when $\chi$ reaches 8 . Consequently, in order to maintain the same level of performance, the equalizer would 
need to be lengthened to match that of the channel. Again, the parameter $L_{\%}$ becomes the dominating factor when computational resources are limited. Therefore, to maximize the performance of the receiver system, one should first of all make parameter $L_{F F}$ match the impulse response of the channel, above all other parameters.

\subsection{Properties of the Combined beam-former and equalizer}

The combined beam-former and equalizer has several benefits over the traditional beam-former. One of the major advantages of the combined beam-former and equalizer is that it has a wider acquisition range; as a result, the beam-former system is able to capture the desired signal without excessive tuning of the training sequence to match the latency of the channel. Secondly, the combined architecture is able to combine signals from different paths with large delay spreads. The following section illustrates both of these advantages.

\subsubsection{Wider Acquisition Range}

Because the combined beam-former and equalizer system feeds the error signal from the equalizer to the beam-former, the system is able to acquire signals, even if the channel has a large delay spread. The feedforward filter length is typically longer than the beam-former; therefore, the equalizer contains a longer time span of signal. Consequently, there are more delayed versions of the transmitted signal in the memory of the equalizer, which makes it easier for the desired signal to be correlated with a copy of the transmitted signal. Although the same effect can be achieved by lengthening the beam-former, it is much more costly computationally, as discussed in Chapter 0. 
A simulation is run to illustrate that, indeed, the combined beam-former and equalizer system has a better acquisition range than the traditional beam-former. Both systems have the same computational complexity and are set up in the same manner, where $L_{B F}$ is set to $16, L_{F F}$ is set to 32 and $\chi$ is set to 2 . The channel model used is the same as the one described in Chapter 3.3.1; the training signal travels to the receiver via three signal paths and there is no interferer in the system. The shortest path is two symbols time longer than the other two paths and its signal strength is made deliberately stronger by $3 \mathrm{db}$. In the simulation, once the two systems reach a steady state, the shortest path is removed. Figure 17 shows the results of the simulation, in which one can see that the system, which uses the combined beam-former and equalizer design. is able to recover from the removal of the line-of-sight signal. The other system simply fails to respond to the change in the channel environment. This is because the equalizer has more taps in the filter, which allows the beam-former to have a wider acquisition range. The separated beam-former and equalizer system will work just as well if either more taps are used in the beam-former or the latency of the desired signal, training sequences. is adjusted to match that of the channel. 


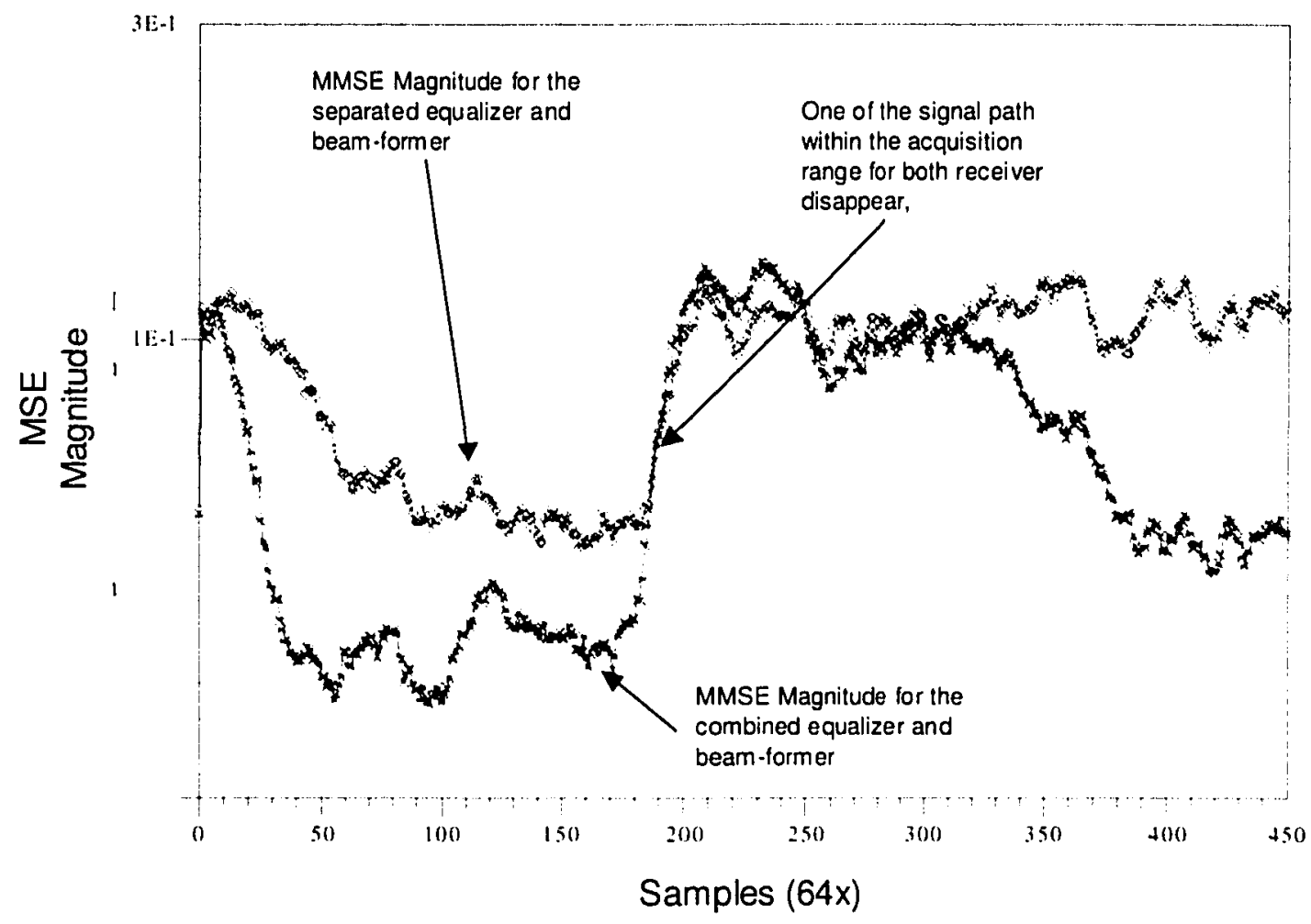

Figure 17. The graph shows the MSE signal going into the two different beam-formers. The combined beam-former and equalizer architecture is able to re-adapt to the loss of the line of sight, while the traditional beam-former architecture fails to compensate.

\subsubsection{Combining Signals from Multiple Paths}

Because the acquisition range of the combined architecture is much larger than that of the original design, it is possible for the entire system to tune to a multiple delayed version of the desired signal. In addition, it is possible for the traditional architecture to lock onto the weakest version of the desired signal (the stronger version of the signal could simply be beyond the acquisition range of the system); hence, the performance of the system would be reduced. In contrast, the large acquisition range gives the combined 


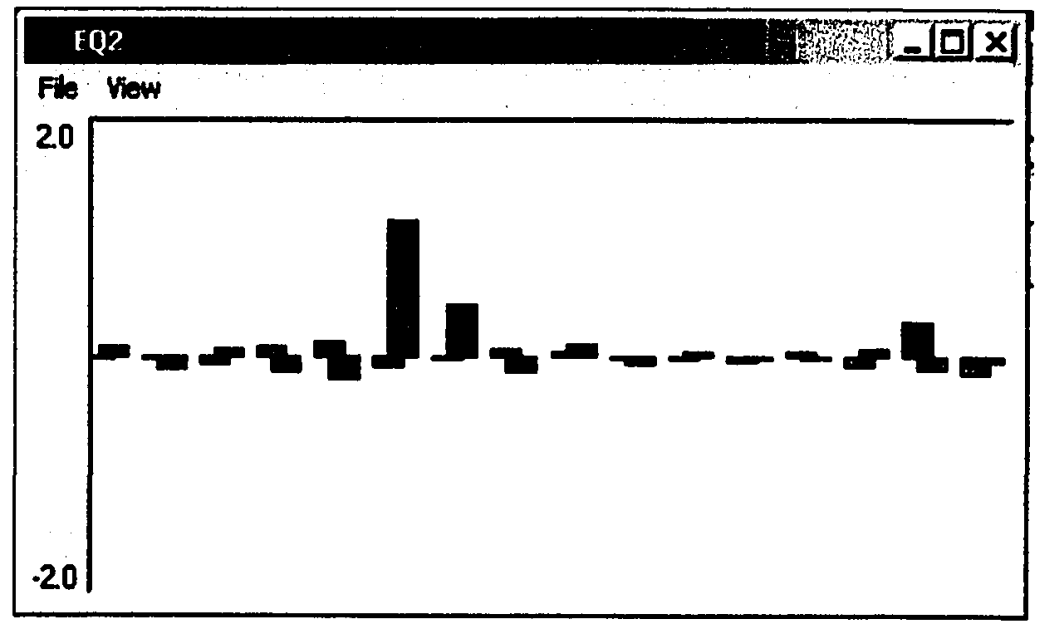

Figure 18. The diagram above shows the magnitude of the taps of the equalizer after the receiver system has entered a steady state.

architecture a better chance to home in on the stronger version of the desired signal. Using the same simulation setup described in the previous section, the tap value of the equalizer is captured when the equalizer has entered a steady state. It is clear from Figure 18 that there are two distinctive non-zero taps in the equalizer, which shows that the equalizer is attempting to combine signals from multiple signal paths.

\subsection{Problems with Combined beam-former and equalizer}

Although the combined beam-former and equalizer architecture can improve the performance of smart antenna systems, it has some undesirable attributes. The following section describes three of the problems suffered by the combined beam-former and equalizer architecture: over-sensitivity to the magnitude of the error signal, slow convergence of the beam-former and variable error feedback latency. 


\subsubsection{Sensitivity to the Magnitude of the Error Signal}

The LMS algorithm can easily become unstable when there is too much noise or interference in the received signal, which causes the error signal to become too large. In the case of the combined beam-former and equalizer architecture, the error signal is no longer strictly the function of the weights in the beam-former, the input signals, and the desired signal. The behavior of the equalizer can have a dramatic effect on the magnitude of the error signal. In addition, the error feedback loop delay is much larger for the beam-former than the equalizer; therefore, the equalizer can react to the change of the signal much more quickly than the beam-former. These two factors together can cause the magnitude of the error signal to remain high for a long period of time: thus, it can cause the beam-former to become unstable. To prevent the adaptive beam-former from becoming unstable, the step size, $\mu$, of the LMS algorithm has to be small.

\subsubsection{Slow Convergence Time}

Because the error signal is affected by the outputs of both the beam-former and the equalizer, changes of the weight taps in either block will perturb the other block. In addition, the error signal loop time for the beam-former is much longer than in the traditional architecture. Consequently, it will take the system longer to converge to a stable solution. Figure 19 is generated by running a number of simulations with varying levels of SNR. By examining the slope of the decreasing MSE signal, one can observe that the traditional beam-former converges faster than the combined architecture when the latency of the desired value is perfectly tuned for the channel. If the desired signal latency does not match that of the channel, the traditional beam-former architecture might not converge to a solution at all. The speed of convergence for the combined architecture 


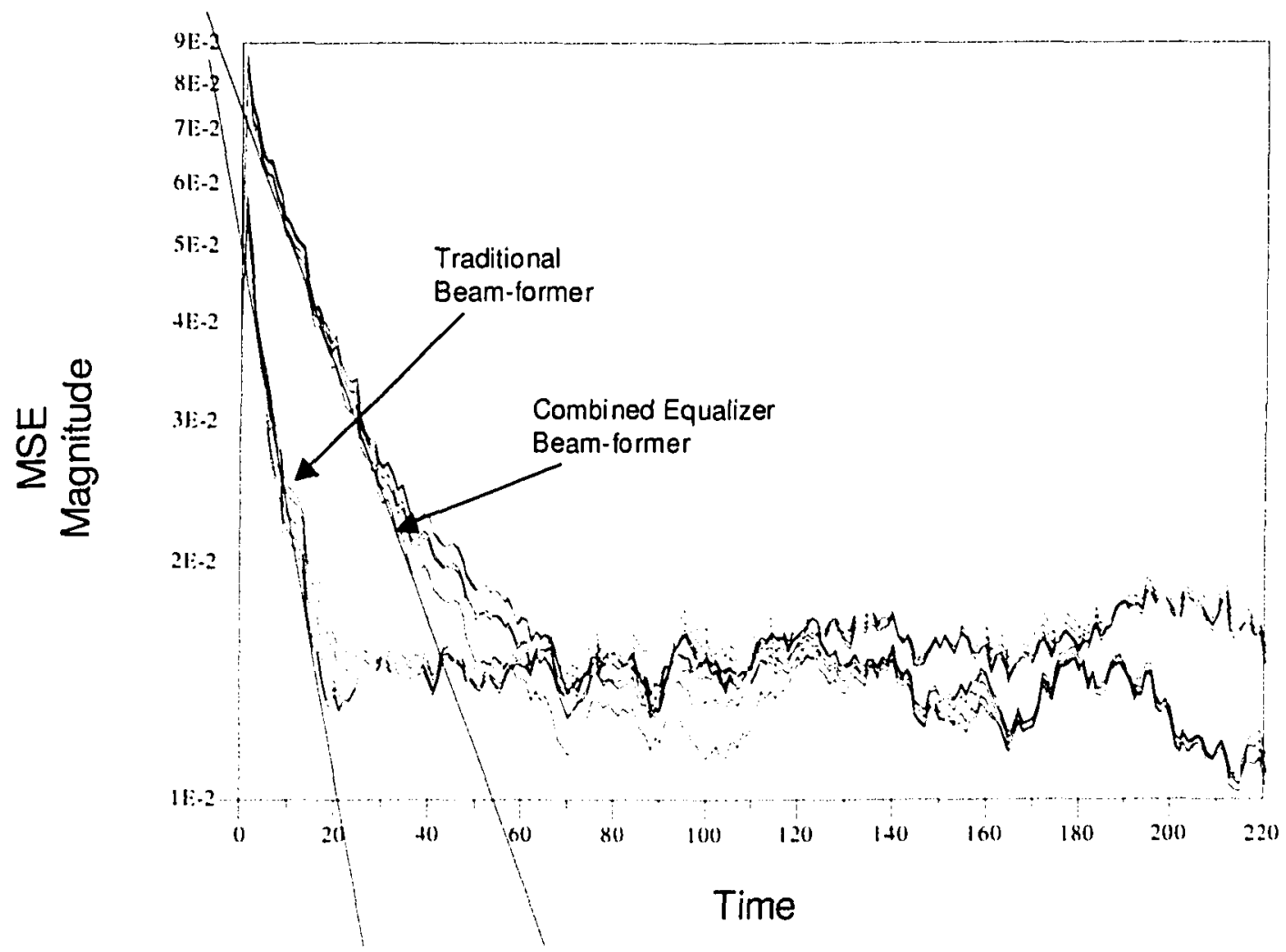

Figure 19. By comparing the magnitude of MSE over time, it is shown from the above graph that the convergence time for the traditional LMS beam-former is faster than that of the combined beam-former and equalizer

will be far better than the traditional beam-former architecture if the latency of the desired signal is less than perfectly tuned.

\subsubsection{Variable Error Signal Feedback Delay}

The variable error signal feedback delay problem is caused by the fact that the feedforward filter path can introduce variable amount delay to the error signal. Hence, the total loop delay of the error signal is not known until the LMS algorithm for the feedforward filter has entered a steady state; moreover, the latency value can vary as the channel changes. 


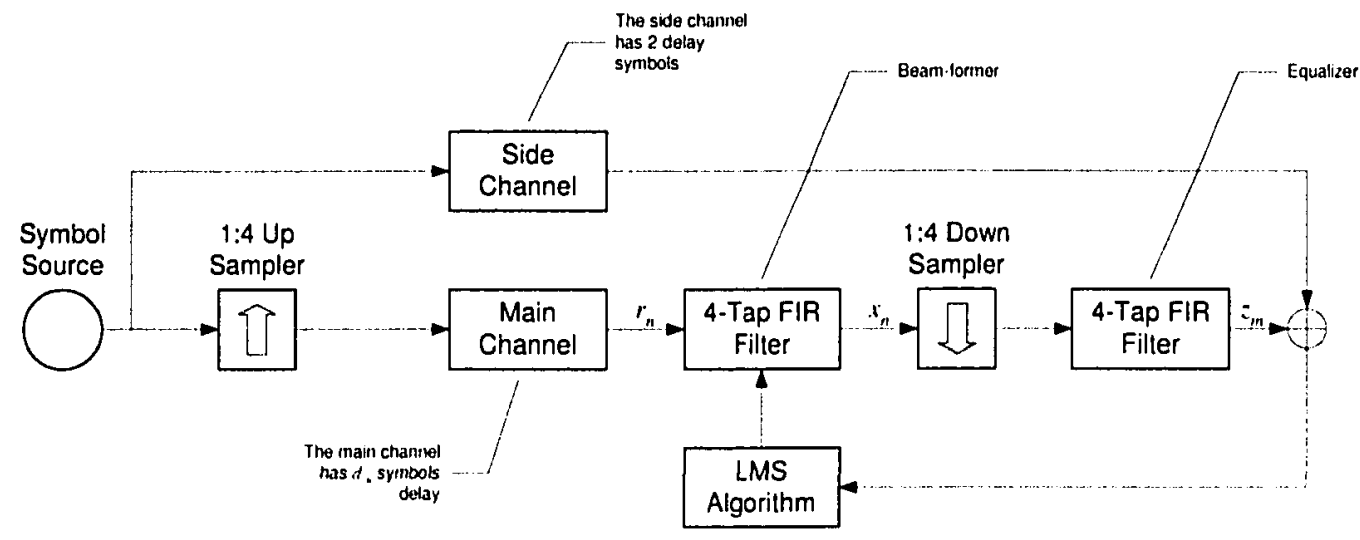

Figure 20. This is a simplified version of the digital transmission system, with only a simple delay channel, a single FIR filter beamformer, and a simple equalizer.

To develop a better understanding of the underlining problem, one can look at a simplified version of a transmission system, as shown in Figure 20. In the figure, a signal source generates a series of symbols, which are then up-sampled 4 times before they are transmitted to the receiver through the channel. The channel is modeled as a simple fixed delay element with delay equal to $d_{t h}$. The same set of symbols is transmitted to the receiver via a side-band channel, which has a fixed delay of $d_{s}=2$, and introduces zero distortion; the signal received via this side-band channel will be used as the desired signal. In the receiver, both the beam-former and the equalizer are represented as simple 4-tap FIR filters. In between the filters, the signals are down-sampled by a ratio of 1 to 4 , $\eta=4$. Assuming the LMS is used to update the weight in both the beam-former and the equalizer; the update equation derived in (39) is still valid.

In the first scenario, the channel delay, $d_{c h}=0 ;$ therefore, when the equalizer enters a steady state, its tap value should be 


$$
\left\{h_{3}, h_{2}, h_{1}, h_{0}\right\}=\{0,0,1,0\}
$$

because the equalizer should delay the symbol by two symbol times in order to match up with the delay of the side-band channel to achieve zero MSE. The table below shows the contents of the beam-former and the equalizer for each sample time.

Table 3 The following table shows the value for each of the system variables at different times, where $d_{c h}$ is equal to 0 .

\begin{tabular}{|l|l|l|l|l|}
\hline Sample Time & $\begin{array}{l}\text { Beam-former } \\
\text { samples } \\
\left\{b_{3}, b_{2}, b_{1}, b_{0}\right\}\end{array}$ & $\begin{array}{l}\text { Equalizer tap } \\
\text { weights } \\
\left\{h_{3}, h_{2}, h_{1}, h_{0}\right\}\end{array}$ & $\begin{array}{l}\text { Equalizer } \\
\text { samples } \\
\left\{a_{3}, a_{2}, a_{1}, a_{0}\right\}\end{array}$ & $\begin{array}{l}\text { Equalizer } \\
\text { Output } z_{m}\end{array}$ \\
\hline 0 & $\left\{r_{0}, 0,0,0\right\}$ & $\{0,0,1,0\}$ & $\left\{x_{0}, 0,0,0\right\}$ & $z_{0}=0$ \\
\hline 4 & $\left\{r_{4}, r_{3}, r_{2}, r_{1}\right\}$ & $\{0,0,1,0\}$ & $\left\{x_{4}, x_{0}, 0,0\right\}$ & $z_{1}=0$ \\
\hline 12 & $\left\{r_{8}, r_{7}, r_{6}, r_{5}\right\}$ & $\{0,0,1,0\}$ & $\left\{x_{8}, x_{4}, x_{10}, 0\right\}$ & $z_{2}=x_{0}$ \\
\hline 16 & $\left\{r_{12}, r_{11}, r_{10}, r_{10}\right\}$ & $\{0,0,1,0\}$ & $\left\{x_{12}, x_{8}, x_{4}, x_{0}\right\}$ & $z_{3}=x_{4}$ \\
\hline & $\left\{r_{16}, r_{15}, r_{14}, r_{13}\right\}$ & $\{0,0,1,0\}$ & $\left\{x_{16}, x_{12}, x_{8}, x_{4}\right\}$ & $z_{4}=x_{8}$ \\
\hline
\end{tabular}

In Table 3, at sample time zero, the output of the beam-former is $x_{0}$ and $x_{10}$ comes out of the equalizer, $z_{2}=x_{0}$, at sample time eight, which is equal to symbol time two, $m=2$. In order for (39) to hold and the LMS algorithm to work,

$$
r_{a, \eta m-1}=r_{8-1}
$$


and

$$
z_{m+d d_{l j}}=z_{2+d d_{b j}}=x_{8}
$$

because if the error signal is not correlated with the input signal, the LMS algorithm will fail. Therefore, based on Table 3, one can conclude that $d_{b j}$ must be equal to 2 in order for the above condition to hold.

If the delay of the channel, $d_{c h}$, is changed from 0 to 1 , the taps in the equalizer will also change, as well. Since the channel has delayed the samples by one symbol time, the equalizer will only need to delay the samples by one symbol time in order for the signal arriving at the sideband channel to match that received from the main channel. Using the new channel condition, a new table is computed.

Table 4 The following table shows the values for each of the system variables at different times, where $d_{c h}$ is equal to 1.

\begin{tabular}{|l|l|l|l|l|}
\hline Sample Times & $\begin{array}{l}\text { Beam-former } \\
\text { samples } \\
\left\{b_{3}, b_{2}, b_{1}, b_{0}\right\}\end{array}$ & $\begin{array}{l}\text { Equalizer tap } \\
\text { weights } \\
\left\{h_{3}, h_{2}, h_{1}, h_{0}\right\}\end{array}$ & $\begin{array}{l}\text { Equalizer } \\
\text { samples } \\
\left\{a_{3}, a_{2}, a_{1}, a_{0}\right\}\end{array}$ & $\begin{array}{l}\text { Equalizer } \\
\text { Output } z_{m}\end{array}$ \\
\hline 0 & $\left\{r_{0}, 0,0,0\right\}$ & $\{0,1,0,0\}$ & $\left\{x_{0}, 0,0,0\right\}$ & $z_{0}=0$ \\
\hline 4 & $\left\{r_{4}, r_{3}, r_{2}, r_{1}\right\}$ & $\{0,1,0,0\}$ & $\left\{x_{4}, x_{0}, 0,0\right\}$ & $z_{1}=x_{0}$ \\
\hline 12 & $\left\{r_{8}, r_{7}, r_{6}, r_{5}\right\}$ & $\{0,1,0,0\}$ & $\left\{x_{8}, x_{4}, x_{0}, 0\right\}$ & $z_{2}=x_{4}$ \\
\hline & $\left\{r_{12}, r_{11}, r_{10}, r_{5}\right\}$ & $\{0,1,0,0\}$ & $\left\{x_{12}, x_{8}, x_{4}, x_{0}\right\}$ & $z_{3}=x_{8}$ \\
\hline
\end{tabular}


In the above table, one can observe that $x_{0}$ comes out of the equalizer one symbol time earlier, $z_{1}=x_{0}$, where $m=1$. As a result, in order to satisfy the condition for (39), $d_{h f} \quad$ ust be equal to 1 .

From the above example, one can easily observe that parameter $d_{h f}$ is heavily dependent on the values of the coefficients in the feedforward filter of the equalizer, which, in turn, depends on the channel delay as well as the delay of the desired signal. As a result, parameter $d_{h j}$ will need to be updated dynamically as the channel condition changes.

To further illustrate this concept, a simulation is run with two antennas and a single signal source. The transmitted signal arrives at the receiver via 2 different signal paths. Parameter, $d_{h f}$, is then swept, and the resulting bit error rate is measured. Figure 21 shows that there exists an optimal error signal feedback delay when $d_{b j}$ is equal to 15 , such that the system performance is maximized, which is due to the shorter line of the sight path. Moreover, there is a local minimum when $d_{b j}$ is equal to 11 . This is due to the weaker paths, which have a higher path latency than the line-of-sight path. 


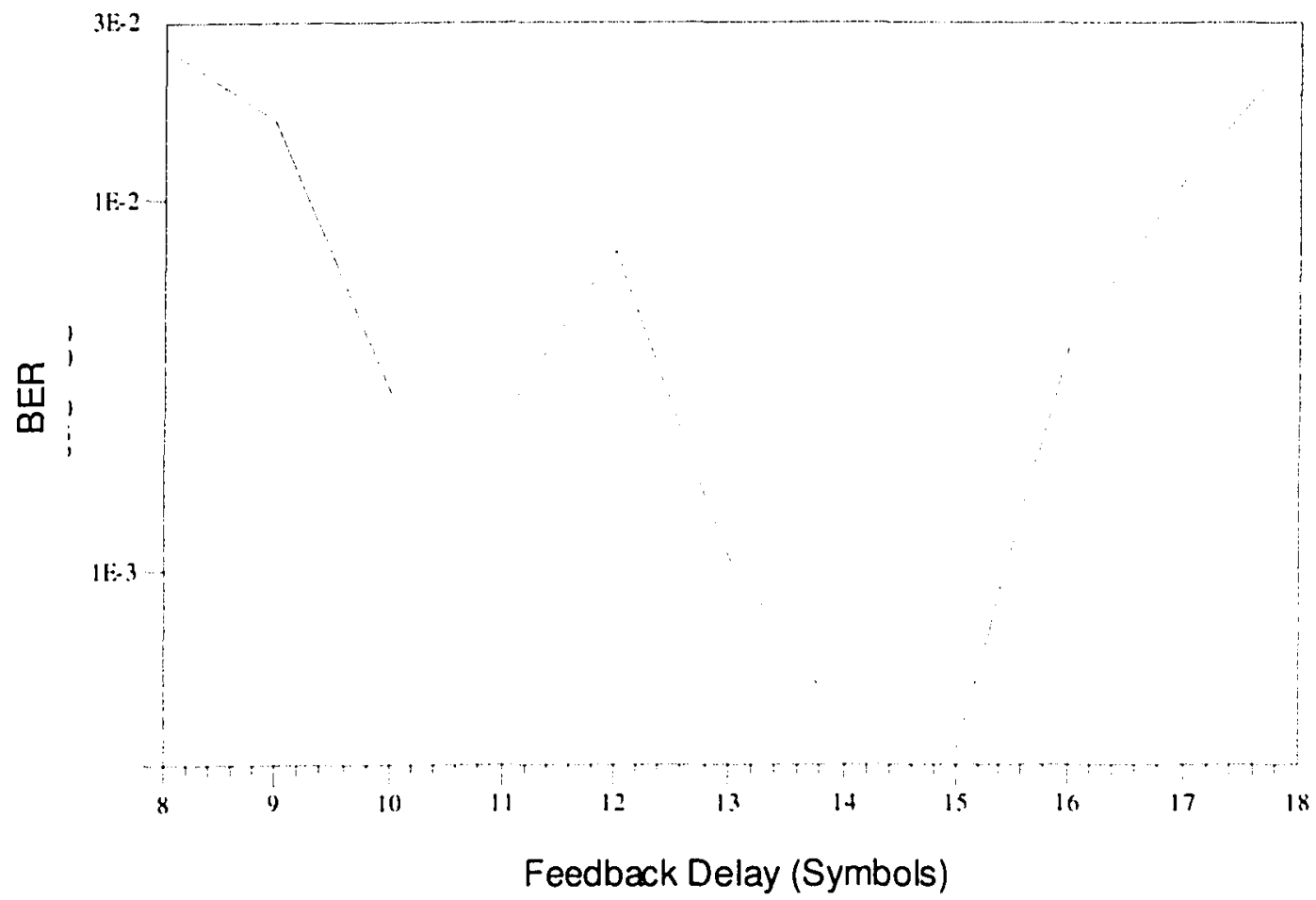

Figure 21. This graph shows the BER of the system as the error signal feedback delay varies.

\subsection{Discussion and Conclusion}

In Chapter 3, a number of system-level parameters, the length of the beamformer, the length of the forward filter of the equalizer, and the down-sampling ratio of the forward filter of the equalizer in the combined beam-former and equalizer architecture are optimized. Using the mathematical model developed in Chapter 2, a number of simulations have been run with various combinations of the parameters stated above. The simulations have shown that increasing the length of the beam-former, the length of the forward filter of the equalizer, and the down-sampling ratio will improve the convergence time of the system. However, the improvement is asymptotic as each of the parameters increases. In addition, the computing resource requirement for increasing the 
length of the beam-former are simply too great when compared to increasing the length of the equalizer. Hence, the length of the beam-former should be limited to around 2 symbol lengths and the length of the equalizer should be roughly the same as the delay spread of the channel.

After the optimal set of parameters has been determined, the second part of the chapter discusses some of the properties of the combined beam-former and equalizer architecture. The combined beam-former and equalizer provides several advantages over the traditional MMSE-based beam-former. First of all, Chapter 3.4.I has demonstrated that the combined beam-former and equalizer architecture has a wider acquisition range. Secondly, Chapter 3.4 .2 has shown that the combined beam-former and equalizer architecture is able to combine signals from multiple signal paths, which gives it a large delay spread. However, because the combined beam-former and equalizer shares the same error signals for the adaptive algorithms in the beam-former and equalizer, it suffers from a number of inherent problems. First of all, beam-formers become very sensitive to the magnitude of the error signals; therefore, the beam-former can easily become unstable. Secondly, the convergence time is slightly longer when compared to a perfectly tuned traditional beam-former. Finally, the forward filter in the equalizer has introduced a variable amount of delay for the error signal, which feeds back to the beam-former. Since the error signal latency has a direct impact on the convergence and BER performance of the beam-former, the error signal latency can be optimized in order to obtain better system performance. Chapter 4 will examine a number of strategies that can be used to improve the overall performance of the combined beam-former and equalizer. 


\section{Variable Feedback Delay Beam- former Equalization}

From the analysis performed in the previous section, it is clear that a combined beam-former and equalizer receiver system has a lower BER than the traditional separated beam-former and equalizer architecture. However, the combined beam-former and equalizer receiver system has a number of drawbacks: first, the overall receiver becomes very sensitive to the magnitude of the MSE signal; secondly, it convergences slowly; and thirdly, the latency of the feedback error signal for the beam-former is variable. The first two problems are inherent to the architecture of the receiver and are, therefore, quite difficult to improve. On the other hand, the variable error feedback signal latency can be addressed by changing the beam-former architecture to adopt the error signal delay, $d_{h f}$, to maximize BER. This section will explore the different architectures designed to resolve the variable feedback error delay problem.

The first architecture adjusts the latency of the desired signal going into the LMS algorithm to match the latency of the channel. The second architecture changes the latency of the error feedback signal going to the beam-former. There are two variants for this architecture type. The first design adjusts the latency of the error signal going to beam-former directly by inserting the appropriate number of delay stages to keep the resultant latency constant. The second design modulates the loop deliy constant of the LMS algorithm in the beam-former. 
In this chapter, each of the architectures mentioned above for mitigating the variable feedback latency problem will be analyzed and simulated. We will then compare their performances by examining the simulation results, based on the resulting BER and computational complexity.

\subsection{Systems to Equalize the Variable Error Feedback Delay}

Several solutions have been attempted to resolve the variable error feedback delay problem. In all cases, additional delays are inserted, so that the total error signal loop delay remains constant. The following section describes the different solutions attempted: modulation of the desired signal delay feeding into both the equalizer and the beam-former; modulation of the desired signal delay feeding into the LMS adaptive beam-former; modulation of the error signal delay feeding into the LMS adaptive beamformer; and finally, modulation of the loop delay parameter of the LMS adaptive beamformer. The properties for each solution will be discussed. All of the simulations run in this section are based on the channel and system parameters described in Table 5 and Table 6.

Table 5 The following table shows the value for each of the channel parameters.

\begin{tabular}{|l|l|l|}
\hline $\begin{array}{l}\text { Parameter } \\
\text { Name }\end{array}$ & Value & Description \\
\hline$K_{m}$ & 3 & $\begin{array}{l}\text { The number of message signal paths is set at three, to } \\
\text { limit computation time. }\end{array}$ \\
\hline$K_{i}$ & 2 & $\begin{array}{l}\text { The number of interference signal paths is set at two, to } \\
\text { limit computation time. }\end{array}$ \\
\hline
\end{tabular}




\begin{tabular}{|l|l|l|}
\hline $\begin{array}{l}\text { Parameter } \\
\text { Name }\end{array}$ & Value & Description \\
\hline $2 W N_{0}$ & $-15 \mathrm{~dB}$ & $\begin{array}{l}\text { The power of the Gaussian noise is limited to maintain } \\
\text { an SNR (transmitter power over receiver noise power) } \\
\text { of } 15 \mathrm{~dB} \text {. When interference is added, the noise power } \\
\text { remains constant while the SINR drops. }\end{array}$ \\
\hline$\left|\tau^{\prime \prime}-\tau^{\prime}\right|$ & N/A & $\begin{array}{l}\text { The delay spread of the channel delay varies from } \\
\text { simulation to simulation; however, it is always kept at a } \\
\text { maximum of } 6 \text { symbol times. The lower and the upper } \\
\text { bounds are arbitrarily set to meet the delay spread } \\
\text { requirement. }\end{array}$ \\
\hline$\alpha_{k}$ & N/A & $\begin{array}{l}\text { The amplitude of each signal path is randomly chosen } \\
\text { with } E\left|\alpha_{k}\right|=1 .\end{array}$ \\
\hline$\theta_{k}$ & N/A & $\begin{array}{l}\text { The angle of arrival for each signal path is arbitrarily } \\
\text { chosen during each simulation since the angle of arrival } \\
\text { doesn't significantly affect the performance of the } \\
\text { system. }\end{array}$ \\
\hline
\end{tabular}

Table 6 The following table shows the value for each of the transmission system parameters.

\begin{tabular}{|c|c|c|}
\hline $\begin{array}{l}\text { Parameter } \\
\text { Name }\end{array}$ & Value & Description \\
\hline$N_{A}$ & 3 & $\begin{array}{l}\text { The number of antennas is limited to three because the } \\
\text { smart antenna system is able to generate } N_{A}-1 \text { nulls to } \\
\text { cancel the two sources of interference. (Interference } \\
\text { comes from two different signal paths.) }\end{array}$ \\
\hline$\eta$ & 8 & $\begin{array}{l}\text { The number of samples per symbol is set to } 8 \text { to limit } \\
\text { the required length of the beam-former and the } \\
\text { equalizer. }\end{array}$ \\
\hline$\chi$ & 4 & $\begin{array}{l}\text { The down-sampling factor, or the fractional step size, } \\
\text { of the matched filter is set to } 4 \text {. }\end{array}$ \\
\hline$\beta$ & 2 & $\begin{array}{l}\text { The down-sampling rate of the feedforward filter is set } \\
\text { to } 2 \text {. }\end{array}$ \\
\hline$T$ & I us & The symbol rate of the system is set to I Msymbols/s. \\
\hline$\xi$ & 1 & $\begin{array}{l}\text { The update rate of the beam-former LMS algorithm is } \\
\text { set to } 1 \text { to improve the convergence characteristics of } \\
\text { the system. }\end{array}$ \\
\hline$\rho$ & 0.5 & $\begin{array}{l}\text { The distance between the antennas is set to half the } \\
\text { wavelength of the carrier frequency. }\end{array}$ \\
\hline
\end{tabular}




\begin{tabular}{|l|l|l|}
\hline $\begin{array}{l}\text { Parameter } \\
\text { Name }\end{array}$ & Value & Description \\
\hline$f_{r}$ & $2.1875 \mathrm{GHz}$ & $\begin{array}{l}\text { The carrier frequency of the system is set to 2.1875 } \\
\text { GHz to mimic the carrier frequency used in the } \\
\text { experimental capture system described in section 5. }\end{array}$ \\
\hline$\tau_{0}$ & 0 & $\begin{array}{l}\text { Since the transmission system is simulated in baseband, } \\
\tau_{0} \text { becomes irrelevant to the system because there is no } \\
\text { frequency lock requirement. }\end{array}$ \\
\hline$\alpha$ & 1 & $\begin{array}{l}\text { Because bandwidth is not an issue in the simulated } \\
\text { environment, the roll off factor of the pulse shape filter, } \\
\alpha, \text { is set 1 to improve the SNR. }\end{array}$ \\
\hline$L_{B r}$ & 12 & $\begin{array}{l}\text { The length of the beam-former is set to 1.5 symbol } \\
\text { lengths, which is 12, to limit simulation time. }\end{array}$ \\
\hline$L_{r r}$ & 32 & $\begin{array}{l}\text { The length of the feedforward filter is set to 32 to allow } \\
\text { a larger delay spread in the channel. }\end{array}$ \\
\hline$L_{r B}$ & 16 & $\begin{array}{l}\text { The length of the feedback filter is set to 16 to allow a } \\
\text { larger delay spread in the channel. }\end{array}$ \\
\hline$N_{t}$ & N/A & $\begin{array}{l}\text { All of the simulation results presented in this section } \\
\text { are based only on the training sequence. }\end{array}$ \\
\hline
\end{tabular}

\subsection{Modulate the Delay of the Desired Signal}

One approach to removing the variance of the error signal delay is to adjust the latency of the desired signal going to the equalizer as well as the beam-former. In Figure 22 , the delay processor uses the coefficients of the feedforward filter to determine the proper delay of the desired signal path, such that the total error signal delay remains constant. The delay processor picks the equalizer coefficient with the highest magnitude and uses the location of the tap value as an input to the delay calculation. (A linear relationship is used because the latency of the output signal is linearly related to the location of the tap value.) The design signal delay value, $d_{d s}$, is given by 


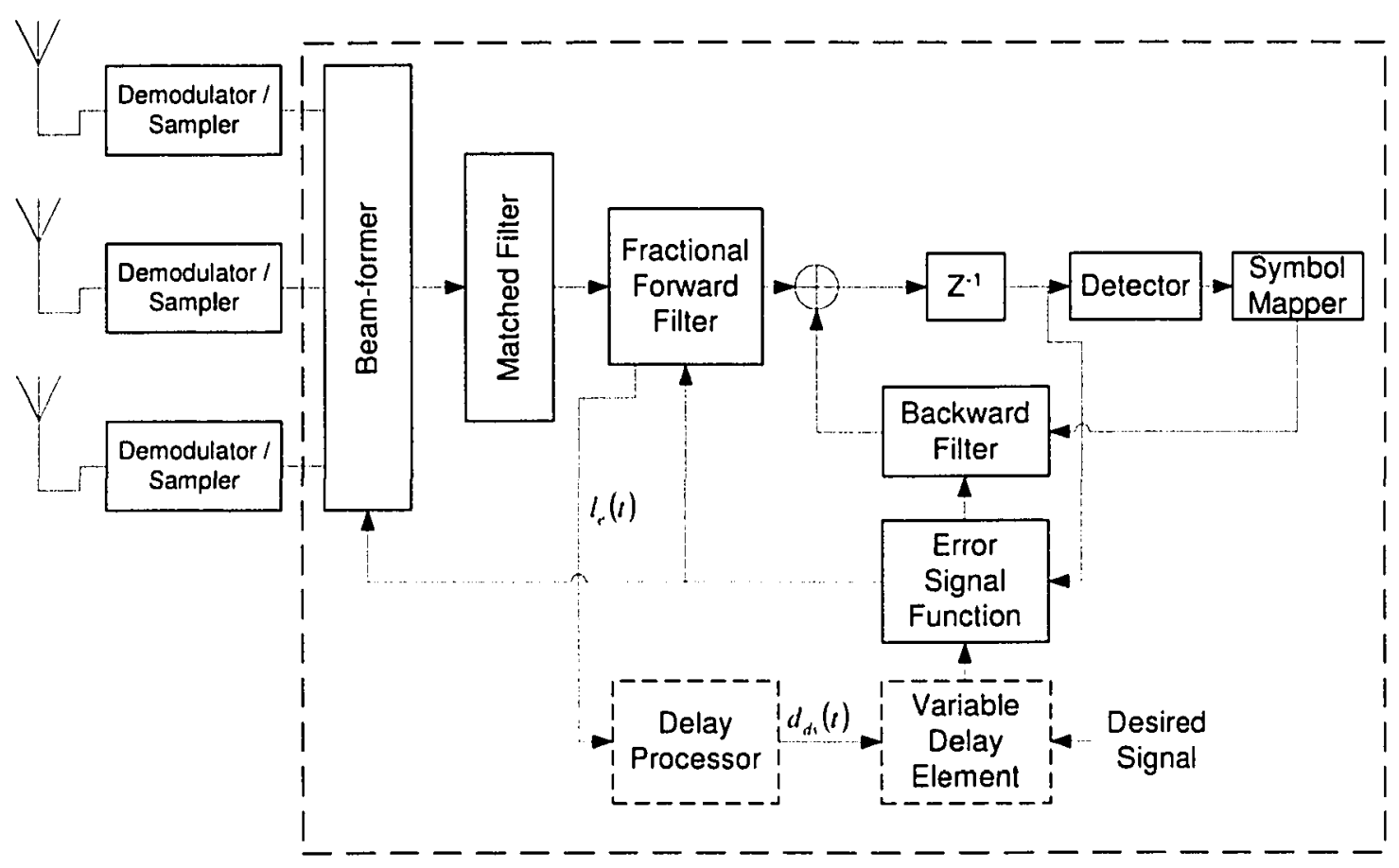

Figure 22. The desired signal is delayed such that it shifts the peak of the lap values to the center; hence, the error signal loop delay becomes constant. The delay processor measures the delay introduced by the feedforward filter and adjusts the variable delay element to compensate.

$$
d_{d s}(t)=\int\left(l_{e}(t)-c_{e}\right) \times c_{d s} d t+d_{c}
$$

where $l_{\text {e }}$ is the coefficient number of the tap with the highest value in the feedforward filter, $c_{c}$ is the location of the center tap of the feedforward filter, $c_{d}$ is the rate for the $d_{d,}(t)$ in coverage to a known value, and $d_{i}$, is the inherent channel delay plus receiver component delay. According to this equation, the FSE coefficient with the highest value would eventually be moved to the center of the equalizer. As the delay of the desired signal varies, the magnitude of the coefficients will also change, and it takes the system some time to reach a steady state. Hence, the desire signal delay $d_{d}$ can only be updated 
every $s_{\text {update }}$ samples, where $s_{\text {update }}$ should be set to a large value to prevent the system from going into an unstable state. As a result, the loop delay of the error signal going to the adaptive beam-former will stabilize eventually.

However, there are several problems with this scheme. First of all, as the desired signal input into the equalizer changes, it perturbs the equilibrium in the equalizer. Because of the slow convergence time of the system, this can be a problem if the delay of the channel changes over a short period of time. Furthermore, the equalizer and the beam-former can go out of lock altogether and it will take a long time for the receiver to re-acquire the signal again if the SNR is high. Moreover, because the system can only make updates every $s_{\text {updute }}$ samples, it will $t^{\prime}: e$ the system much longer to converge. Secondly, in the case of a very large latency spread between different signal paths, the two strongest signals might be near the two ends of the equalizer. By shifting the desired signal, one of the strongest signals will move to the center of the equalizer and push the other signal out of the acquisition range of the system. Therefore, the system will not be operating at the most favorable point, which will reduce the overall performance of the system. In addition, if the signal path for the strongest signals has changed, the system might not be able to detect the other signal again; hence, the system will be stuck in a deadlocked state. Figure 23 shows the BER performance of both an uncompensated combined beam-former and equalizer and a desired signal modulated beam-former and equalizer system. The BER is averaged over 9 different simulations with a random set of channel parameters, limited by the constraints in Table 5 . The simulation has demonstrated that the BER performance of the compensated system is slightly better than 


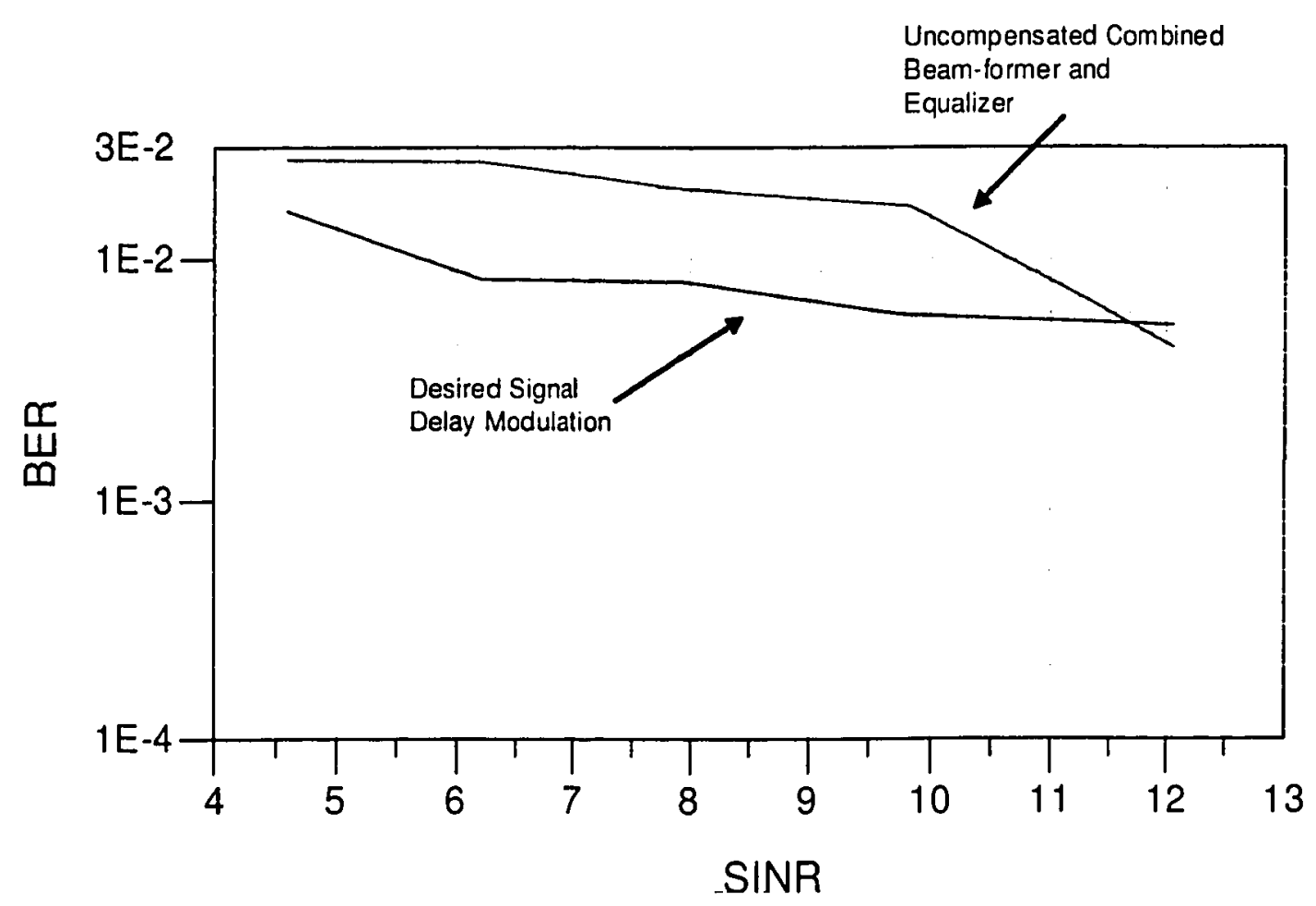

Figure 23. The graph compares the BER of the combined beam-former and equalizer with the desired signal delay being modulated against the traditional beam-former design.

the uncompensated combined beam-former. This is probably due to the instability of the desired signal latency and the time it lakes for the system to converge.

\subsection{Modulate the Delay of the Desired Signal Delay to the Beam-former}

To address the convergence problem observed in the previous design, one can separate the desired signal going to the beam-former and the equalizer, and adjust only the latency of the desired signal going to the beam-former. Hence, changing the latency of one will not significantly perturb the other. There are two implementations of the delay processor that can be used to achieve the above design goal. In the first design, the delay processor uses the tap values in the equalizer as the input. In the second design, a 
separate equalizer is built, and the delay processor uses the tap values from the second equalizer as inputs.

\subsubsection{Desired Signal Delay Model I}

In the original architecture, the error function is based on the output of the beamformer and the desired signal. The resulting error signal is fed directly to the beamformer to update the weights of the beam-former; hence, this design doesn't have the problem of variable error signal delay. However, because the number of taps used in the beam-former is usually smaller than that of the equalizer, the acquisition range is much smaller. To address this problem, the desired signal going to the beam-former, as shown in Figure 24 , is delayed by a programmable delay element to match the latency of the incoming signal. The variable delay element is controlled by the delay processor in the same manner as the other design.

One of the advantages of this architecture is the fast convergence time of the beam-former because the error signal feedback loop is much smaller. However, there is a fundamental problem with this design. When the desired signal fails to match the delay of the incoming signal, the beam-former will approach a zero solution. This is a property of the LMS algorithm. When the desired signal and the incoming signal are completely uncorrelated, the LMS algorithm treats the incoming signal as noise. Hence, a zero solution will be produced, forcing the output of the beam-former to become zero. This problem causes the system to be in a deadlocked situation, because when the signal, which the beam-former has locked onto, suddenly changes its latency, the reaction time of the equalizer is not fast enough to change the delay of the desired signal for the beamformer to compensate. Hence, there will be no available signal within the acquisition 


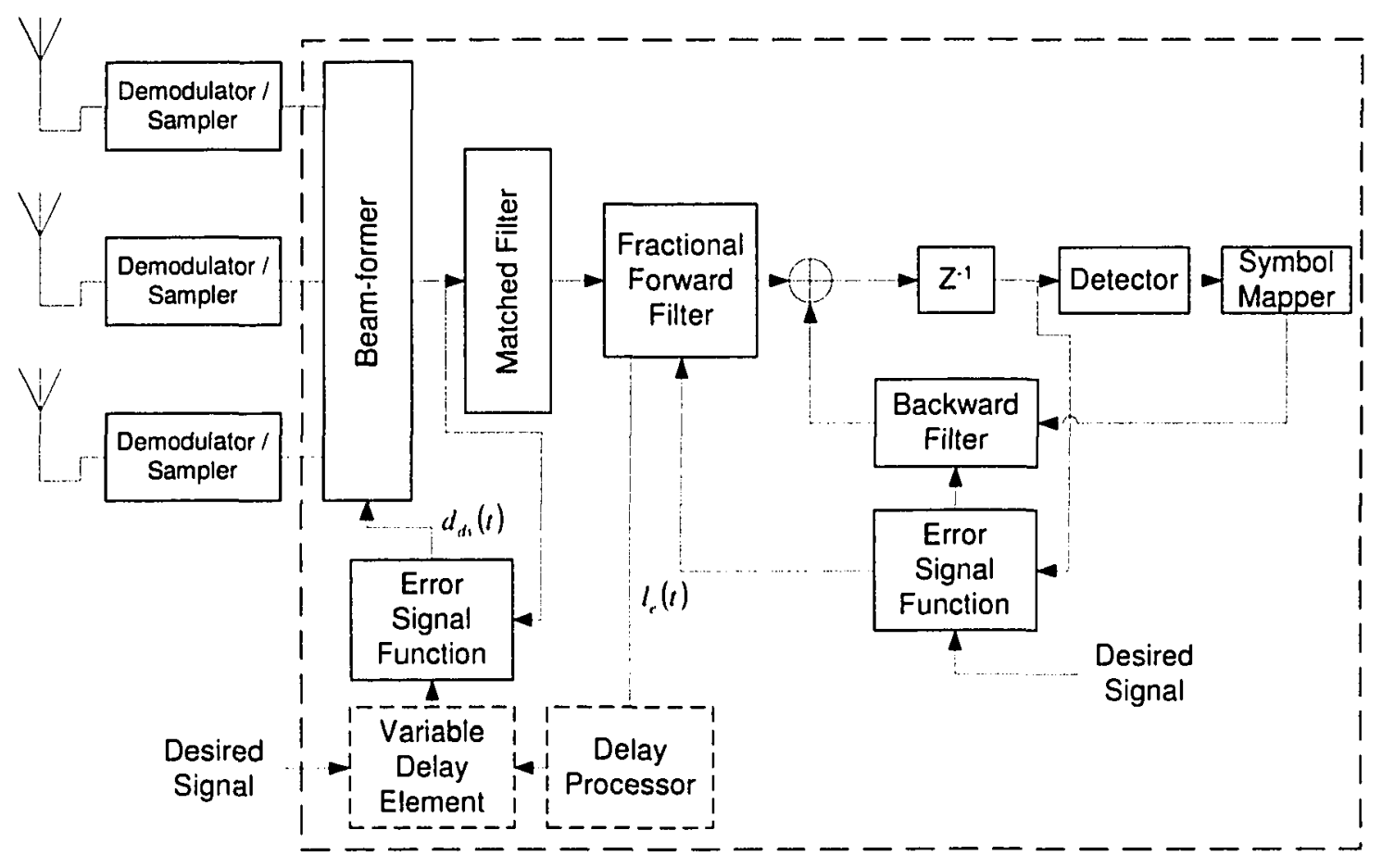

Figure 24. The delay processor calculates the proper delay value and programs the variable delay element such that the desired signal matches up with the incoming signal to the beam-former.

range of the beam-former and the LMS algorithm will drive the weight of the beamformer to produce zero output. When the zero output is fed into the equalizer, the inputs become completely uncorrelated with the desired signal and the equalizer is driven to a zero solution as well. Since the zero solution is a stable solution, the weight will not be updated. Consequently, the system will fail to function until another signal within the acquisition range of the beam-former appears. In addition, when the system using the desired signal delay model $\mathrm{I}$ is being simulated, the system turns out to be extremely unstable. Both the beam-former and the equalizer fail to converge to a stable solution.

\subsubsection{Desired Signal Delay Model II}




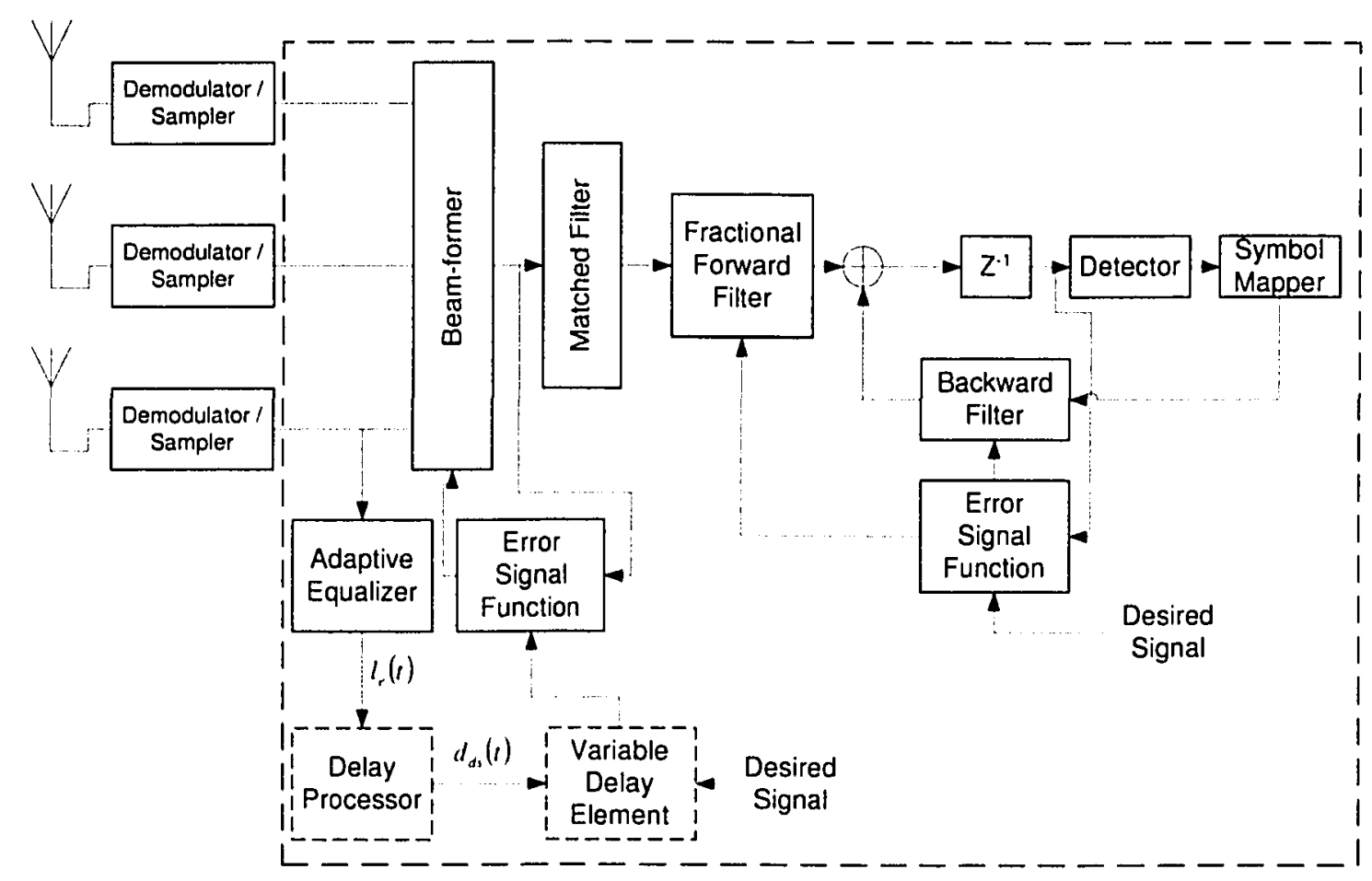

Figure 25. The secondary equalizer uses signals from one of the antennas as input. Although the signal is not enhanced by the beamformer, the equalizer is still able to pick up the desired signal in the face of severe interference.

In order to correct the problems stated above, a second design has been tested, as shown in Figure 25. Instead of using the delay signal from the equalizer after the beamformer to determine the delay of the desired signal feeding into the beam-former, a second equalizer is used to determine the appropriate delay for the desired signal. The second equalizer is fed directly by one of the antenna's outputs. Since the inputs of the secondary equalizer are not processed by the LMS adaptive beam-former, the secondary equalizer will be able to continue to monitor the incoming signal even though the beamformer approaches the zero solution. As the signal within the beam-former acquisition 
range disappears, the secondary equalizer is able lock onto another signal with its acquisition range; thus, the beam-former is able lock onto the new-found signal.

Although this design resolves some of the flaws presented in the previous design, this new design itself has other problems. First of all, this design still uses only one copy of the transmitted signal instead of all the available delay versions of the transmitted signals; as a result, the performance of the system is reduced. Secondly, the input signal is not enhanced by the beam-former; therefore, the coefficients of the equalizer can fluctuate due to noise and other interferences. As a result, the latency value of the desired signal can change quite rapidly, which perturbs the beam-former; consequently, the BER performance of the system might be affected.

Figure 26 shows the BER performance averaged over 9 different random simulations. Similar to the desired signal modulation scheme, the beam-former desired signal modulation design offers slight BER improvements. This is probably as a result of the problems mentioned above. 


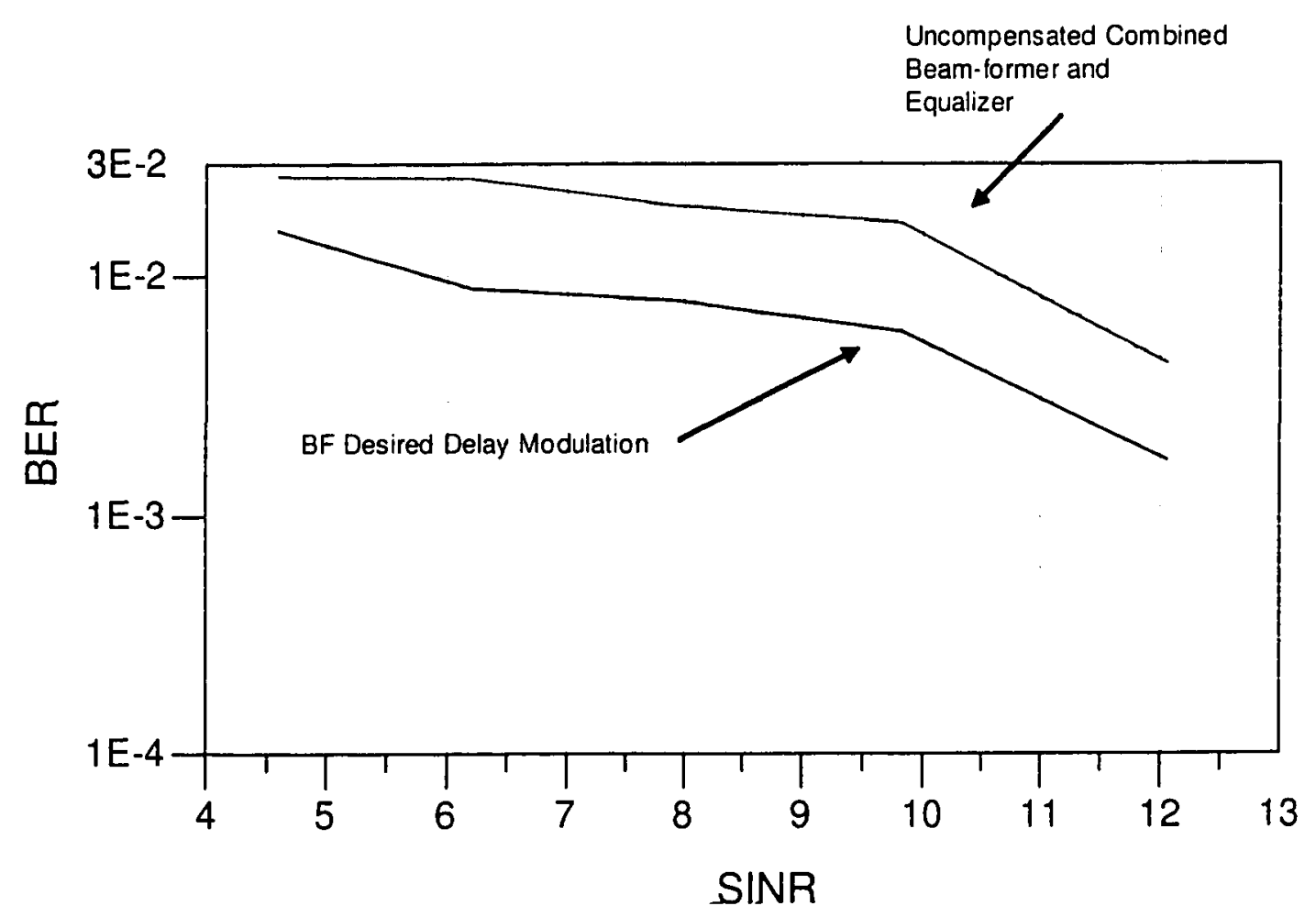

Figure 26. The graph compares the BER of the combined beam-former and equalizer with the desired signal delay being modulated against the traditional beam-former design.

\subsection{Modulate the Delay of the Error Signal to the Beam-former}

Instead of adjusting the delay of the desired signal, another approach is to adjust the latency of the error signal feeding back to the beam-former. The error signal going to the beam-former in Figure 27 is delayed by a variable delay element, and the delay value is driven by the delay processor, which takes its input from the tap value of the feedforward filter.

The design of the delay processor is an integrator, given by: 


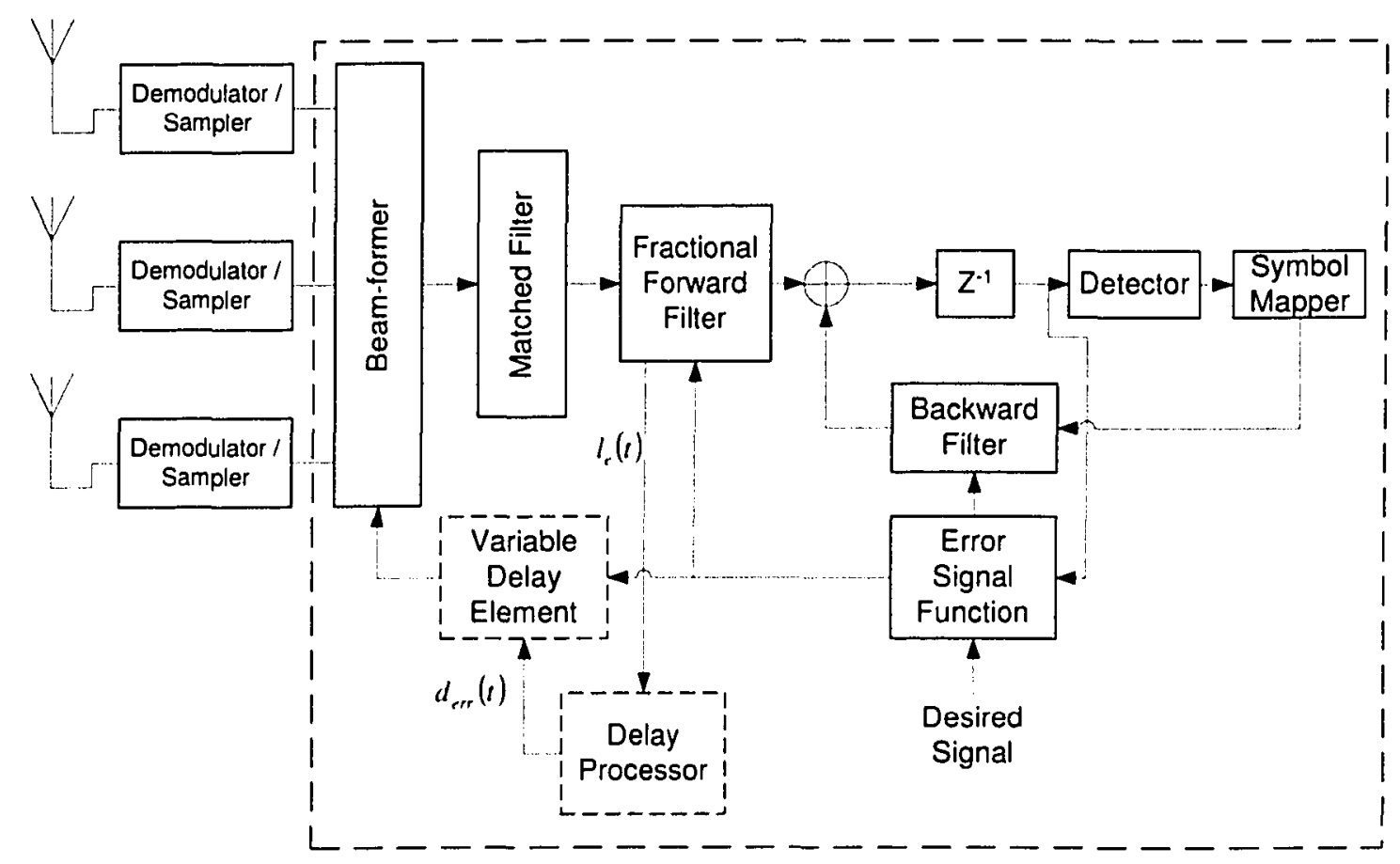

Figure 27. By modulating the delay of the error feedback, the total loop delay could stay constant because the variable delay unit could compensate for any additional delay introduced by the FSE

$$
\begin{aligned}
& d_{b f}(t)=d_{l o o p}-\left(d_{c^{\prime} r r}(t)+d_{c b f}\right) \\
& \frac{\mathrm{d} d_{e r r}(t)}{\mathrm{d} t}=\left(\left(l(t)-c_{e^{\prime}}\right)-d_{e r r}(t)\right) \times c_{e^{\prime} r r}
\end{aligned}
$$

where $d_{t r r}(t)$ is the output of the delay processor, which is fed into the variable delay element to add latency to the error signal, $c_{c r}$ is the rate at which the system should converge to the steady state solution, $d_{c \cdot b j}$ is the delay of the error signal when the highest value in the equalizer is the center tap, and $d_{\text {lomp }}$ is the maximum delay that can be added by the equalizer plus the delay of all the intermediate components. Using the variables defined in (52), (39) can be re-written as 


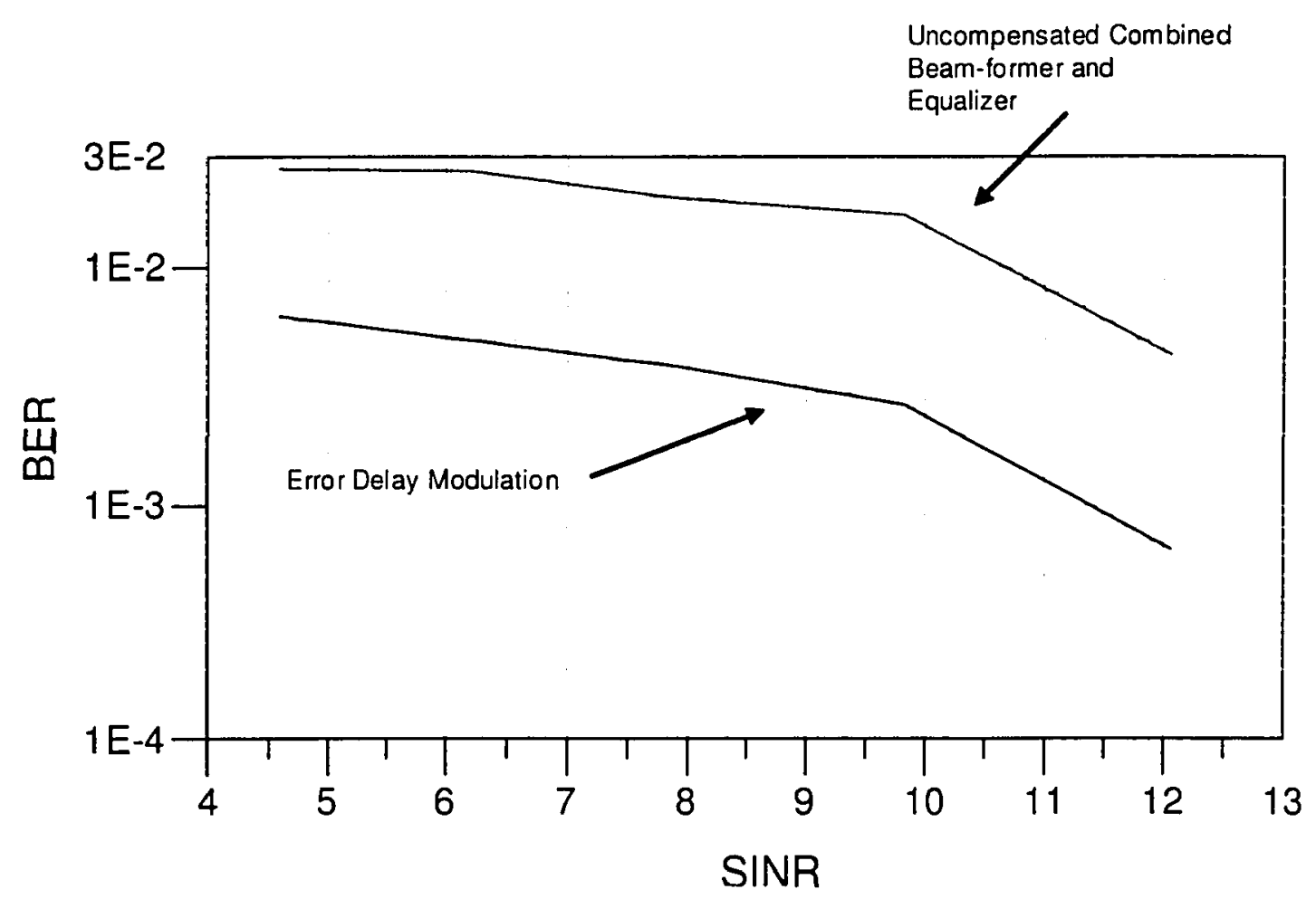

Figure 28. The graphs on the right are the BERs of the system that uses the error signal delay modulator, and the graphs on the left are the BERs of the original combined beam-former and equalizer system.

$$
\rho_{a . l}(m+1)=\rho_{a . l}(m)-2 \mu r_{a . \eta\left(m-d_{l m m}\right)-l}\left(z_{m-d_{r m}}-v_{m-d_{r r r}}\right)^{*} .
$$

Figure 28 clearly shows that delay modulation systems can significantly improve the BER of the system. Moreover, if one were to observe the changes in the BER over time, one would be able to conclude that the delay modulation system helps the receiver to converge to a stable solution faster than the original system. This can be confirmed by looking at the magnitude of the MSE signals for the two systems shown in Figure 29. 
Beam-former with Error

Signal Delay Modulation
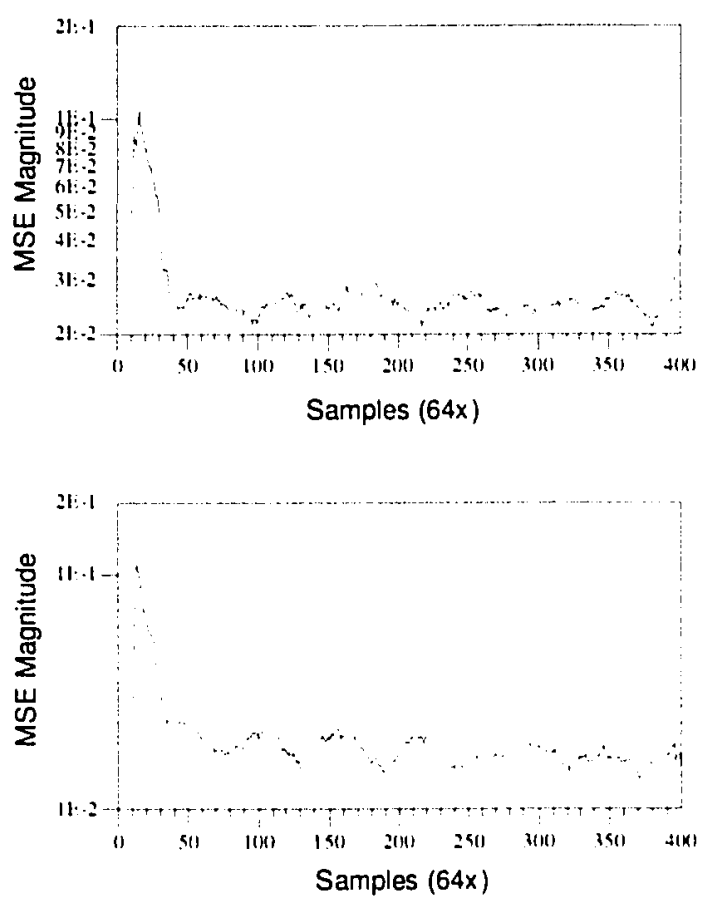

Original Combined Equalizer and Beamformer
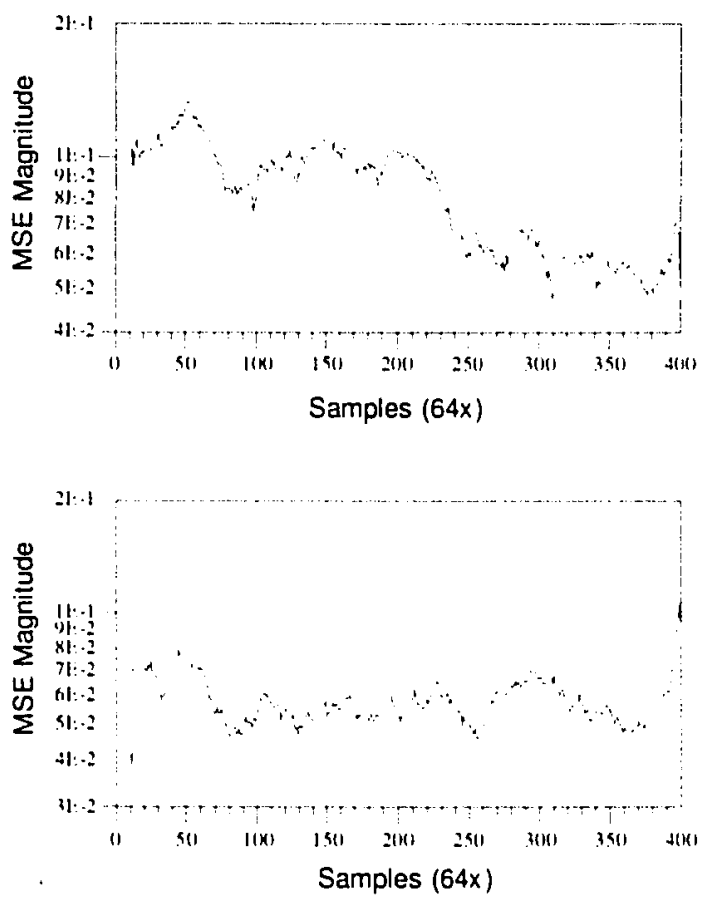

Figure 29. The graph shows the magnitude of the MSE signal for two random simulations.

In addition, this particular design has several advantages over all of the previously discussed architectures. One advantage of this design is that it does not require any changes to the design of the equalizer and the beam-former because only the latency of the error signal is being changed. Secondly, the system should be more stable because, even in the absence of a delay processor, given enough time and signal strength, the system will eventually converge to a solution: hence. by adjusting the error signal delays only, the system should not easily become unstable. Thirdly, since the system only adjusts the error signal going to the beam-former during the acquisition phase, the tap 
value of the equalizer should not change dramatically; hence, the stability of the system is further enhanced. Finally, this design doesn't limit the acquisition range of the receiver. If one of the paths of the transmitted signal is blocked, the receiver will be able to switch to another path.

\subsection{Modulate the Loop Delay Constant in the Beam-former}

With some slight modifications to the LMS algorithm for the beam-former, one can easily adapt the delay processor to adjust $d_{h j}$ and (39) can be written as

$$
\rho_{a, l}(m+1)=\rho_{a, l}(m)-2 \mu r_{a, \eta\left(m-\left(d_{l m, m}-d_{e m}\right)\right)-1}\left(z_{m}-v_{m}\right)^{*}
$$

Although the changes look trivial, the memory requirement for adjusting $d_{l f}$ directly is half of that required for modulating the delay of the error signal going to the beamformer. This is because modulating the delay of the error signal requires extra memory to store the delayed version of the error signal; on the other hand, modulating the loop delay constant does not need a delayed version of the error signal.

Based on the simulation data shown in Figure 30, modulation of the loop delay constant improves the overall performance of the receiver system, when compared against the original beam-former architecture. In addition, Figure 31 has shown that the BER performance of the error loop delay modulation is superior to the error delay modulation. Consequently, modulation of the loop delay constant seems to be the best method for mitigating the variable error signal delay problem because of the comparable performance and the reduced memory requirement. 


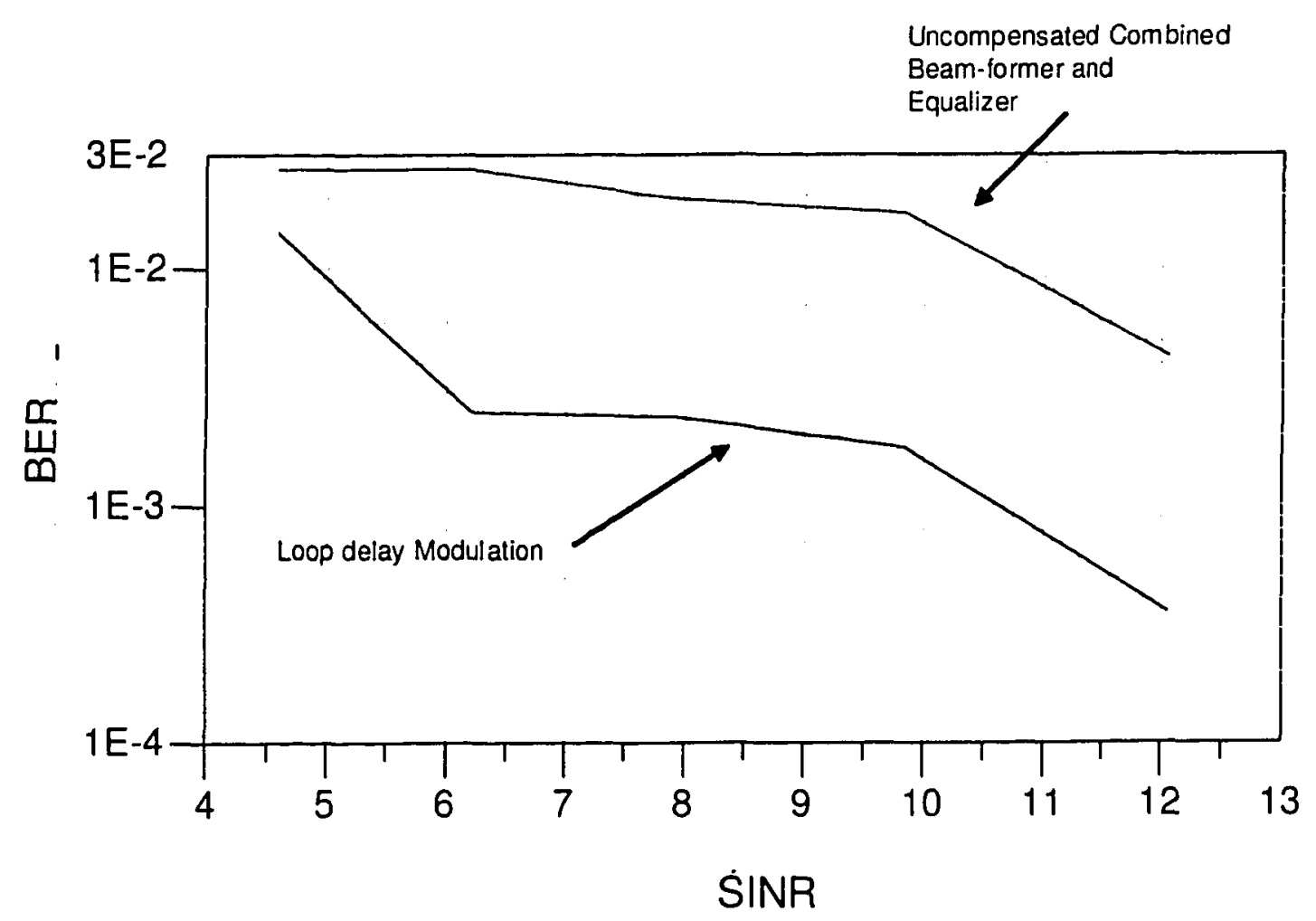

Figure 30. The graph shows the BER of the loop delay constant modulation system against an ordinary combined beam-former and equalizer system. 


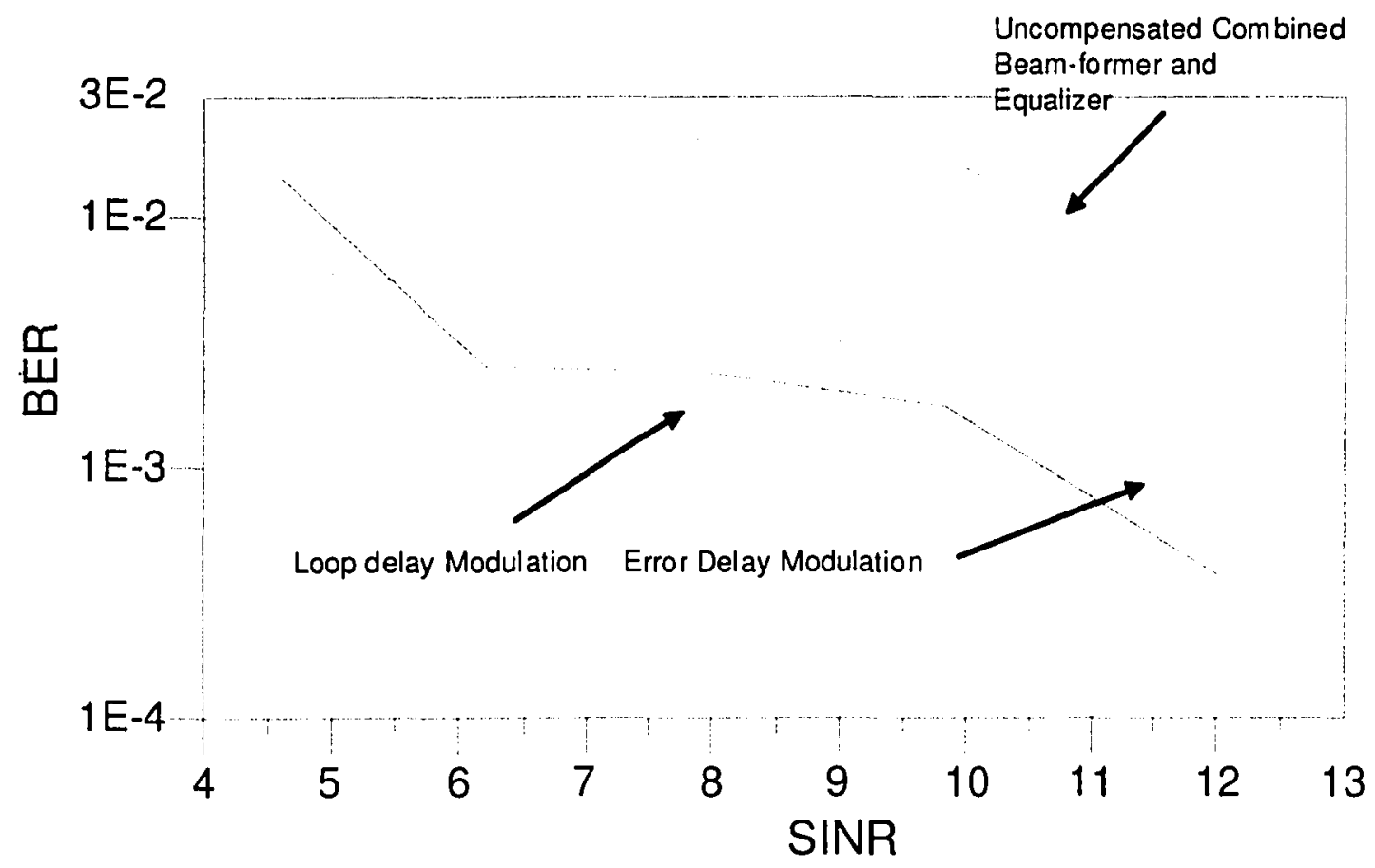

Figure 31. The graph shows the BER of the loop delay constant modulation system against the system, which modulates the delay of the error signal to the beam-former.

\subsection{Design Comparison}

By examining the results obtained from the simulations, one can easily conclude that adjusting the delay of the desired signal makes it a lot harder for the system to converge. Although both types of designs are able to slightly enhance the overall BER performance of the receiver, the benefits are limited and incur additional hardware resources. On the other hand. the designs that focus on adjusting the delay of the error signal feeding back to the beam-former produce far better BER performance gains and lower the additional causes of system complexity. . Furthermore, by adjusting the loop delay parameter in the update equation directly, not only is the hardware overhead lowered but also the performance gain is slightly better than in the design that attempts to 
delay the error signals feeding back to the beam-former. Consequently, loop delay parameter modulation is the best of the four different designs discussed for solving the variable feedback error delay problem. 


\section{Signal Capture System}

In order to show the performance gain as a result of the improvements made to the traditional DAB design, a $2.1785 \mathrm{GHz}$ signal capture system has been built to record real-life data in order to test the proposed modifications [12][13][14]. As with many wireless systems, in order for the capture system to work successfully, several hardware and system level parameters need to be considered $[16)$ :

1. The carrier, symbol, and frame synchronization between the transmitter and the receiver.

2. The format of the transmitted signal.

3. The design of the adaptive algorithms.

This chapter will examine each of the above concerns and present some of the solutions to mitigate these problems. The first section contains a brief description for each of the hardware components in the capture system. In the second section, the transmitted signal format is discussed. In the third section, two different adaptive algorithms are presented, along with an analysis of how each of the algorithms can improve the convergence of the adaptive system. In the final section, we will examine the environments in which the capture system is set up to collect data for analysis.

\subsection{Capture System}

The capture system is built to record the base-band data signal, which is then streamed to a file in a PC for off-line processing. Figure 32 shows the four major 


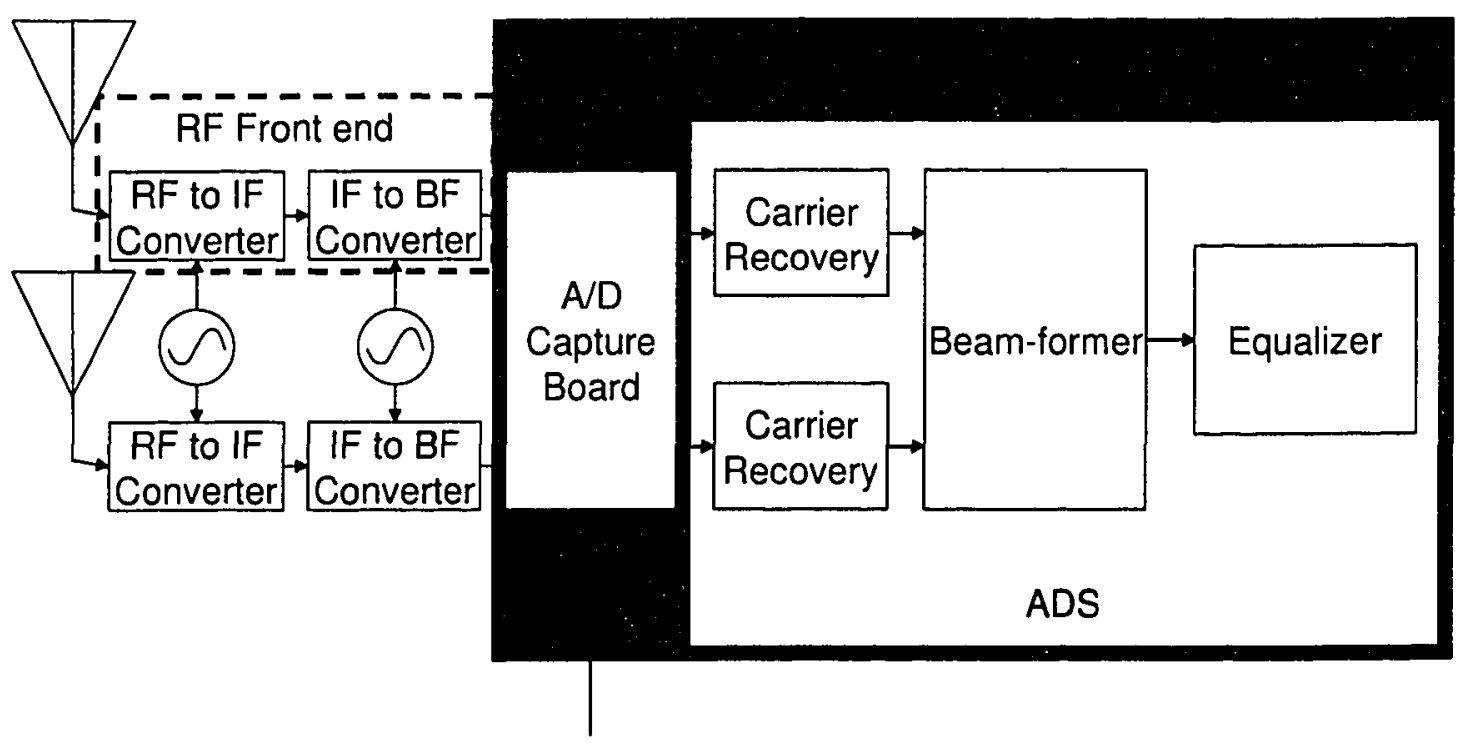

$2 \times$ Symbol Clock from transmitter

Figure 32. The capture system consists of four major components: the antenna, the RF front-end assemblies, the A/D capture board, and the carrier recovery systems.

components of the capture system: the antennas, the RF front-end assemblies, the A/D capture board, and the carrier recovery systems. The following section provides a brief description for each of the components and the roles they play.

\subsubsection{Antennas}

The antennas used in the capture system are simple patch antennas, which only radiate in a forward direction. Figure 33 shows the shape of the antennas and their radiation patterns. Both the transmitter and the receiver use the same type of patch antenna. Therefore, all the experiments conducted are limited to signals that arrive facing the receiver. On the receiver side, two antennas are placed side-by-side to form a linear array configuration. The distance between the two antennas is approximately half a wavelength 

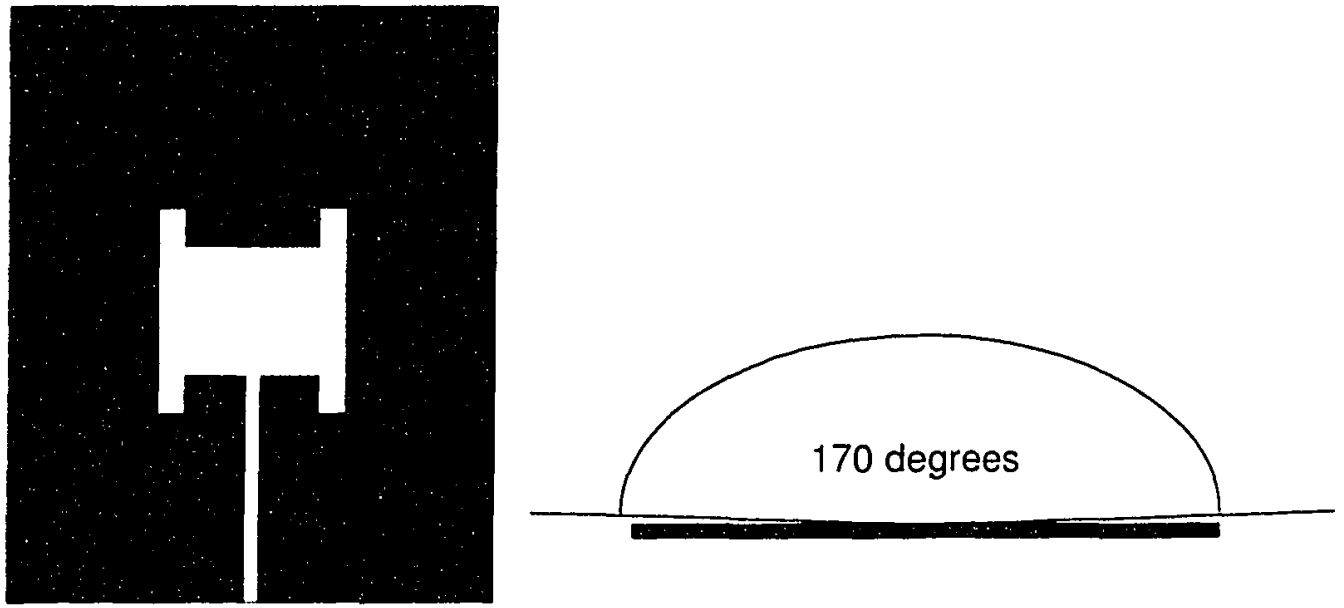

Figure 33. The antennas used in the capture system are patch antennas, which radiates only in the forward direction with beam width of 170 degrees.

\subsubsection{RF Front-end Assemblies}

The RF front-end assemblies are used to down convert the signals from the antennas before they are recorded by the A/D capture board. The RF front-end assembly is composed of a Radio Frequency to Intermediate Frequency (RF-to-IF) converter, and an Intermediate Frequency to Base-band Frequency (IF-to-BF) converter (both of which are pre-assembled modules obtained from Maxim ${ }^{T M}$ Integrated Products.) The RF-to-IF converter requires a $1 \mathrm{GHz}$ reference clock and the IF-to-BF converter requires a $10 \mathrm{MHz}$ reference clock; the reference clocks are shared between the two front-end assemblies. Because the converters come pre-assembled, the operating frequency is limited by the components used in the modules: therefore, the RF front-end assembly is capable of handling frequencies of only up to $2.1875 \mathrm{GHz}$. (We feel that $2.1875 \mathrm{GHz}$ is close enough to many of the current wireless transmission standards: therefore, system should be representative of a real life wireless transmission system.) The outputs of the RF 
front-end assemblies are a pair of in-phase and quadrature-phase signals, which are connected to the A/D capture board.

\subsubsection{A/D Capture Board}

The A/D capture board records the signals received by the RF front-end assemblies, which are streamed to the hard disk of a PC. Because the system bus in the PC is limited to $33 \mathrm{MHz}$, the capture board is only capable of streaming data to the hard disk at a maximum rate of $250 \mathrm{Ksamples} / \mathrm{s}$. Therefore, the maximum symbol rate is limited to $125 \mathrm{Ksymbols/s.} \mathrm{Afterwards,} \mathrm{the} \mathrm{beam-former} \mathrm{and} \mathrm{equalizer} \mathrm{implemented} \mathrm{in}$ $\mathrm{ADS}^{\mathrm{TM}}$ read the recorded data from the signal sample file and process them. (Although an on-line processing system is desired, it is believed an off-line processing system should be sufficient to demonstrate the performance gain as a result of the improvements made to the beam-former architecture.) The symbol clock in the transmitter is connected directly to the A/D capture board, and the A/D capture board uses a multiple of the symbol clock as the sampling clock. Hence, the system will not need to perform any additional symbol clock recovery.

\subsubsection{Carrier Recovery System}

Because the transmitter and the RF front-end assemblies use two different reference clocks, even though both are tuned to the exact same frequency, there is still a slight frequency difference due to component mismatches and thermal variances. Therefore, a carrier recovery system is implemented in the $\mathrm{ADS}^{\mathrm{TM}}$ to remove the residue carrier in the captured signal before the samples are processed by the beam-former and 
the equalizer. Since the transmitted data is modulated using 4-QAM, a simple Costasloop is used to remove the carrier from the received signal. [17]

In Figure 32, the carrier recovery system is placed immediately after the A/D capture board. Ideally, the carrier recover system should be placed after the beam-former to take the benefit of the higher signal-to-noise ratio (SNR). However, because of the component mismatches in the RF front-end assemblies, the frequency of the residue carrier from each front-end assembly is slightly different. Hence, it is impossible to use the beam-former to combine the signal from the two antennas first before the carrier is removed. (Refer to Appendix A for a detailed discussion of the Costas-loop and components mismatch problem.)

\subsection{Pilot Signal Modulation}

The LMS algorithm implemented in the receiver requires some known data bit, or training symbols, in order for the algorithms to converge. There are two requirements for the design of the training signal:

1. The training signal needs to be sent periodically in order for the receiver to update the weights in the beam-former and in the equalizer as the environment changes. This requirement introduces another layer of synchronization between the receiver and the transmitter. Given that there is only one communication channel between the receiver and the transmitter, it can be very difficult to implement.

2. The training signal should travel the same path as the data signal in the same channel environment. This should be an easy requirement to fulfill as long as the training signal is sent frequently enough. 


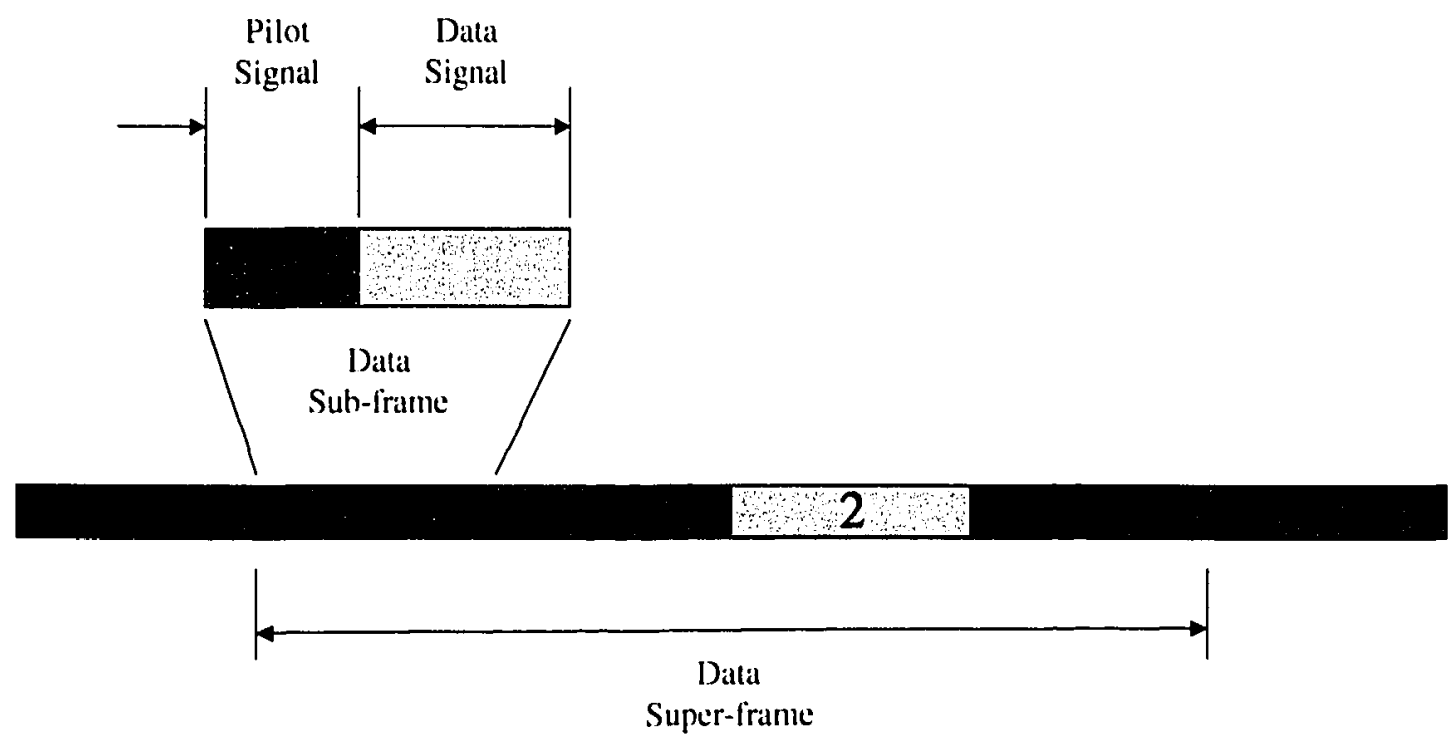

Figure 34. The pilot signal is embedded in the data signal to help the beam-former in the receiver to home in on the signal. The ratio of the pilot signal to the data signal will determine the speed of the algorithm convergence on the receiver side.

Both of these requirements can easily be satisfied if the transmitted signal is designed carefully. One approach is to use pilot signal modulation, which embeds the training data directly into the data signal |18|. Figure 34 shows the data format used in the capture system. The data signal is segmented into different sub-frames. A data subframe consists of both pilot signal symbols and data symbols; the system is designed to have a data to pilot signal ratio of $2: 1$. Four data sub-frames together form a data superframe. The pilot signal sequence changes in each sub-frame and it repeats every superframe. Given that the bit rate is around $250 \mathrm{Kbit} / \mathrm{s}$, or $125 \mathrm{Ksymbol} / \mathrm{s}$, it takes $384 \mathrm{us}$, or 48 symbols, for the pilot signal sequence to repeat once. The system actually functions better if the pilot signal is random, or at least pseudo-random. However, if the pilot signal were never to repeat, the transmitter and receiver would be required to synchronize the pilot signal sequence. Although there are algorithms that allow the synchronization 
of pseudo-random number generators across a noise channel, this is beyond the scope of this thesis, and is not implemented. Therefore, the pilot repeats within a short period of time in order to avoid having to introduce additional synchronization constraints.

Because the pilot signal sequence repeats every 48 symbols, it imposes additional constraints on the design of the equalizer. Since the equalizer uses LMS as the adaptive algorithm, the algorithm altempts to minimize the error signal, which is the difference between the pilot signal and the received signal. Therefore, if the equalizer length were to be longer than 48 symbols, two versions of the pilot signal sequence, separated by 48 symbols, would be seen by the LMS algorithm and the algorithm would attempt to sum the two delay versions of the pilot signal from two different super-frames together: as a result, the data signal would be corrupted. (The pilot signal might be the same but the data sequences of the two super-frames would be random, and different.) In addition to the length of the equalizer, the repetition of the training sequence, every 48 symbols. poses another constraint on the environment. The latency spreads of the different signal paths cannot exceed 48 symbols; otherwise, the same problem of summing data sequences from two super-frames will occur. This is not as much of a problem as the equalizer length. In order for the latency spread of the multi-path signals not to exceed 48 symbols, the length difference of the two paths has to be at least 24 kilometers, given the system symbol rate. Since the transmitter used in the capture is relatively weak, and the signal capturing exercises are conducted in an indoor environment, it should be almost impossible for the different signal paths to have a latency spread greater than 48 symbols. 


\subsection{Adaptive Algorithms}

Since the data signal is not known beforehand, the LMS algorithm needs to be switched off during the data sequence and switched back on for the duration of the pilot signal. Although the pilot signal is able to provide some help for the adaptive algorithm to home in on the data signal, relying on the pilot signal alone is not enough for the system to completely converge. (It is shown in a simulation that only the pilot signal portion of the received signal will converge, while the data signal does not.) Therefore, two strategies have been proposed to resolve this problem. First of all, a combined adaptive algorithm, which uses both LMS and CMA, is implemented. Secondly, randomizing the symbol location of the pilot signal will help the system to converge.

\subsubsection{Combined LMS and CMA Algorithm}

The pilot signal allows the use of the LMS to update the weights of the beamformer. Since the pilot signal is known beforehand, the adaptive algorithm could simply subtract a known version of the pilot signal from the received pilot signal to generate an error signal. Using the LMS algorithm alone does not necessary produce the most optimal result because the data signal is not used as part of the calculation of the beamformer weights. Therefore, CMA is used on the data signal sequence to update beamformer weights. The CMA doesn't require that the content of the data is known beforehand. The only requirement is that the modulation scheme used produces symbols with equal amplitude. Since both adaptive algorithms generate an error signal, the two error signals are time multiplexed together before the combined signal is fed into the weight update engine. 
However, there is one major drawback with the combined LMS and CMA algorithm. Because CMA doesn't require the content of the signal, the resulting data can have an arbitrary phase [7]. On the other hand, the LMS algorithm does require prior knowledge of the signal content and the resulting signal has exactly the same phase as the pilot signal. The problem occurs between the boundary of the pilot signal and the data signal. The error signal generated by the LMS algorithm is able to retain its original phase; meanwhile, the signal produced by the CMA can have arbitrary phases. Therefore, when the receiver tries to decode the signal, an undesired transition might occur between the boundaries of the pilot and the data signal, introducing bit errors into the system.

\subsubsection{Spread Pilot Signal LMS Algorithm}

In order to resolve the boundary problem between the LMS and the CMA algorithms, the position of the pilots are different for each of the sub-frames, and the pilot sequence will only repeat for each super-frame. (A solution for the LMS is also a solution for the CMA algorithm, given that the modulation is 4QAM. However, the reverse is not always true because there is phase ambiguity in the CMA.) Figure 35 shows the positions of the pilot symbols used in the test environment.

The cause of the transition problem can be quickly understood by examining the set of equations used for the LMS algorithm. In (39), the error signal depended on the processed input and the desired signal. Because not every symbol is known in advance, this equation can only be evaluated whenever the output is the training symbol. There are two possible solutions, which the optimization process can produce. The first solution, which is the desired solution, is to optimize the value of each tap, such that the data 


\begin{tabular}{|c|c|c|c|c|c|c|c|c|c|c|c|c|}
\hline Symbols & 11 & 10 & 9 & 8 & 7 & 6 & 5 & 4 & 3 & 2 & 1 & 0 \\
\hline Sub-frame 0 & & & & & & & & & & & & \\
\hline Sub-frame1 & & & & & & & & & & & & \\
\hline Sub-frame 2 & & & & & & & & & & & & \\
\hline Sub-frame 3 & & & & & & & & & & & & \\
\hline
\end{tabular}

\section{Data Symbol $\square$ Pilot Signal Symbol}

Figure 35 . The diagram shows how the data symbols are interleaved with the pilot signal symbols. Instead of embedding the pilot signal symbols in each sub-frame, the pattern of the pilot signal symbols are repeated only during each super-frame.

output is the same as the training symbols. The second solution, which is observed during simulation, is to optimize only the taps that contain the training symbols, and let the remaining taps go to zero. If the training signal is periodic, not all the taps will have a chance to be optimized by the LMS algorithm, and the rest of taps will be optimized by the CMA algorithm; hence, there will be phase disconnect between the pilot symbols and the data symbols. On the other hand, if the pilot symbols are spread around in the superframe, the LMS algorithm will have a chance to work on all of the taps. Consequently, the LMS and the CMA algorithms can work together without generating any phase disconnect.

\subsection{Environment Setup}

In order to demonstrate the benefits of the error signal delay compensation system, the capture environment will need to have more antennas. In addition, the capture environment should also be able to emulate a large delay spread channel. However, there are several limitations to the existing capture system, which make both requirements difficult to achieve. First of all, due to limited resources for this work, there 
were only two sets of receiver elements available. It is therefore impossible to reproduce here a transmission system with a higher number of antenna elements. Secondly, the transmitter is relatively weak; therefore, the transmission range is quite short, and the capture rate of the PC limits the symbol rate of the system; hence, a single symbol time of difference requires a large difference in distances between the signal paths. As a result, a large delay spread channel is impossible to reproduce. However, both of these problems can be resolved by taking into account the relative stability of the environment and the ability to superimpose multiple capture data sets in software. The following section describes how to get around some of these problems.

\subsubsection{Multi-antenna Emulation}

In order to emulate multiple antennas, one could superimpose data sets captured from two different sessions, as shown in Figure 36, to emulate a 4-element receiver array. During the lirst test. the antennas are placed on the left hand side of the receiver array and the data is captured. Afterwards, the antennas are moved to the right hand side of the array. The same data sequence is transmitted and the data is captured with the antennas on the right hand side. Finally, both sets of captured data are compared in software in order to match their start time before the data set is fed into the beam-former.

In order for this system to work, several assumptions are made. First of all, the test environment has to remain relatively constant during the two capture sessions. During the two trials, all the objects in the environment should not move; hence, the test should be performed in-doors because it is easier to control the environment. This will guarantee that the data from the two capture sections are relatively similar. Secondly, the transmit power of the signal is relatively weak: therefore, the receiver will probably see 
Test \#1

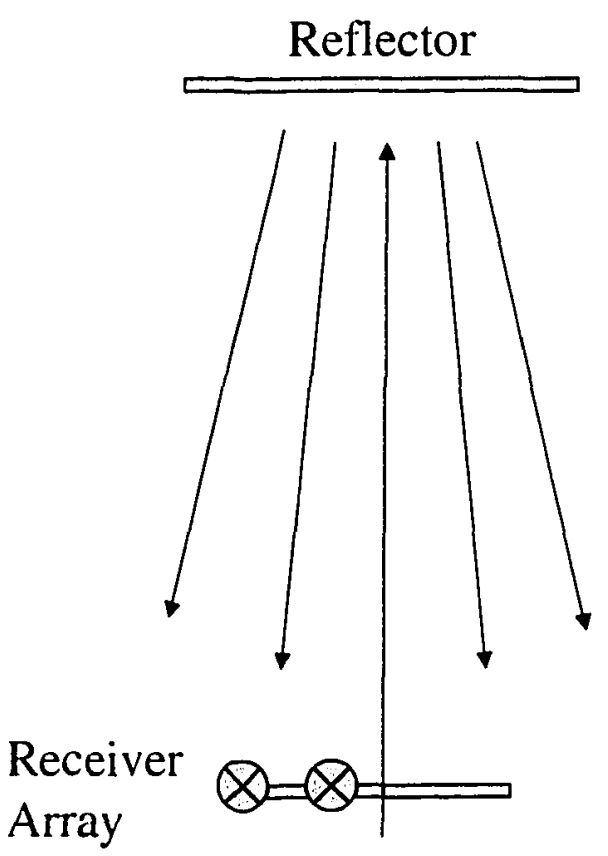

Array

$\oplus$

Transmitter

\section{Test \#2}

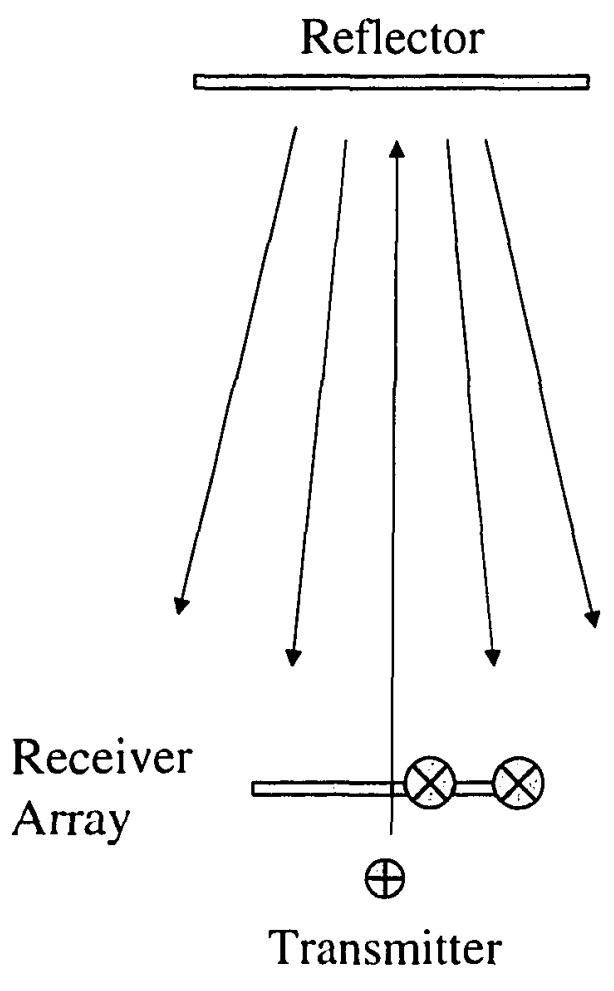

Figure 36. This diagram shows how the test should be conducted for multiple antenna emulation. In test session \#I, the signal going to the right hand side is captured by the receiver on the right. In test session \#2, the antenna array is moved to the left to capture the signal reflected to the left hand side.

only a single signal path. If the transmit power of the signal is large, it causes multi-path signals to be captured: therefore, the environment will not be reproducible from one capture session to the next. Finally, the data is encoded such that the sequences can be easily matched up in software. One method to match the data sequence is to start the capture first before turning on the transmitter. Therefore, one can match the two data sequences based on the changes in the output power at the receivers.

\subsubsection{Multi-path Emulation}


Using a similar idea as the multi-antenna emulation, one could emulate a multipath environment using superimposition, as shown in Figure 37. For this particular setup, one captures the transmitted data for the first path. Afterwards, the configuration of the reflectors can be changed and the signal capture sequence is re-run. Once both sets of data are available, the data can simply be summed together at the software level. The resulting signal is fed into the beam-former for further processing.

In order for this setup to work properly, several assumptions are made. First of all, the environment should be modified slightly for each capture session to ensure that the data captured for the different paths are uncorrelated. Secondly, the software can delay the captured data in order for a particular signal path to emulate latency spread. Finally, the transmit power of the signal source should be limited to ensure that a single path is captured during each trial. 


\section{Path \#1}

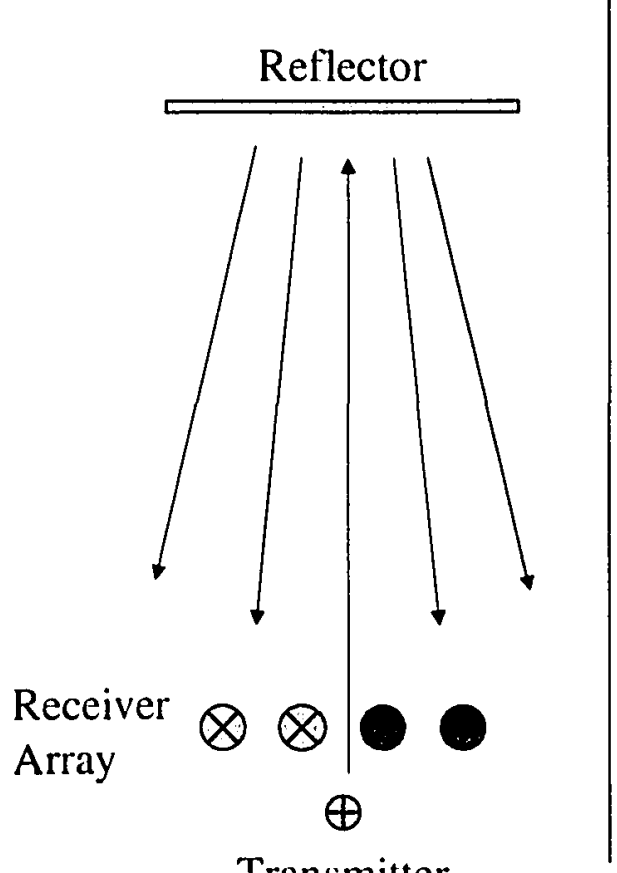

Transmitter
Path \#2

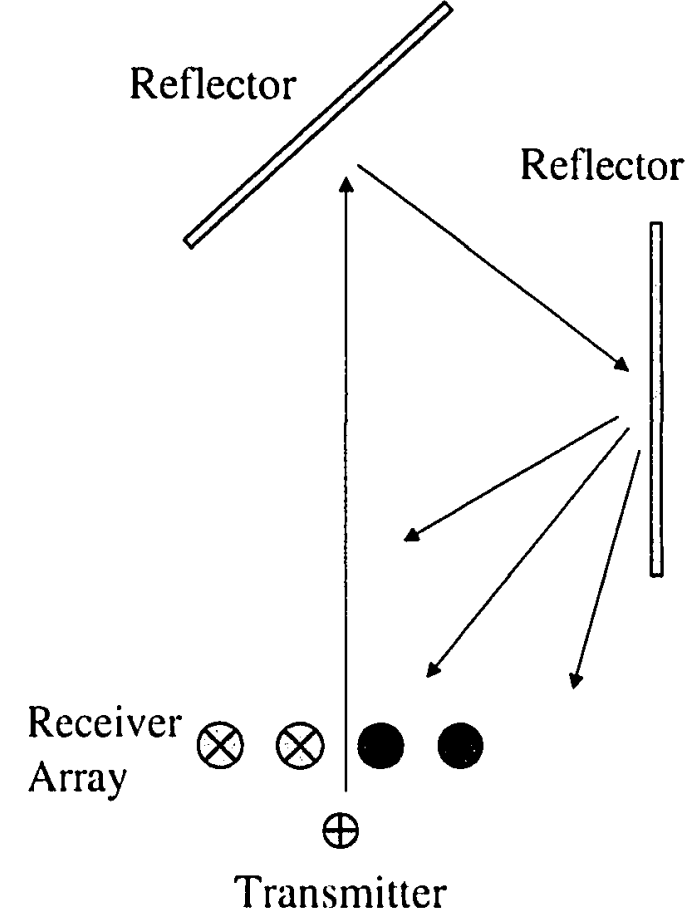

Figure 37. The diagram shows how the test should be conducted for multipath emulation. In test session \#I, a short delay spread channel is emulated. In the test session \#2, the environment is changed to create a different signal path. 


\section{Experimental Results}

All of the results presented in this chapter are based on data collected using the test system setup described in the previous chapter. There are two objectives, which the experiments attempt to demonstrate. The first objective is to repeat some of the results shown in chapter 3 -in particular, the system performance results caused by changing the various system parameters. The second objective is to use the real-life data to demonstrate that the error feedback signal delay compensation system performs better than the uncompensated combined beam-former and equalizer system, in terms of BER as well as convergence speed. This chapter is divided into three sections. The first section describes the setup of the experiments and what kind of data is captured. The second section will present the convergence speeds and BER performance for the various systems using the captured data. The final section will discuss the results presented in the second section.

\subsection{Experiment Setup}

In this section, the setup of the experiments is discussed. In the first part, the data-capturing environment is examined. In the second part, the procedures used to capture the data are discussed.

\subsubsection{Experiment Test Environment}

All of the experiments are will be performed in the room shown in Figure 38. The transmitting antenna is placed at the lower left corner of the room and the transmitted message is radiated in a forward direction. The receiving antennas are placed behind the 

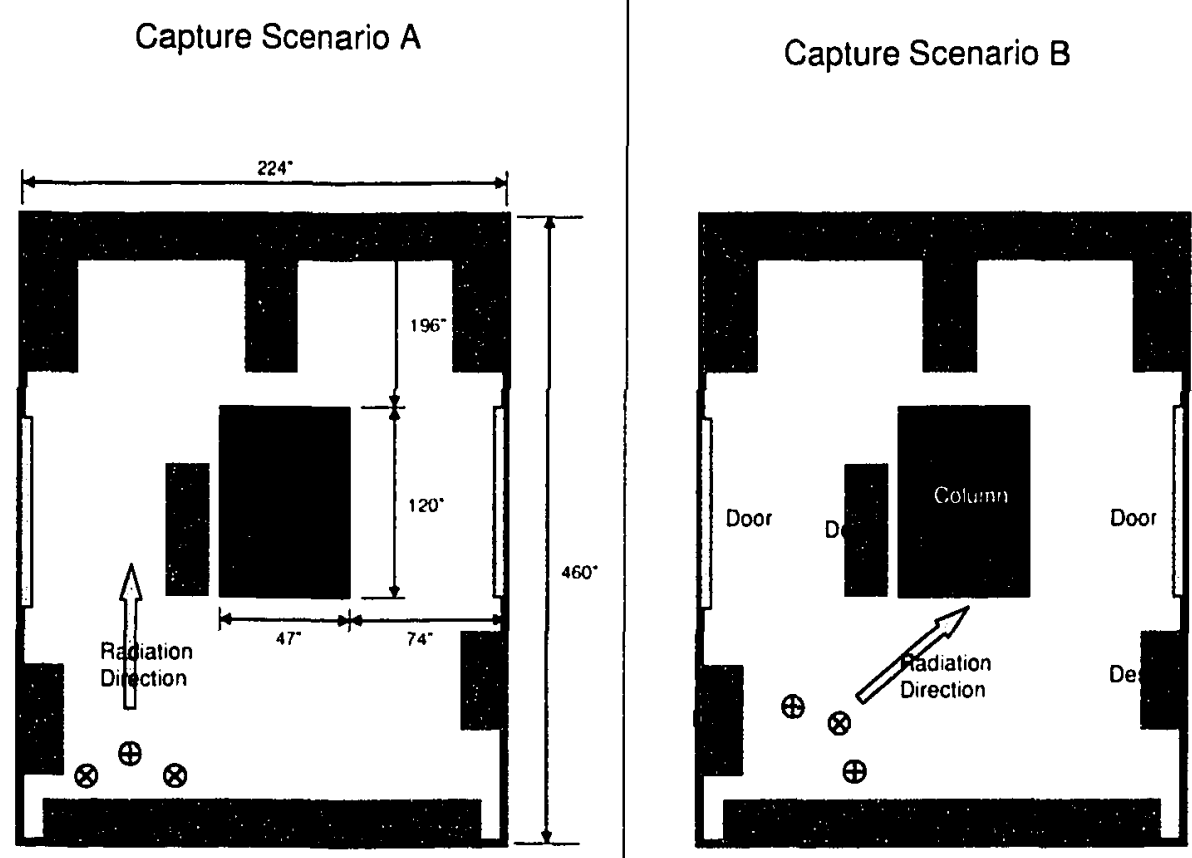

Figure 38. The layout and the dimension of the room in which the experiments are conducted are shown in the above figure; in addition, the two capture environments are also presented.

transmitting antenna, which will eliminate any line of sight signal paths. However, because the size of the room is relatively small, it is quite difficult to mimic a large path latency spread. Therefore, the multiple-path emulation technique described in the previous section is used to emulate a large delay spread channel. In order to change the characteristics of the channel, the antenna structure can simply be turned to face a different direction.

\subsubsection{Experiment Scenarios}

There are several channel environments in which the receiver system will be tested. For each channel environment, 2 seconds worth of data will be recorded for 3 


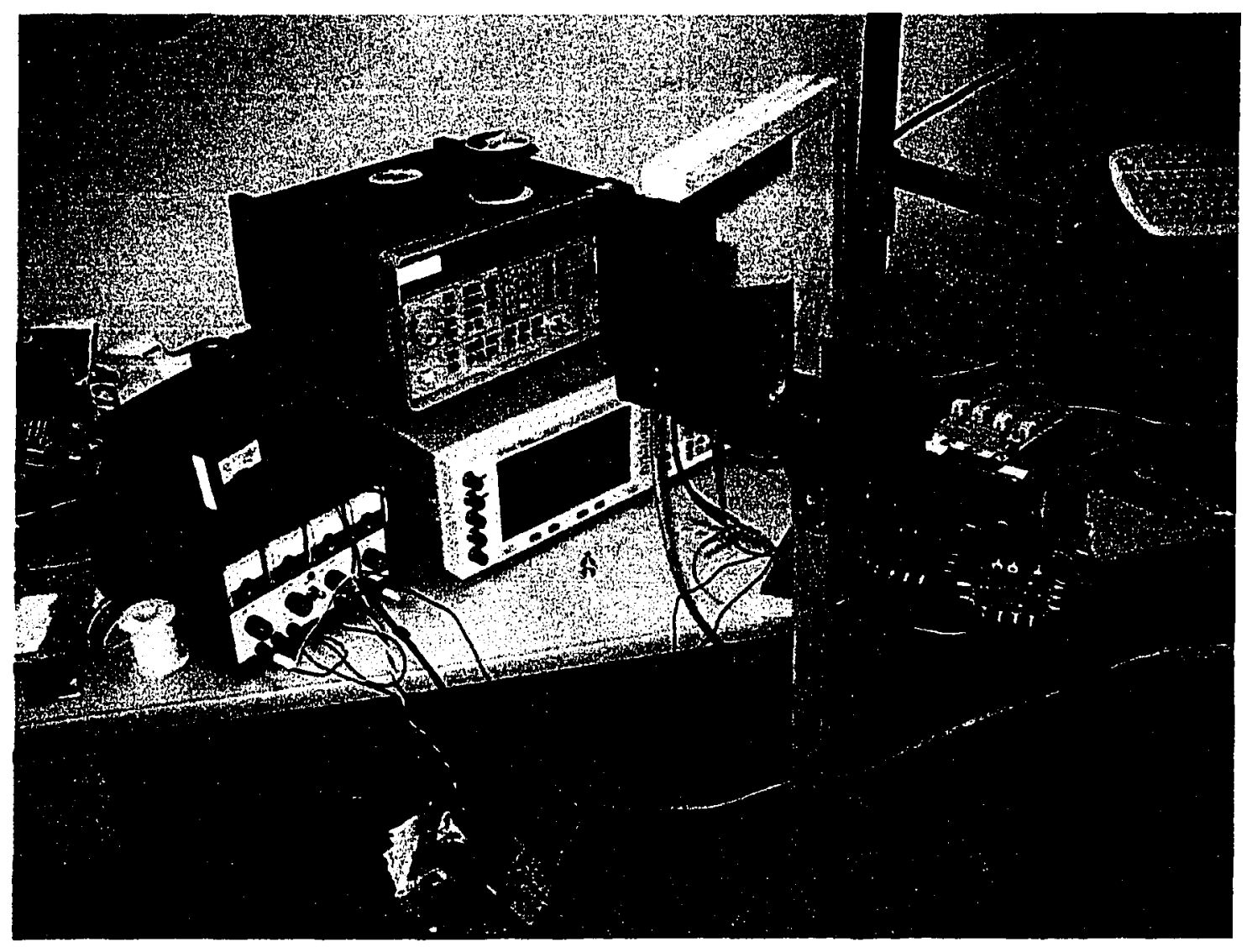

Figure 39. Photo of the capture system.

different trials. Captures in the same channel environment will be repeated for different levels of transmit power, which will vary from $5 \mathrm{dbm}$ to $-5 \mathrm{dbm}$ every $1 \mathrm{dbm}$ increments. The following is a list of the channel environments that the experiments try to reproduce:

1. A multiple data-path channel with a small latency spread between each signal path. Each of the receiver systems is assumed to have only four antennas. (The multiple antenna emulation technique described in the previous chapter is used.)

2. A multiple data-path channel with a large latency spread between each signal path. Each of the antenna systems is assumed to have two antennas. 


\subsection{Performance Comparison}

Using the captured data collected from each of the channel environments described above, the performances of different receiver designs are compared. Each system is judged based on the convergence time and the BER performance. In the first half of this section, we examine different combined beam-former and equalizer systems. In the second half, we present the performance gain offered by the error signal feedback delay compensation system.

\subsubsection{The Combined beam-former and equalizer System}

The effect of the different system parameters on the performance of the combined beam-former and equalizer system were examined in Chapter 3, using the simulation environment; this section attempts to reproduce some of the results observed in Chapter 3 , using the captured data. Different receiver systems with various values of $L_{H F}$ process the captured data; the system parameters for all the receiver systems are described in Table 7. Due to the synchronization limitations of the capture system, only the parameter $L_{k r}$ for the beam-former is discussed. The parameter $L_{r r}$ must have the same length as the super-frame: otherwise, the synchronization of the system will be broken. The various receivers will be evaluated, based on the convergence speed and BER in two different channel environments: the channel with little delay spread. and the channel with a larger delay spread. 
Table 7 The following table shows the value for each of the transmission system parameters.

\begin{tabular}{|c|c|c|}
\hline $\begin{array}{l}\text { Parameter } \\
\text { Name }\end{array}$ & Value & Description \\
\hline$N_{A}$ & 2,4 & $\begin{array}{l}\text { The number of antennas for the receiver will change } \\
\text { from channel to channel. For the channel with little } \\
\text { delay spread, four antennas are used, and for the } \\
\text { channel with a large delay spread, two antennas are } \\
\text { used. }\end{array}$ \\
\hline$\eta$ & 8 & $\begin{array}{l}\text { The number of samples per symbols is set to } 8 \text { to limit } \\
\text { the required length of the beam-former and the } \\
\text { equalizer. }\end{array}$ \\
\hline$\chi$ & N/A & $\begin{array}{l}\text { The down-sampling factor, or the fractional step size, } \\
\text { of the matched filter is one of the parameters being } \\
\text { studied. In typical simulations, } \chi \text { is set to } 4 \text {. }\end{array}$ \\
\hline$\beta$ & N/A & $\begin{array}{l}\text { The down-sampling rate of the feedforward filter, } \beta \text {, } \\
\text { varies from simulation to simulation. However, } \beta \chi \text { is } \\
\text { constrained to } 8 \text {. }\end{array}$ \\
\hline$T$ & 4 us & $\begin{array}{l}\text { The symbol rate of the system is set to } 250 \mathrm{ksymbols} / \mathrm{s} \\
\text { to mimic the symbol rate of the experimental capture } \\
\text { system described in section } 5 \text {. }\end{array}$ \\
\hline$\xi$ & 1 & $\begin{array}{l}\text { The update rate of the beam-former LMS algorithm is } \\
\text { set to } 1 \text { to improve the convergence characteristics of } \\
\text { the system. }\end{array}$ \\
\hline$f_{\text {. }}$ & $2.1875 \mathrm{GHz}$ & $\begin{array}{l}\text { The carrier frequency of the system is set to } 2.1875 \\
\mathrm{GHz} \text { to mimic the carrier frequency used in the } \\
\text { experimental capture system described in section } 5 \text {. }\end{array}$ \\
\hline$\alpha$ & 1 & $\begin{array}{l}\text { Because bandwidth is not an issue in the capture } \\
\text { environment, the maximum bandwidth is used. }\end{array}$ \\
\hline$L_{B r}$ & N/A & The length of the beam-former will vary from 1 to 16 \\
\hline$L_{r F}$ & 96 & This is set to 96 for synchronization results. \\
\hline$L_{R K}$ & 8 & The length of the feedback filter is set to 8 . \\
\hline
\end{tabular}



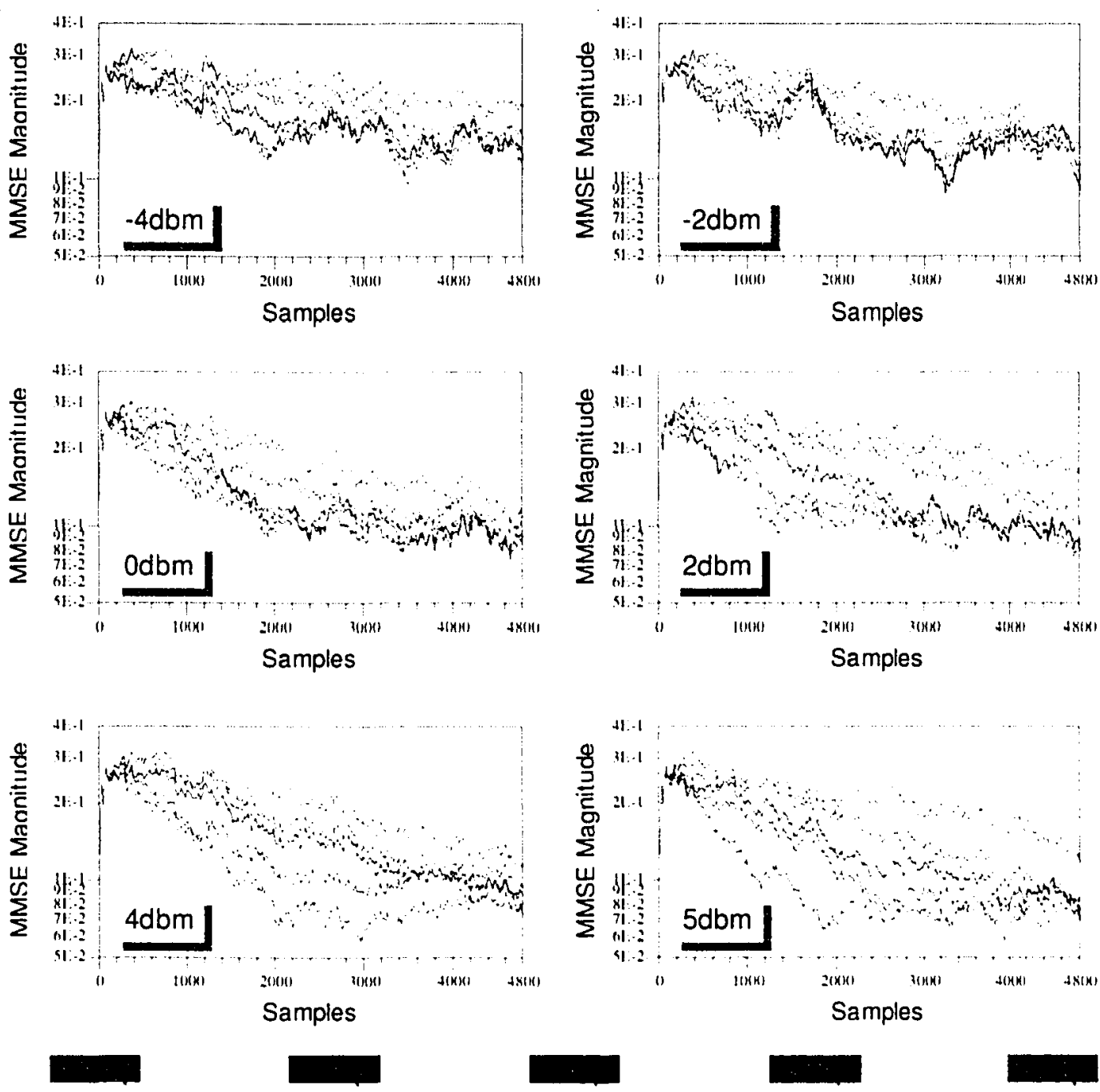

Figure 40. This diagrams show the MSE magnitudes for all of the different receivers, when the transmit power varies from $-5 \mathrm{dBm}$ to 5 $\mathrm{dBm}$ in a channel with little delay spread.

\subsubsection{Convergence Time}

The convergence time is measured based on the speed of the LMS algorithm to reach a steady-state solution. It is obvious from Figure 40 and Figure $4 \mathrm{l}$ that none of the receiver systems really converge until the transmit power reaches $0 \mathrm{dbm}$; from $0 \mathrm{dbm}$ on, 
both figures show that the higher number of taps in the beam-former has resulted in a faster convergence speed, i.e., it takes less time for the LMS algorithm to reach the steady-state. However, it is also obvious that, for both channel conditions, when the transmit power is high, the number of taps in the beam-former no longer plays a critical role in the convergence speed of the system. This, in general, matches the results observed in the simulation presented in Chapter 3 . When comparing the convergence speed under different channel conditions, another observation can be made. It is clear from comparing Figure 40 and Figure 41 that the combined beam-former and equalizer performs better when the delay spread in the channel is small. This is because none of the receivers used in the experiment has the error signal delay compensation system; consequently, when the delay spread is large, the optimal error signal feedback might vary from trial to trial. Thus, the overall performance is reduced. In addition, for the channel with little spread, there are 4 antennas attached to the receivers; thus, the average noise coming out of the beam-former is lower. 

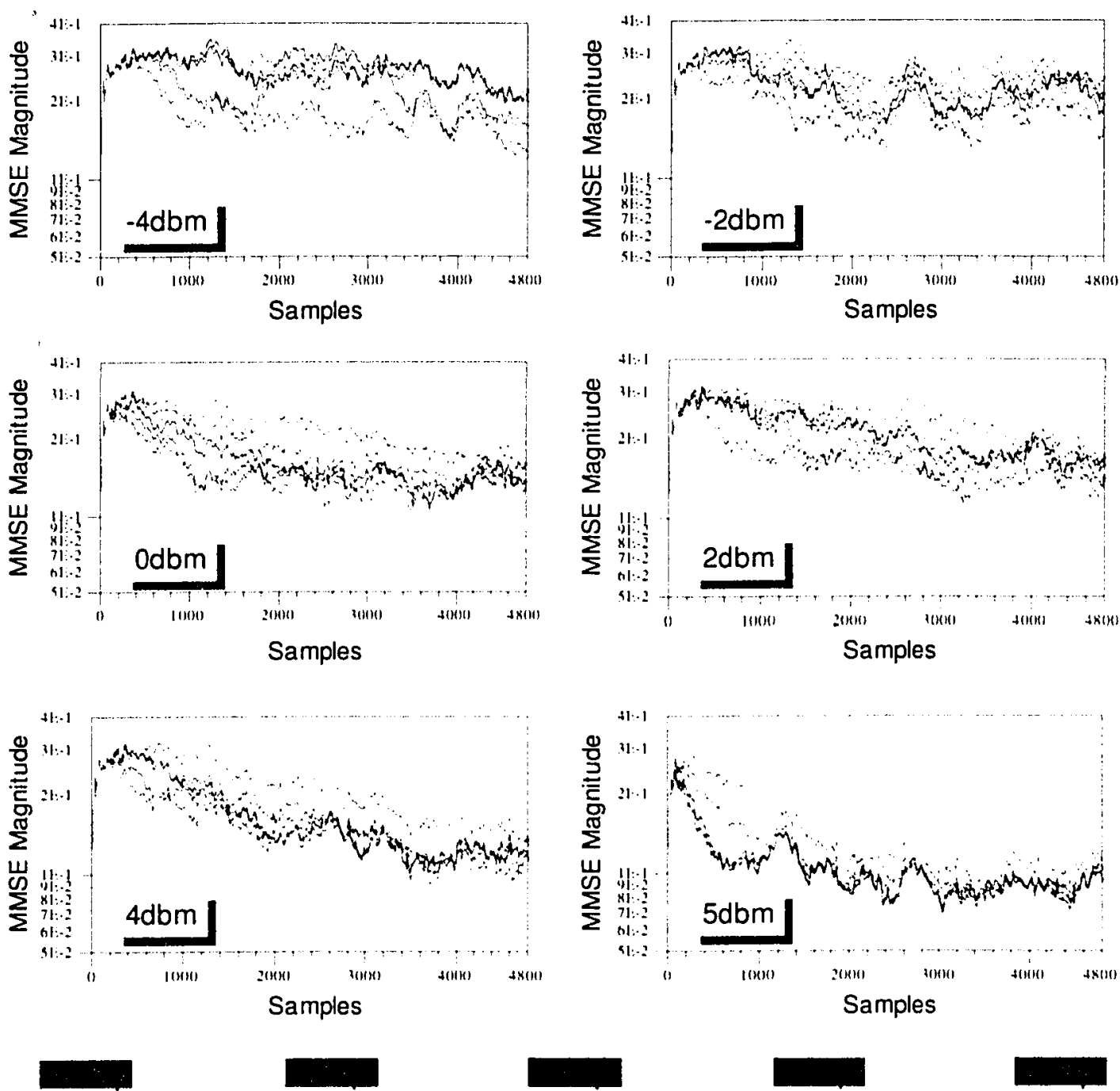

Figure 41 . This diagrams show the MSE magnitudes for all of the different receivers when the transmil power varies from $-4 \mathrm{dBm}$ to 5 $\mathrm{dBm}$ in a multi-path channel. 


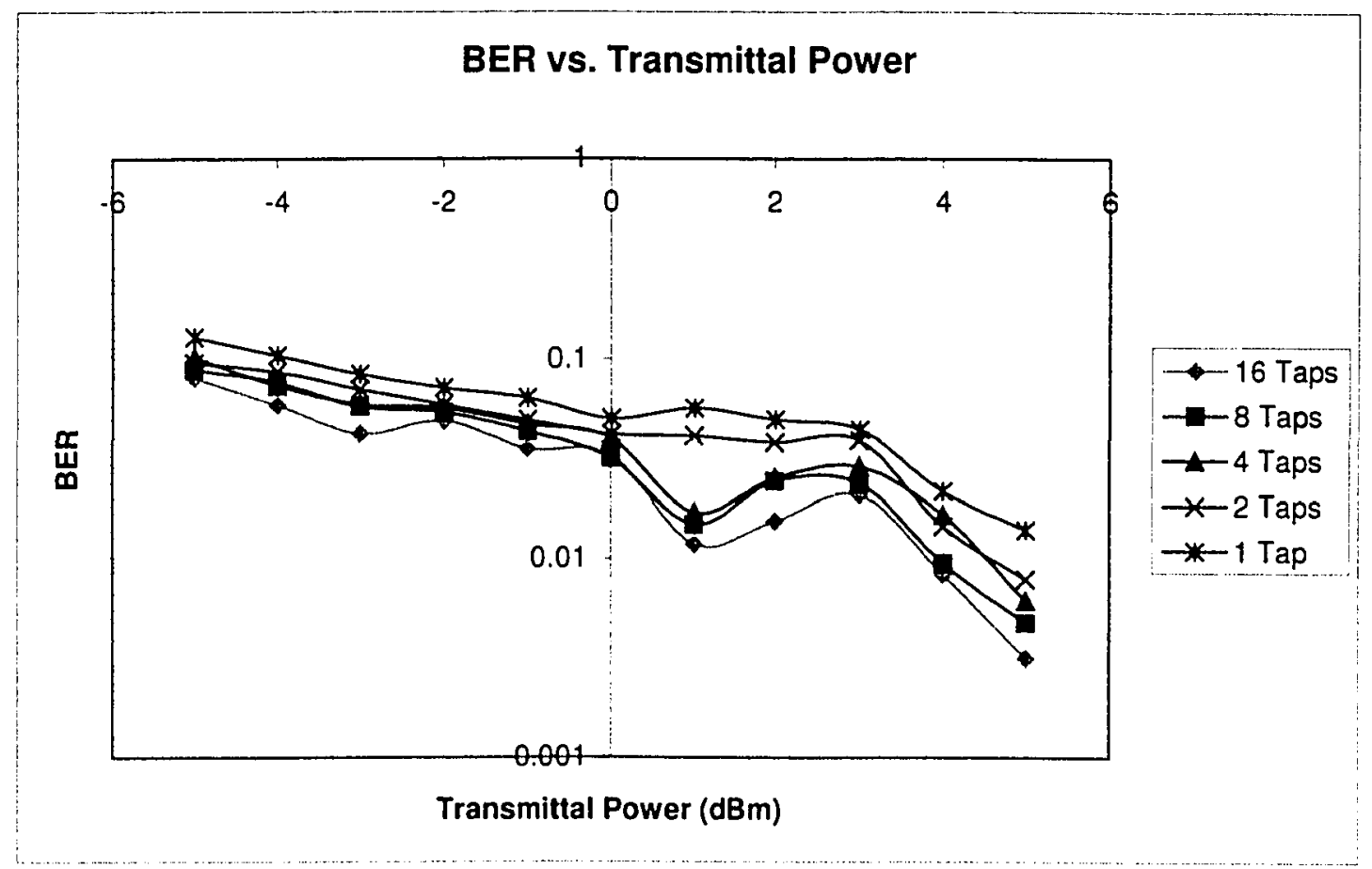

Figure 42. This diagram shows the average BER performance for a channel with little delay spread.

\subsubsection{BER}

If one were to also examine the BER performance of the different receiver systems, it is clear from Figure 42 and Figure 43 that as the parameter $L_{\mu t}$ increases, the BER generally improves slightly for both channel conditions, i.e., the channel with little delay spread and the channel with a large delay spread. Furthermore, the BER improvement diminishes as $L_{k r}$ increases. This behavior is consistent with the results obtained from the simulation in Chapter 3. Similar to the convergence speed, it is also obvious from Figure 42 and Figure 43 that all systems performs better in the channel with little delay spread. 


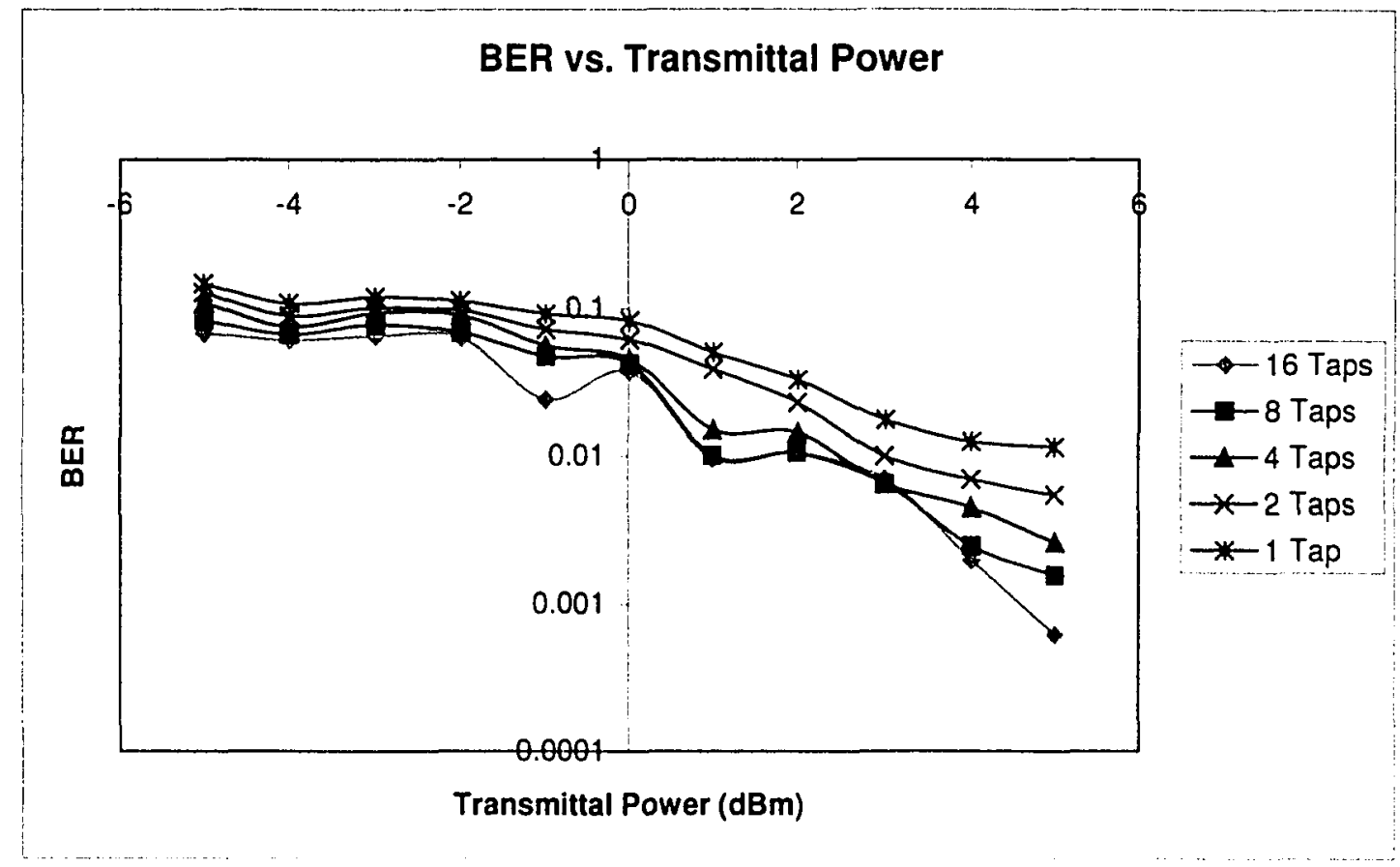

Figure 43. This diagram shows the average BER performance for a channel with a large delay spread

\subsubsection{Variable Delay Error Signal Feedback Compensation System}

One of the problems with the combined beam-former and equalizer system is that the performance of the system depends on the latency of the crror signal going to the beam-former. In the previous section, it has been shown in simulation that by compensating for the latency of the error signal introduced by the feedforward filter, both the resulting convergence time and the BER will to improve. In this section. the performances of the two receivers, one with the error signal latency compensation and the other one without the compensation, will be compared in two different channel environments: a channel with little delay spread, and a channel with a large delay spread. The two receivers are identical, except for the error feedback delay compensation system, 
and their system parameters are described in Table 8. The two receivers will be evaluated based on the convergence time and BER in both channel conditions.

Table 8 The following table shows the value for each of the transmission system parameters.

\begin{tabular}{|c|c|c|}
\hline $\begin{array}{l}\text { Parameter } \\
\text { Name }\end{array}$ & Value & Description \\
\hline$N_{A}$ & 2,4 & $\begin{array}{l}\text { The number of antennas for the receiver will change } \\
\text { from channel to channel. For the channel with little } \\
\text { delay spread, four antennas are used and for channel } \\
\text { with a large delay spread, two antennas are used. }\end{array}$ \\
\hline$\eta$ & 8 & $\begin{array}{l}\text { The number of samples per symbol is set to } 8 \text { to limit } \\
\text { the required length of the beam-former and the } \\
\text { equalizer. }\end{array}$ \\
\hline$\chi$ & N/A & $\begin{array}{l}\text { The down-sampling factor, or the fractional step size, } \\
\text { of the matched filter is one of the parameters being } \\
\text { studied. In typical simulations, } \chi \text { is set to } 4 \text {. }\end{array}$ \\
\hline$\beta$ & N/A & $\begin{array}{l}\text { The down-sampling rate of the feedforward filter, } \beta \text {, } \\
\text { varies from simulation to simulation. However, } \beta \chi \text { is } \\
\text { constrained to } 8 \text {. }\end{array}$ \\
\hline$T$ & 4 us & $\begin{array}{l}\text { The symbol rate of the system is set to } 250 \mathrm{ksymbols} / \mathrm{s} \\
\text { to mimic the symbol rate of the experimental capture } \\
\text { system described in section } 5 \text {. }\end{array}$ \\
\hline$\xi$ & 1 & $\begin{array}{l}\text { The update rate of the beam-former LMS algorithm is } \\
\text { set to } 1 \text { to improve the convergence characteristics of } \\
\text { the system. }\end{array}$ \\
\hline$f_{c}$ & $2.1875 \mathrm{GHz}$ & $\begin{array}{l}\text { The carrier frequency of the system is set to } 2.1875 \\
\mathrm{GHz} \text { to mimic the carrier frequency used in the } \\
\text { experimental capture system described in section } 5 \text {. }\end{array}$ \\
\hline$\alpha$ & 1 & $\begin{array}{l}\text { Because bandwidth is not an issue in the capture } \\
\text { environment, the maximum bandwidth is used. }\end{array}$ \\
\hline$L_{B r}$ & 16 & $\begin{array}{l}\text { The length of the beam-former is set to } 16 \text { for both } \\
\text { receivers }\end{array}$ \\
\hline$L_{r k}$ & 96 & This is set to 96 for synchronization results. \\
\hline$L_{B F}$ & 8 & The length of the feedback filter is set to 8 . \\
\hline
\end{tabular}



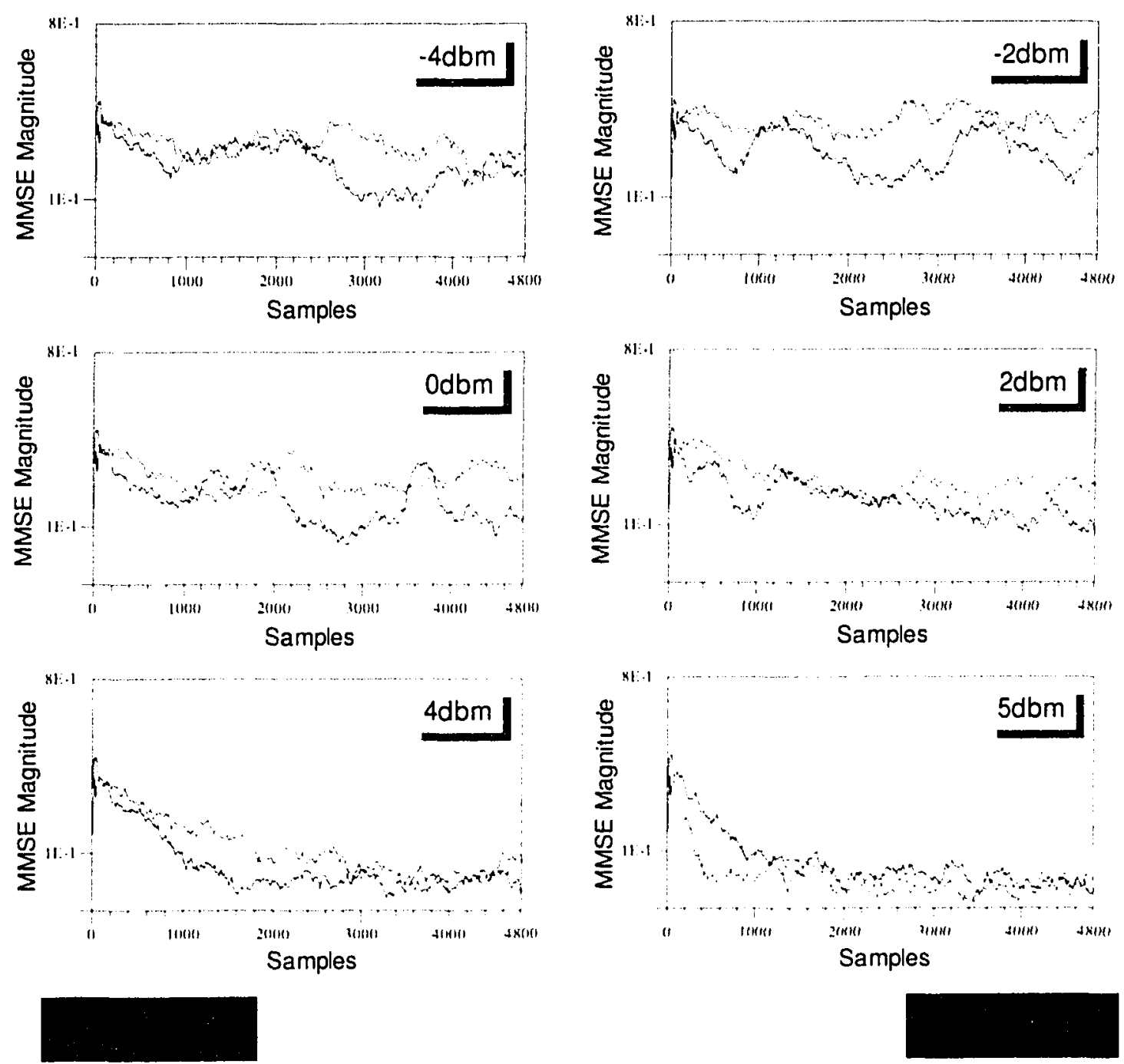

Figure 44 The diagrams show the MSE magnitudes for both the compensated and the uncompensated system when the transmit power varies from $-4 \mathrm{dBm}$ to $5 \mathrm{dBm}$ in a channel with little delay spread.

\subsubsection{Convergence Time}

As shown in Figure 44 and, Figure 45 the compensated system performs slightly better than the uncompensated system in terms of convergence time for both channel conditions, in most cases. In fact, for low transmit power, the compensated system is 

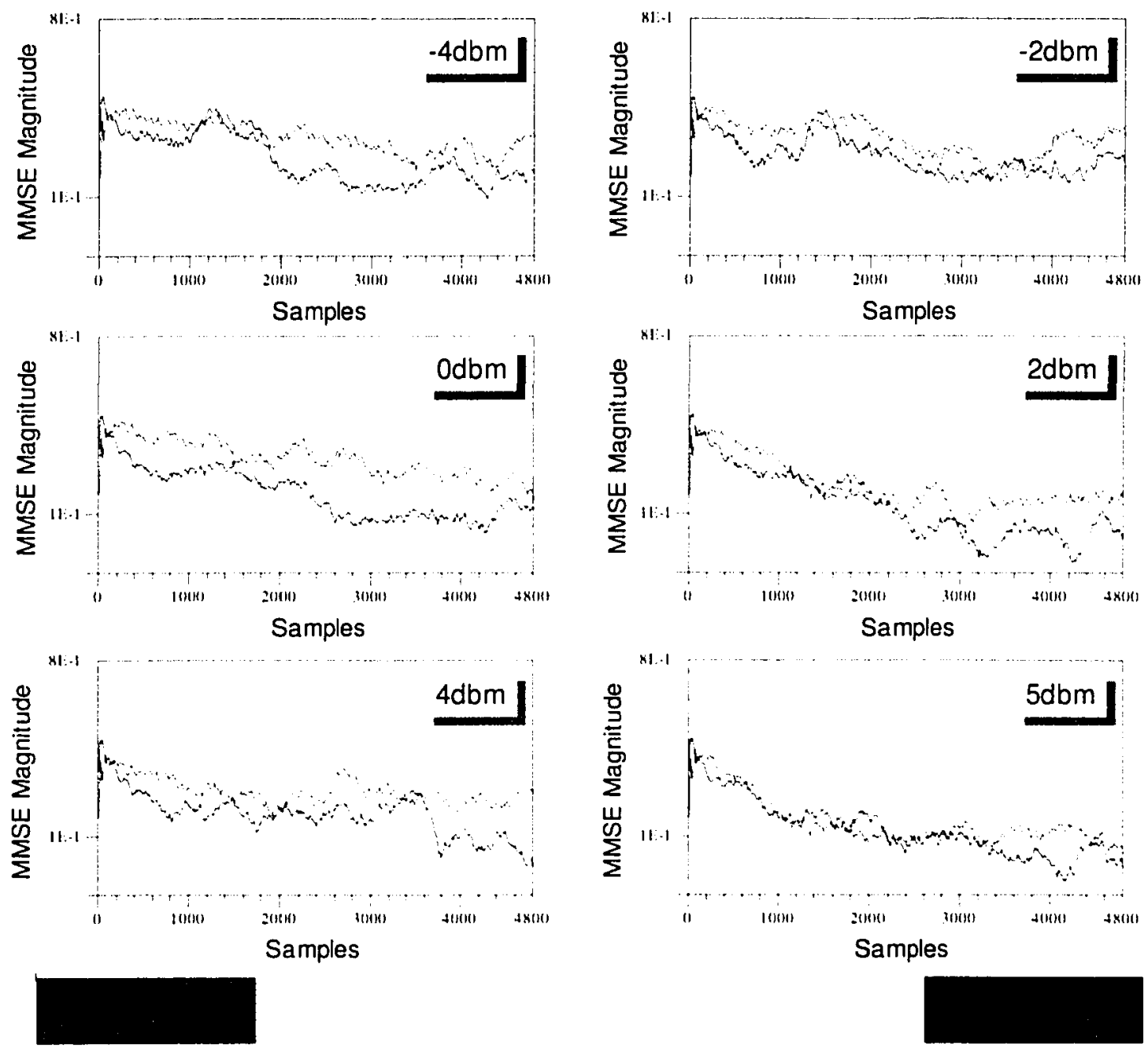

Figure 45. The diagrams show the MSE magnitude for both the compensated and the uncompensated system when the transmit power varies from $-4 \mathrm{dBm}$ to $5 \mathrm{dBm}$ in a large delay spread channel.

able to reach a lower MSE level faster than the uncompensated system. For high transmit power, i.e. when the transmit is $5 \mathrm{dbm}$, the convergence time improvement diminishes and, in some cases, the uncompensated system out-performs the compensated system.

This observation can be explained by examining the structure of the error compensation system. The latency compensated system requires time for the error latency to reach the optimal value, during which time the state of the LMS algorithm will 


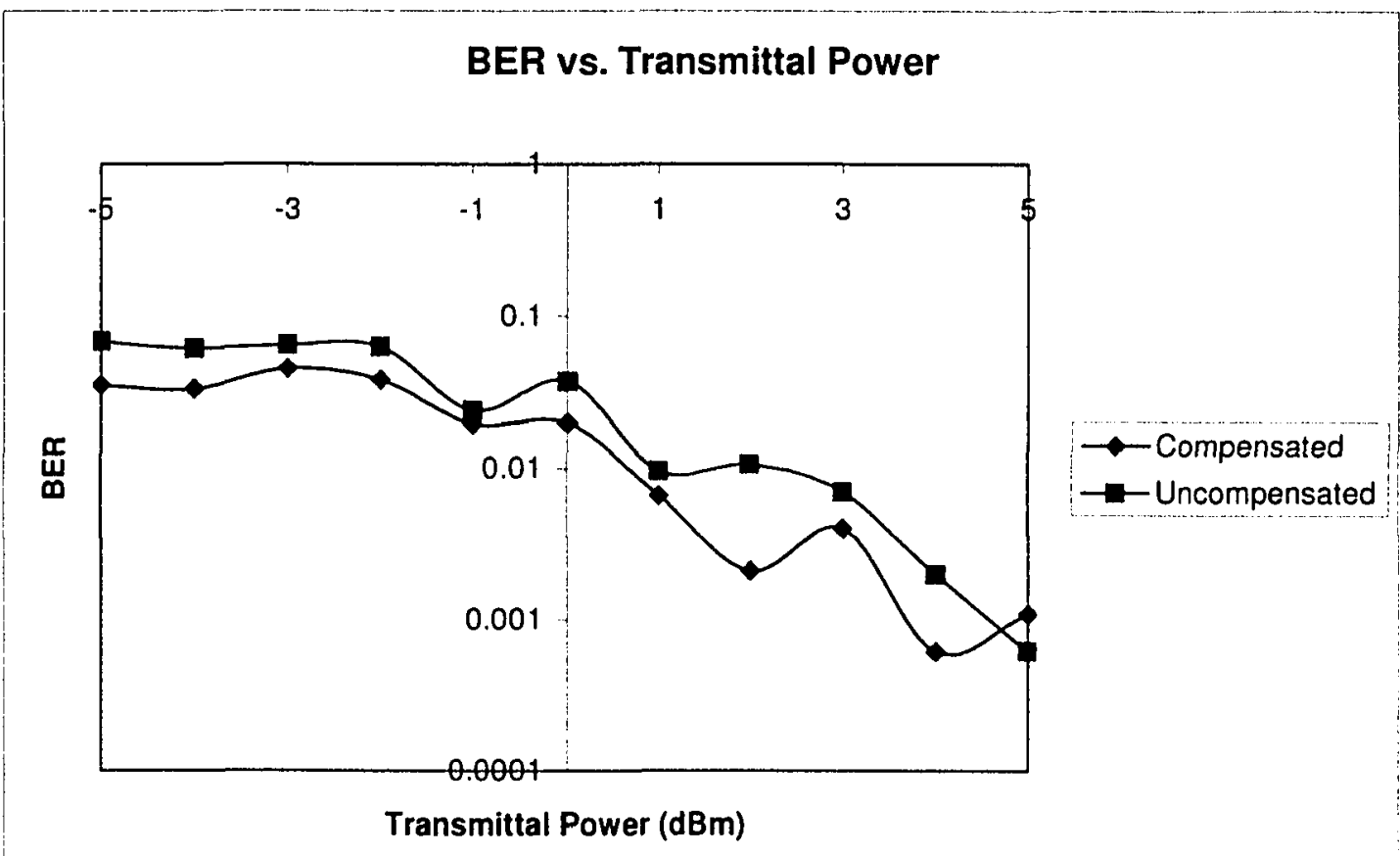

Figure 46. The average BER performance for a channel with little delay spread shows that the compensated system is only marginally better than the uncompensated.

be perturbed as the error signal latency changes. Hence, the convergence time of the system might be impacted adversely.

\subsubsection{BER}

The BER performance of the compensated receiver system is better than that of the uncompensated system for both channels. Figure 46 and Figure 47 show the performance difference between the two systems. Even though Figure 46 has shown that the compensated receiver performs better than the uncompensated receiver, the difference is only marginal, i.e., less than $0.5 \mathrm{~dB}$ of transmit power different. Given the additional computational power required to calculate the proper error signal latency, and the marginal performance improvement, the error compensation scheme might not be 


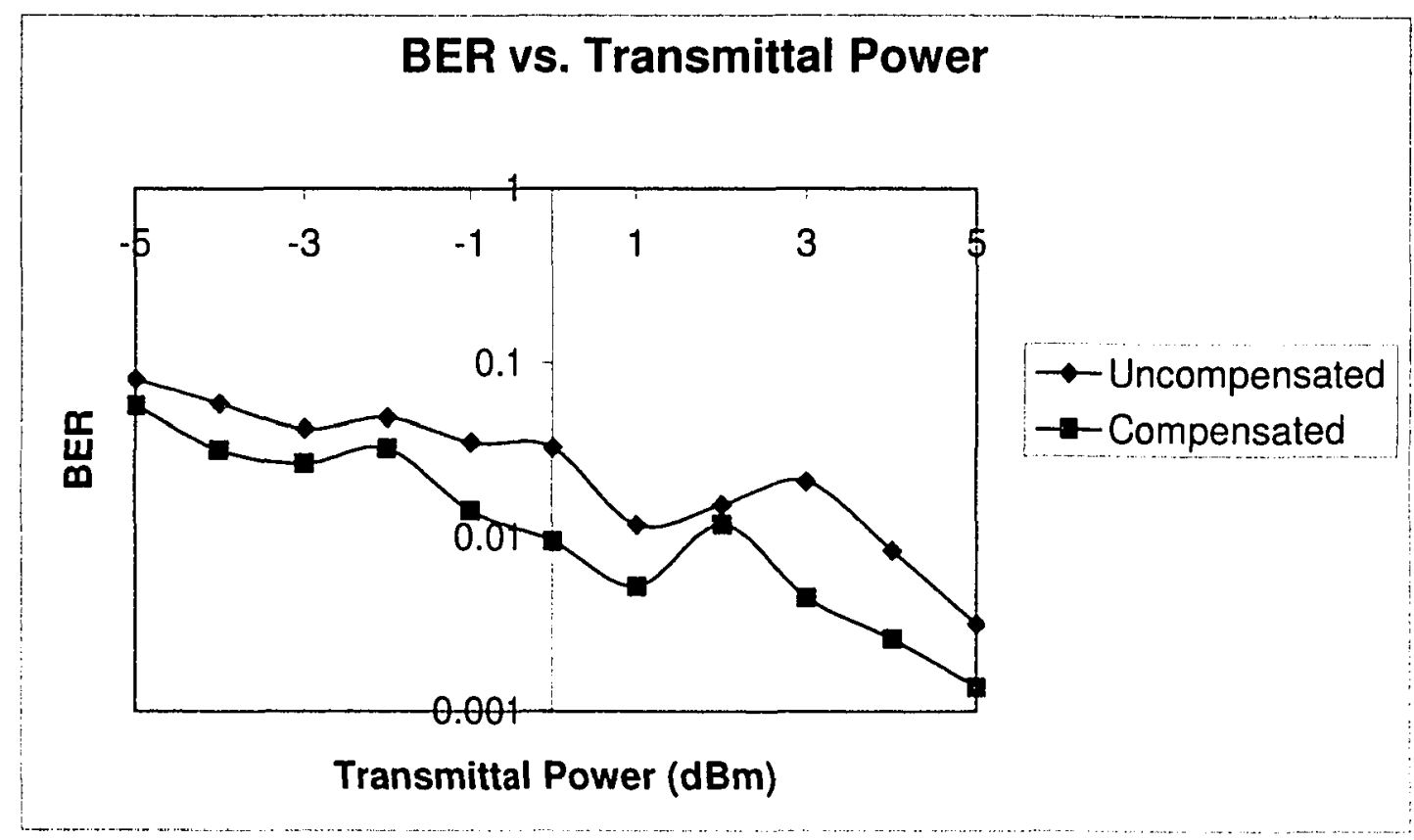

Figure 47. The average BER performance for a channel with a large delay spread is slightly better for the compensated system than the uncompensated system.

suitable for a receiver system with low power and computational requirements. In simulation, the performance improvement is a bit more significant, which might be because the latency spread is larger in the simulations. Figure 47 shows the performance gain of the compensated system in a multi-path channel with a larger delay spread and the performance difference between the compensated system and the uncompensated system is greater in a channel with a larger delay spread. The performance gain varies from $1 \mathrm{dBm}$ to $1.5 \mathrm{dBm}$ of transmit power, which is better than the mere $0.5 \mathrm{~dB}$ gain in a channel with little delay spread. Nonetheless, it is still not as significant as the performance gain demonstrated in simulation. 


\subsection{Discussion}

The following section will discuss and summarize the results presented in the previous section. The first part of this section will examine some of the problems observed in the signal capture system. The second part will discuss the results presented for the parameter sweeping of the combined beam-former and equalizer system. The final part of this section will examine the results presented in the error feedback delay compensation system.

\subsubsection{The Signal Capture System}

Although the signal capture system is able to successfully capture some useful data for the experiments, the design of the system and the procedure used to capture the data suffers from a number of problems. First of all, the signal capture system is built using discrete modules connected together using SMA connectors and cables. As a result, far too much noise is introduced into the capture system, due to various electrical problems. Secondly, there is no carrier recovery system in the receiver's front-end; as a result, the carrier will need to be conducted using software. The problem related to current setup for carrier recovery is explained in Appendix A. Finally, during the signal capturing process, the control of the channel environment is very limited. Because there are people entering and exiting the room, the BER curve presented in this section doesn't follow the classical shape of the simulated BER curve. There are times in which a lower SNR will perform better than a higher SNR. 


\subsubsection{The Combined beam-former and equalizer System}

The result obtained from the real-life capture data is generally consistent with the simulation results shown in Chapter 3. For different transmit power and channel conditions, the receiver with a longer beam-former seems to perform better in terms of convergence speeds and BER. Furthermore, the convergence speed and BER advantage diminishes as the length of the beam-former increases. This behavior is particularly obvious in the larger delay spread channel.

\subsubsection{The Variable Delay Error Signal Feedback Compensation System}

The compensated system offers performance gains over the uncompensated system and its behavior is consistent with the simulated results obtained in Chapter 4 . However, in both Figure 46 and Figure 47, the performance gain over the uncompensated system is very limited, and the performance advantage diminishes as the transmit increases. This is different from the result observed in the simulation.

This performance degradation can be explained if the structure used for compensating the error feedback delay is examined. The slower convergence time at the high SNR and low delay spread is because the compensated system takes time to calculate the proper error latency and, during this time, the system is not stabilized: hence, the compensated system does not converge as quickly. In contrast, the uncompensated system is able to move quickly to a solution and, although it might not be the most optimal solution, the uncompensated system reaches the steady state much more quickly. This effect reduces the performance gain offered by the latency compensation. 
High Delay Spread
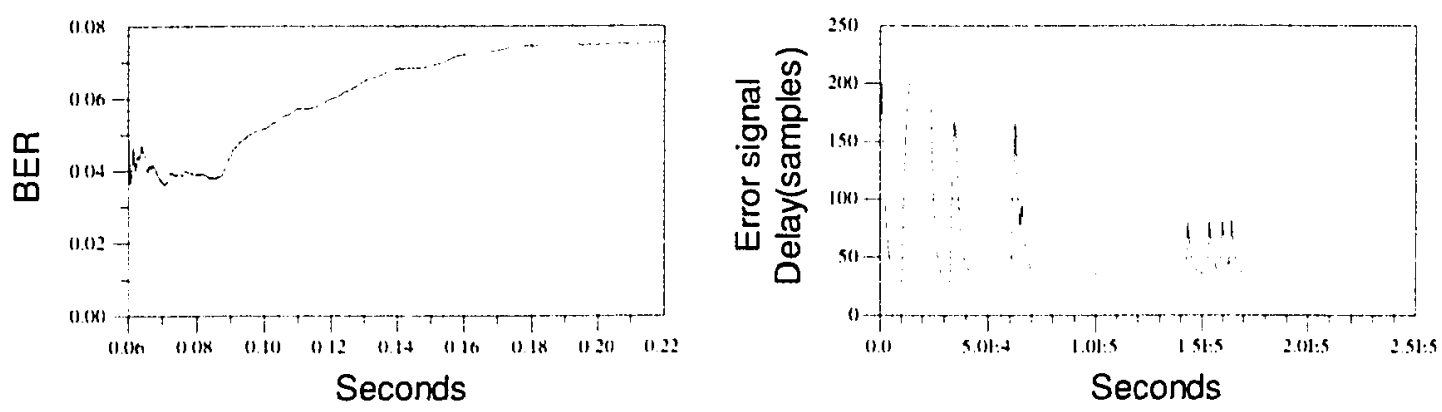

\section{Low Delay Spread}
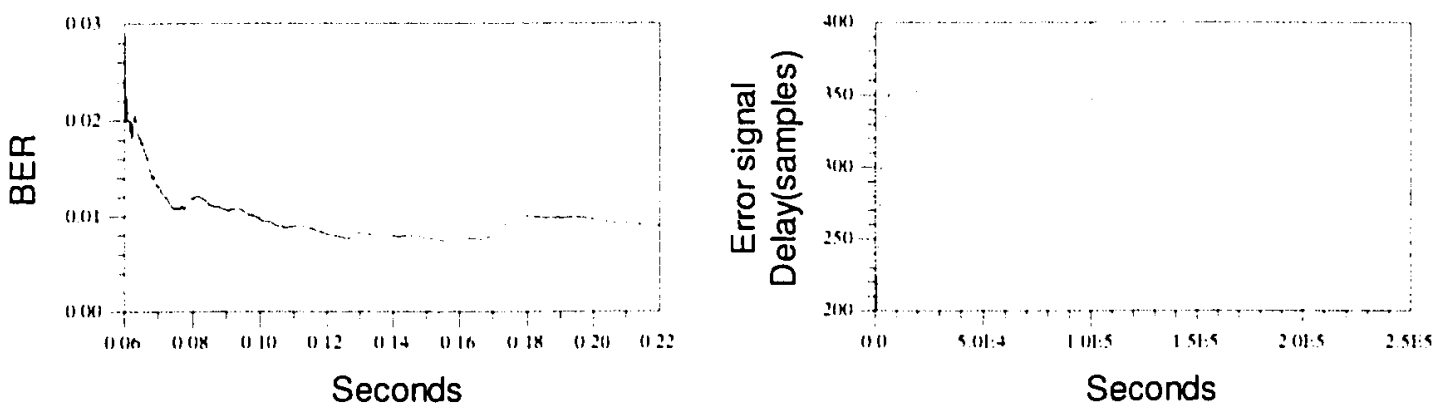

Figure 48. The diagram shows the output of the latency calculator and the cumulative BER over time.

In addition, oscillation in the delay value can sometimes change the stability point of the system; hence, it reduces the actual performance of the system. This effect is particular evident when there are two paths with large delay differences and almost the same power level. (This is highly unlikely in real life because the extra distance that the signal would need to travel would reduce the signal power at the receiver significantly. Therefore, the shorter path usually has higher signal power at the receiver.) Figure 48 shows the BER output and the latency of the error feedback signal of a compensated receiver. In Figure 48, it is very clear that the oscillation of the error signal latency is a significant problem, especially for the channel with a large delay spread, because it causes the BER to rise continuously, as the LMS algorithm becomes unstable. In the 
case of the low delay spread, the error signal doesn't oscillate as much; hence, the BER reaches a steady state.

There are several solutions that can address this problem. First of all, one could monitor the BER of the system; if the BER remains below a certain preset threshold, the latency compensation system can be disabled altogether. Thus, the oscillation can be prevented. Secondly, the compensation system can increase the time between successive error latency updates as the MSE magnitude decreases. 


\section{Conclusion and Future Work}

\subsection{Conclusions}

Traditionally, the error signal feeding the adaptive algorithm in the beam-former can be calculated using the direct output of the beam-former; however, some systems use the error signal generated at the output of the equalizer to update the beam-formers. Because of the larger number of taps in the equalizer, systems using the combined beamformer and equalizer architecture tend to have a larger acquisition range. In addition, because the equalizer is able to perform temporal filtering, the combined beam-former and equalizer architecture is able to combine signals from multiple signal paths with large delay spreads.

In a combined beam-former and equalizer architecture, there are various different parameters, such as the number of taps in the beam-former, the number of taps in the equalizer, and the fractional step size of the equalizer, all of which will affect the performance of the receiver. For the LMS based adaptive beam-former, this thesis has shown that increasing the length of the beam-former. $L_{H F}$, and the length of the forward filter of the equalizer, $L_{r+}$, will improve the BER performance and the convergence time of the system. The same effect on the receiver performance can also be observed if the fractional step ratio of the forward equalizer decreases. However, all of these changes will increase the computational resources required of the receiver. Moreover, asymptotic behavior can be observed as each of the parameters increases or decreases. Furthermore, this thesis has shown that a higher number of taps in the equalizer proves to be more 
effective in reducing BER than increasing the number of taps in the beam-former. Therefore, one should keep the number of taps in the beam-former to around 2 symbollengths; meanwhile, the number of taps in the equalizer should be of the same length as the impulse response of the channel.

The combined beam-former and equalizer architecture offers a number of advantages over the traditional design: it can combine signals with different latency to enhance the SNR and has a wider acquisition range because of the higher number of taps in the equalizer. However, the combined architecture suffers from a number of problems: sensitivity to the magnitude of the error signal, slow convergence speed, and variable error feedback latency. The first two problems are inherent in the architecture and difficult to mitigate. However, the variable error feedback latency can easily be fixed by compensating the delay dynamically.

This thesis has shown that the latency of the error signal is related to the tap weights of the forward filter of the equalizer. Consequently, the latency of the error signal can be determined by examining the weights of the taps in the equalizer. The equalizer tap with the highest value indicates that the received signal has the highest correlation with the desired signal and one can calculate the latency of the error signal. $d_{b f}$, by subtracting the index value of the highest magnitude tap from the total number of taps in the equalizer. There are two different architectures that can be used to compensate for the variable error signal feedback delay. The first architecture uses a fixed error signal feedback delay, $d_{b f}$, but adjusts the latency of the desired signal input. It has been found in simulations that this type of design provides only a slight improvement over the conventional design. The second architecture adjusts the latency 
of the error signal going to the beam-former directly. This type of system provides real performance in simulation and, as a side effect, the convergence time of the system also improves when the SNR is low. A variant of the system adjusts the error delay constant of the beam-former. By adjusting the error delay constant directly, the performance gain obtained is the same or better than the systems, which adjust the latency of the feedback error signal; furthermore, the memory requirement for this type of system is lower.

After illustrating the effectiveness of the compensation systems in simulation, a signal capture system was built to run similar tests, using real-life data. Several scenarios were run: a 4-antenna receiver with a low delay spread, and a 2-antenna receiver with a large delay spread. Using the captured data, this thesis has repeated some of the parameter sweeping experiments, and the results obtained for sweeping the length of the beam-former, $L_{B F}$, from 1 to 16 were consistent with the simulated results presented in Chapter 3. This thesis has also shown that the longer the beam-former, the faster the system will converge and the better the resulting BER performance will be for both the channel with little delay spread and the channel with a large delay spread. Moreover, the improvements in both the BER and convergence time diminishes as the SNR increases and $L_{H F}$ increases.

Using the same set of capture data, this thesis has demonstrated that both the convergence speed and the BER in the error feedback signal delay compensated system perform better than in the uncompensated system. This is consistent with the conclusion drawn from the simulated data. However, the performance gain obtained from the compensation system is marginal in most cases. Although the BER improvement is much more significant for a channel with a large delay spread, the complexity of the 
operation and the computational resources required might outweigh the benefits obtained from the proposed system.

\subsection{Future Work}

Although the signal capture system has successfully captured the transmitted data, the design of the signal capture system suffers from a number of problems. First of all, many of the discrete modules used in the system are simply connected using SMA connecters and cables: consequently, various noise sources are introduced into the system. Secondly, there is no carrier recovery built into the receiver's front end; as a result, the carrier removal process has to be done with software. Appendix A has given a detailed discussion on the problems introduced by the lack of carrier recovery and some of their potential solutions. For the future capture system, a single PCB should be built, which will contain all of the receiver front-ends with carrier recovery as well as A/D converters. The single PCB will be plugged directly into the PC to stream the antenna data directly into the PC. As a result, better quality data can be obtained.

Although the error signal latency compensated system improves the resulting BER of the receiver, this system suffers from stability problems when there are two signal paths with large temporal differences but similar signal strengths. As a result, there will be two equal magnitude taps in the equalizer and random noise can cause the delay processor to flip-flop between the two values. As the error feedback signal latency value changes, the receiver will become unstable for a brief period of time. This problem is clearly demonstrated in the channel with a large delay spread using real data. Therefore, further work is required to build a better-designed delay calculator to fix this problem; for instance, the delay processor will only update its calculation if the BER 
exceeds certain pre-set thresholds. Alternatively, the compensation system can increase the time between successive error latency updates as the BER decreases. 


\section{A. Appendix - Carrier Recovery System}

Because the receiver and the transmitter are not synchronized to a master reference, there is bound to be a small frequency difference between the receiver and transmitter due to the accuracy of the equipment and thermal variation over time; consequently, the received signal cannot be down-converted properly. Although the magnitude of the frequency difference is small-usually smaller than $300 \mathrm{~Hz}$, compared to the carrier frequency, the signal quality can be degraded to the point that decoding becomes impossible. Hence, a Costas Loop is required to offset the small frequency difference before the signal is fed into the beam-former. The following section will not only discuss the problem with using the Costas Loop as a means for carrier recovery, but will also examine some potential solutions for avoiding this problem.

\section{A.1. Problem of Using a Digital Costas Loop to Recover the Carrier}

The input to the Costas Loop can be modeled by the following equation.

$$
s(t)=m_{r}(t) e^{j\left(m_{t}(t)\right)} e^{-j 2 \pi f_{r}\left(t_{t}+a \frac{p}{f_{t}} \cos \theta_{k}\right)} e^{j \omega_{r} t}
$$

where $m_{r}(t)$ is the amplitude part of the message, in the case of the QPSK $m_{r}(t)$ is 1 , and $m_{\theta}(t)$ is the phase part of the message. $e^{-j 2 \pi f_{1}\left(\tau_{t}+a \frac{p}{f_{1}} \operatorname{con} \theta_{t}\right.}$ 'is the phase shift because of the position of the antenna and the delay of the channel shown in Figure 4 and $e^{\prime w, r}$ is the 


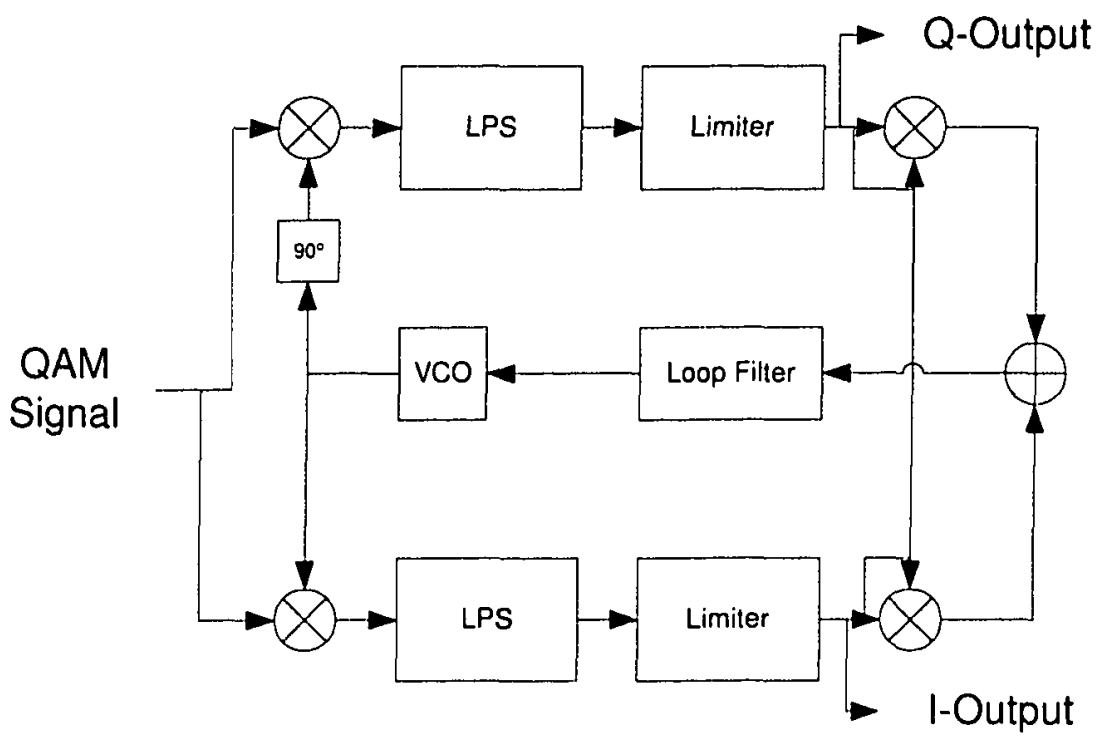

Figure 49. The diagram shows the structure of the Costas Loop for QAM4 signals.

residue carrier. The Costas Loop is able to remove the carrier by multiplying $s(t)$ with $e^{-j\left(\omega_{r} r+0\right)}$. (Figure 49 shows the structure of the Costas Loop.) At a steady state, the resulting signal is given by

$$
r(t)=s(t) e^{-j\left(\omega_{r} r+o\right)}=m_{r}(t) e^{j\left(m_{\theta}(t)\right)} e^{-j 2 \pi f_{1}\left(\tau_{i}+a \frac{\rho}{f_{i}} \cos \theta_{\lambda}\right)} e^{j o}
$$

The phase detector extracts the phase portion of the decoded signal and generates an error signal $e(t)$ for the voltage control oscillator (VCO), where

$$
e(t)=\omega t+m_{\theta}(t)+2 \pi f_{c}\left(\tau_{k}+a \frac{\rho}{f_{c}} \cos \theta_{k}\right)-\left(\omega_{r} t+\phi\right)
$$

The error signal $e(t)$ controls the oscillation frequency of the VCO, and when lock is achieved, $e(t)$ should be a DC signal. The operation of the VCO could be characterized by the following equation: 


$$
e^{-j((\alpha+\phi)}=e^{-j\left(\int e(t) d t\right)}
$$

Based on (57) and (58), the steady state condition can be described by the following equations:

$$
\begin{aligned}
& \omega t=\omega_{r} t \\
& e(t)=m_{\theta}(t)+2 \pi f_{c}\left(\tau_{k}+a \frac{\rho}{f_{c}} \cos \theta_{k}\right)-\phi
\end{aligned}
$$

Hence, the resulting signal will have a phase in which it is no longer dependent on the position of the antenna and the arrival angle of the signal. Instead, the phase of the incoming signal will depend solely on the frequency difference between the transmitter and the receiver.

If all the RF front ends in the receiver have the same frequency difference as the transmitter (this is possible when all the front end elements use the same clock source to down-covert the received signal), the phase information captured using the multiple antennas will be destroyed in the process. Hence, the performance of the beam-former to mitigate interferences is going to be severely reduced.

\section{A.2. Solutions for the Current System}

There are two RF front-end modules in the current system, both of which are connected to the same reference clock source: therefore. the current system setup would be susceptible to the problem described above. Although the RF front-end modules are assembled pre-built circuit boards, there are potentiometers on board, which allow the

user to tune the operating characteristics of the VCO. By tuning the potentiometers on 
the two front-end modules differently, the down-conversion frequency is different. In addition, the components used in each board have slightly different values; therefore, even if the boards are tuned to the same value, the response of the oscillator could still be different.

In case there is more than one signal path, the system could still maintain the relative phase difference of the two signal paths and therefore, it should not affect the interference mitigation ability of the beam-former. The incoming signal can be modeled as

$$
s_{0}(t)=\left(m_{r a}(t) e^{j\left(m_{\theta^{\prime}}(t)\right)}+m_{r b}(t) e^{j\left(m_{t+1}(t)\right)}\right) e^{j \omega_{r(0)} t}
$$

and

$$
s_{1}(t)=\left(m_{r l}(t) e^{j\left(m_{r t}(t)\right)}+m_{r b}(t) e^{j\left(m_{t,}(t)\right)}\right) e^{j \omega_{r 1} t} e^{-j 2 \pi f_{c}\left(\tau_{k}+a \frac{\rho}{f_{c}} \cos \theta_{k}\right)}
$$

where $s_{0}(t)$ is the signal received by antenna 0 and $s_{1}(t)$ is the signal received by antenna 1. After passing the signal through two different Costas Loops, the resulting signals become,

$$
r_{0}(t)=s_{0}(t) e^{j \phi_{0}}=\left(\left(m_{r a}(t) e^{j\left(m_{t a t}(t)\right)}+m_{r b}(t) e^{j\left(m_{r+p}(t)\right)}\right)\right) e^{j \phi_{0}}
$$

and

$$
\begin{aligned}
r_{i}(t) & =s_{1}(t) e^{j \phi_{1}} \\
& =\left(m_{r a}(t) e^{j\left(m_{\theta_{1}}(t)\right)}+m_{r b}(t) e^{j\left(m_{t a}(t)\right)}\right) e^{j \phi_{1}} e^{-j 2 \pi f_{t}\left(\pi_{t}+a \frac{\rho}{f_{t}} \cos \theta_{t}\right)}
\end{aligned}
$$

Consequently, the relative phases of each of the individual paths are preserved. 


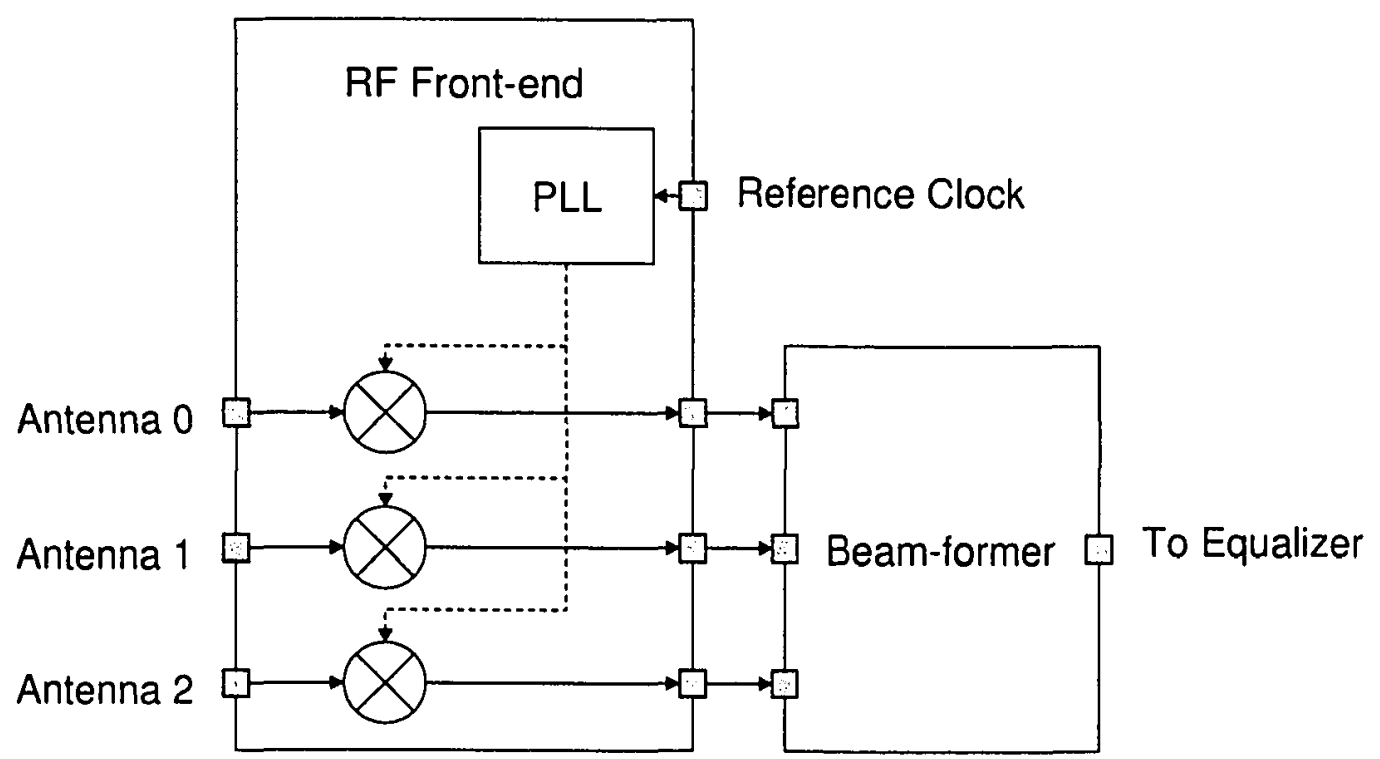

Figure 50. An alternate implementation of the capture system uses a single PLL to generate the IF frequency to down-convert the signal from the antenna.

\section{A.3. Solutions for Future Systems}

Because the RF front ends come pre-packaged, each front end has its own PLL to generate the required IF frequency. In the future, all the RF front ends should be integrated into a single module, as shown in Figure 50: therefore, only a single PLL would be required to down-convert from the IF frequency band. Because a single PLL is used, the transmitter and receiver frequency across all of the signals from each would be the same. Hence, it eliminates the problem of having to compensate for the carrier difference individually for each signal streams, and the relative phase difference can be preserved. 


\section{References}

[1] M.J Feuerstein, M.A. Zhao, Gu Yonghai, S.D. Gordon, "The future of smart antennas: evolution to $3 \mathrm{G}$ and IP networks," The I/th IEEE International Symposium on Personal, Indoor and Mobile Radio Communications 2000, vol 1, 18-21 Sept. 2000, pp. $48-54$.

12] L. C. Godara, "Application of Antenna Arrays to Mobile Communications, Part II: Beam-Forming and Direction-of-Arrival Considerations," Proceedings of the IEEE, vol. 85, no. 8, August 1997, pp.1195-1245.

[3] S. Haykin, Communication Systems, $3^{\text {rd }}$ ed., John Wiley \& Sons, 1994.

14] R. A. Monzingo, and T. W. Miller, Introduction to Adaptive Arrays, John Wiley \& Sons, Inc., 1980

[5] C. B. Dietrich, Jr., W. L. Stutzman, B. Kim, and K. Dietze, "Smart Antennas in Wireless Communications: Base-Station Diversity and Handset Beam-forming”. IEEE Antennas and Propagation Magazine, Vol. 32, no. 5, October 2000, pp. $142-151$

(6) I. Chiba, R. Miura, T. Tanaka, Y. Karasawa. "Digital Beam Forming Antenna System for Mobile Communication," IEEE Aerospace and Electronic Systems Magazine, vol. 12, no. 9, Sept. 1997, pp. $31-41$.

17] T. Miyajima, "Blind Adaptive Detection Using Differential CMAs for CDMA Systems", IEICE Transaction Communication, Vol. J83-A, No.11, November 2000, pp.1318-1329. 
18] R. Martínez, A. Cacho, L. Haro, M. Calvo, "Comparative study of LMS and RLS adaptive algorithms in the optimum combining of uplink W-CDMA", Proceedings of IEEE 56th Vehicular Technology Conference, vol. 4, 24-28 September 2002, pp. $2258-2262$.

[9] Y. C. Liang, F. P.S. Chin, "Coherent LMS algorithms", IEEE Communications Letters, vol. 4, no. 3, March 2000, pp. $92-94$.

[10] Alexander S. Thomas, Adaptive Signal Processing: Theory and Applications, Springer-Verlag, New York, 1986.

[II] J. C. L. Ng, K. B. Letaief and R. D. Murch, "Antenna Diversity Combining and Finite-Tap decision Feedback Equalization for High-Speed Data Transmission," IEEE Journals on Selected Areas in Communications, vol. 16, no. 8, Oct. 1998. pp. $1367-1375$.

112] J. F. Frigon, B. Daneshrad, "Field Measurements of an Indoor High-Speed QAM Wireless System Using Decision Feedback Equalization and Smart Antenna Array", IEEE Transactions on Wireless Communications, vol. 1, no 1. January 2002, pp. $134-144$.

[13] H. Matsuoka, Y. Murakami, H. Shoki, and Y. Suzuki, "A Smart Antenna Receiver Testbed with Directional Antenna Elements", Proceedings of IEEE International Conference on Phased Array Systems and Technology, 21-25 May 2000, pp. $113-116$ 
[14] H. J. Im and S. Choi," Performance Analysis of Smart Antenna Test-Bed Operating in a Wide-Band CDMA Channel", IEEE Transactions: On Microwave Theory And Techniques, vol. 49, no. 11, November 2001, pp. $2142-2146$.

[15] C. R. Johnson, Jr., H.J. Lee, J.P. LeBlanc, T.J. Endres, R.A. Casas, E. Tai, Z. Reznic, W.E. Meyer, F. Lopez de Victoria, J.R. Treichler, I. Figalkow, and Z. Ding, "On Fractionally-Spaced Equalizer Design for Digital Microwave Radio Channels", Conference Record of the Twenty-Ninth Asilomar Conference on Signals, Systems and Computers 1995, vol. 1, 30 Oct.-2 Nov. 1995, pp. 290 294.

[16] H. Meyr, M. Moeneclaey, and S. A. Fechtel, Digital communication receivers: synchronization, channel estimation, and signal processing, Wiley, New York. 1998.

117] C. Dick, M. Rice, F. Harris, "Configurable logic for wireless communications: carrier and symbol synchronization", Proceedings of Imternational IC Conference - Taipei, 3 May 2003, pp. 138 - 151.

|181 Z. Sheng, W. Weiling. "Performance Analysis of Pilot Signal Aided Coherent Rake Receiver in IMT-2000 System", Proceedings of International Conference on Communication Technology 2000, vol. 1, 21 -25 Aug. 2000, pp. 733 - 736.

[19] M. T. Ma, Theory and Application of Antemna Arrays, John Wiley \& Sons, Inc., 1974 
[20] L. Jie, Z. Jian-wu, "The Adaptive Algorithms of the Smart Antenna System in 3G Wireless Communication Systems," 6th International Conference on Signal Processing 2002, vol. 2, August 2002, pp. $1664-1667$.

[21] A. U. Bhobe, P. L. Perini, "An Overview of Smart Antenna Technology for Wireless Communication", IEEE Proceedings Aerospace Conference 200l, vol. 2, 10-17 March 2001, pp $875-883$.

[22] R. Martinez, A. del Cacho, L. de Haro, and M. Calvo, "Comparative study of LMS and RLS adaptive algorithms in the optimum combining of uplink WCDMA," IEEE 56th Vehicular Technology Conference 2002, vol. 4, Sept. 2002, pp. $2258-2262$.

[23] K. Phillips, Z. Hu, K. Blankenship, Z. Siddiqi, N. Correal, "Implementation of an Adaptive Antenna Array Using the TMS320C541" (SPRA532)

[24] W. F. Gabriel, "Adaptive Processing Array System", Proceedings of the IEEE, Volume 80, No. 1, Jan. 1992, pp. 152-162.

[25] R. D. Murch and K. B. Letaief, "Antenna Systems for Broadband Wireless Access", IEEE Communications Magazine, Vol. 40, no. 4. April 2002. pp. 76 83.

[26] G. German, Q. Spencer, A. Swindlehurst, and R. Valenzuela, "Wireless Indoor Channel Modeling: Statistical Agreement of Ray Tracing Simulations and Channel Sounding Measurements", Proceeding of IEEE International Conference on Acoustics, Speech, and Signal Processing. 2001, vol. 4. 7-11 May 2001, pp. $2501-2504$. 
[27] C. Y. Chi, and C. Y. Chen, "Blind Beamforming and Maximum Ratio Combining by Kurtosis Maximization for Source Separation in Multipath", Third IEEE Signal Processing Workshop on Signal Processing Advances in Wireless Communications 2001, 20-23 March, 2001, pp. 243-246.

281 A. H. El Zooghby, C. G. Christodoulou, and M. Georgiopoulos, "A Neural Network-Based Smart Antenna for Multiple Source Tracking", IEEE Transactions on Antemas And Propagation, vol. 48, no. 5, May 2000, pp. 768 776.

[29] M. Godavarti, Multiple Antennas in Wireless Communications: Array Signal Processing and Channel Capacity, Thesis, University of Michigan, 2001.

[30] S. S. Jeon, Y. Wan, Y. Qian, T. Itoh, "A Novel Smart Antenna System Implementation for Broad-Band Wireless Communications", IEEE Transactions on Antennas and Propagation, vol. 50, no, 5, May 2002, pp. $600-606$.

[31] M. Piasecki, J. Modelski, "Investigation of an Experimental Setup of a Smart Antenna," IEEE MTT-S International Microwave Symposium Digest 2003, vol. $3,8-13$ June 2003, pp. $1845-1848$.

[32] T. Le-Ngoc, and A. Haghighat, "A Low-jitter Symbol Timing Recovery Scheme for M-QAM Systems", Proceedings of IEEE 1997 Canadian Conference on Electrical and Computer Engineering, vol. 1, 25-28 May 1997, pp. 201 - 204.

[33] T. Eireiner, T. Muller, J. F. Luy, F. Owens, "Implementation of a smart antenna system with an improved NCMA algorithm", IEEE MTT-S International Microwave Symposilum Digest, vol. 3, 8-13 June 2003, pp. 1529 - 1532. 
[34] D. V. Compernolle, and S. V. Gerven, "Signal Separation in A Symmetric Adaptive Noise Canceller by Output Decorrelation", Proceedings of IEEE International Conference on Acoustics, Speech, and Signal Processing 1992, vol 4., 23-26 March 1992, pp. 221 - 224.

[35] T. Chulajata, and H. M. Kwon, "An adaptive array antenna with no phase calibration for cdma2000 reverse link", Proceedings of 2 Ist Century Military Communications Conference, vol. 2, 22-25 Oct. 2000, pp. 816-820.

[36] A. T. Bu, X. W. Shi, Y. Liu, L. Y. Xiao, “”Design of Sector Array Antenna Based on Genetic Algorithm for Smart Antenna System Front End," IEEE Society International Conference on Antennas and Propagation 2003, vol. 3, 2227 June 2003, pp. $686-689$.

[37] Y. Takatori, K. Cho, K. Nishimori, and T. Hori, "Adaptive Array Employing Eigenvector Beam of Maximum Eigenvalue and Fractionally-Spaced TDL with Real Tap”, IEICE Transaction Communication. vol. E83-B, no.8, August 2000, pp. $1678-1686$.

[38] J. H. Winters, "Smart Antennas for Wireless Systems", IEEE Personal Communications, vol. 5, no. I. February 1998, pp. $23-27$. 